H. Tamura

Nagoya Math. J.

Vol. 155 (1999), 95-151

\title{
MAGNETIC SCATTERING AT LOW ENERGY IN TWO DIMENSIONS
}

\author{
HIDEO TAMURA
}

\author{
Dedicated to Professor Kiyoshi Mochizuki on his 60th birthday
}

\begin{abstract}
We study the asymptotic behavior at low energy of scattering amplitudes in two dimensional magnetic fields with compact support. The obtained result depends on the total flux of magnetic fields. It should be noted that magnetic potentials do not necessarily fall off rapidly at infinity. The main body of argument is occupied by the resolvent analysis at low energy for magnetic Schrödinger operators with perturbations of lang-range class. We can show that the dimension of resonance spaces at zero energy does not exceed two. As a simple application, we also discuss the scattering by magnetic field with small support and the convergence to the scattering amplitude by $\delta$-like magnetic field.
\end{abstract}

\section{$\S 1$. Introduction}

In the present work we consider the low-energy scattering for Schrödinger operators with magnetic fields compactly supported in two dimensions. We study the asymptotic behavior at low energy of scattering amplitudes. As a direct application, we also discuss the behavior of scattering amplitudes for scattering by magnetic fields with small support. The results obtained strongly depend on the total flux of magnetic fields under consideration.

Throughout the entire discussion, we work in the two dimensional space $\mathbf{R}^{2}$ with generic point $x=\left(x_{1}, x_{2}\right)$. Let $b(x): \mathbf{R}^{2} \rightarrow \mathbf{R}$ be a given magnetic field. We assume that $b \in C_{0}^{\infty}\left(\mathbf{R}^{2}\right)$ is a smooth function with compact support, and we denote by

$$
\alpha=(2 \pi)^{-1} \int b(x) d x
$$

the total flux of field $b$, where the integration with no domain attached is taken over the whole space. This abbreviation is used throughout. We

Received May 11, 1998. 
also assume that $0<\alpha<1$. The argument below extends with a natural modification to the case that $\alpha \notin \mathbf{Z}$ is not an integer. We now define

$$
A_{\alpha}(x)=\left(a_{1 \alpha}(x), a_{2 \alpha}(x)\right)=\left(-\partial_{2} \varphi(x), \partial_{1} \varphi(x)\right), \quad \partial_{j}=\partial / \partial x_{j}
$$

where $\varphi(x)=(2 \pi)^{-1} \int \log |x-y| b(y) d y$. Then we have $\nabla \times A_{\alpha}=\partial_{1} a_{2 \alpha}-$ $\partial_{2} a_{1 \alpha}=\Delta \varphi=b$ and hence $A_{\alpha}$ becomes the magnetic potential associated with field $b(x)$. As is easily seen, $A_{\alpha}(x)$ behaves like

$$
A_{\alpha}(x)=B_{\alpha}(x)+O\left(|x|^{-2}\right), \quad B_{\alpha}(x)=\alpha\left(-x_{2} /|x|^{2}, x_{1} /|x|^{2}\right)
$$

as $|x| \rightarrow \infty$. The magnetic potential $A(x): \mathbf{R}^{2} \rightarrow \mathbf{R}^{2}$ associated with field $b(x)$ is not uniquely determined, but the scattering operator is invariant under the gauge transformation $A \rightarrow A+\nabla g$. Thus we may fix one of such magnetic potentials, which behaves like (1.2) at infinity. It should be noted that magnetic potentials never decays faster than $O\left(|x|^{-1}\right)$ at infinity, even if $b(x)$ is assumed to be of compact support. In section 2 , we will specify the more precise form of magnetic potential $A(x)$, which coincides with $B_{\alpha}(x)$ for $|x| \gg 1$.

Let $A(x)=\left(a_{1}(x), a_{2}(x)\right)$ be as above and let $V \in C_{0}^{\infty}\left(\mathbf{R}^{2}\right): \mathbf{R}^{2} \rightarrow \mathbf{R}$ be a given electric potential. We consider the Hamiltonian

$$
H=H(A, V)=(-i \nabla-A)^{2}+V=\sum_{j=1}^{2}\left(-i \partial_{j}-a_{j}\right)^{2}+V .
$$

The operator $H$ formally defined above has a unique self-adjoint realization in $L^{2}\left(\mathbf{R}^{2}\right)$. We denote by the same notation $H$ this realization with domain $\mathcal{D}(H)=H^{2}\left(\mathbf{R}^{2}\right)$ (Sobolev space). By (1.2), the difference $H-H_{0}$ between $H$ and the free Hamiltonian $H_{0}=-\Delta$ belongs to the long-range perturbation class. Nevertheless we know $([8],[10])$ that the ordinary wave operators

$$
W_{ \pm}\left(H, H_{0}\right)=s-\lim _{t \rightarrow \pm \infty} \exp (i t H) \exp \left(-i t H_{0}\right): L^{2}\left(\mathbf{R}^{2}\right) \longrightarrow L^{2}\left(\mathbf{R}^{2}\right)
$$

exist and are asymptotically complete

$$
\operatorname{Ran}\left(W_{-}\left(H, H_{0}\right)\right)=\operatorname{Ran}\left(W_{+}\left(H, H_{0}\right)\right) .
$$

Hence the scattering matrix $S\left(\lambda ; H, H_{0}\right): L^{2}\left(S^{1}\right) \rightarrow L^{2}\left(S^{1}\right)$ at energy $\lambda>0, S^{1}$ being the unit circle, can be defined as a unitary operator. Let 
$S\left(\omega^{\prime}, \omega ; \lambda\right),\left(\omega^{\prime}, \omega\right) \in S^{1} \times S^{1}$, denote the integral kernel of $S\left(\lambda ; H, H_{0}\right)$. Then the scattering amplitude $f\left(\omega \rightarrow \omega^{\prime} ; \lambda\right)$ for scattering from incident direction $\omega$ into final one $\omega^{\prime}$ at energy $\lambda>0$ is defined by

$$
f\left(\omega \rightarrow \omega^{\prime} ; \lambda\right)=c(\lambda)\left(S\left(\omega^{\prime}, \omega ; \lambda\right)-\delta\left(w^{\prime}-\omega\right)\right)
$$

with $c(\lambda)=(2 \pi / i \sqrt{\lambda})^{1 / 2}$. The precise representation for $f\left(\omega \rightarrow \omega^{\prime} ; \lambda\right)$ is given in section 3 (see Proposition 3.1). It is represented through the resolvent

$$
R(\lambda+i 0 ; H)=\lim _{\varepsilon \rightarrow 0} R(\lambda+i \varepsilon ; H), \quad R(z)=(H-z)^{-1} .
$$

The first aim of the present work is to study the behavior as $\lambda \rightarrow 0$ of scattering amplitude $f\left(\omega \rightarrow \omega^{\prime} ; \lambda\right)$. The main theorem is formulated as Theorem 7.1 in section 7 . We here mention it somewhat loosely. The behavior depends on the resonance space $\mathcal{E}_{1}$ at zero energy of $H=H(A, V)$. Roughly speaking, $\mathcal{E}_{1}$ is defined as

$$
\mathcal{E}_{1}=\left\{u \in L_{\text {loc }}^{2}\left(\mathbf{R}^{2}\right): u(x) \text { is bounded, } H u=0\right\} / \mathcal{E}_{0}
$$

with the zero eigenspace $\mathcal{E}_{0}=\left\{u \in L^{2}\left(\mathbf{R}^{2}\right): H u=0\right\}$. If the flux $\alpha$ of field $b$ is not an integer, then it is shown that $\operatorname{dim} \mathcal{E}_{1} \leq 2$. If $\operatorname{dim} \mathcal{E}_{1}=0$, then we can prove that

$$
f\left(\omega \rightarrow \omega^{\prime} ; \lambda\right)=c(\lambda)\left(f_{\alpha}\left(\omega^{\prime}-\omega ; \lambda\right)+o(1)\right), \quad \lambda \rightarrow 0
$$

where

$$
f_{\alpha}\left(\omega^{\prime}-\omega\right)=(\cos \alpha \pi-1) \delta\left(\omega^{\prime}-\omega\right)-(i \sin \alpha \pi / \pi) F_{0}\left(\omega^{\prime}-\omega\right)
$$

with $F_{0}(\theta)=$ v.p. $e^{i \theta} /\left(e^{i \theta}-1\right)$, and the coordinates over $S^{1}$ are identified with the azimuth angles from the positive $x_{1}$ axis. The leading term just coincides with the scattering amplitude calculated by [1] (see [11] also) for the Hamiltonian

$$
H_{\alpha}=\left(-i \nabla-B_{\alpha}\right)^{2}
$$

with domain

$$
\mathcal{D}\left(H_{\alpha}\right)=\left\{u \in L^{2}\left(\mathbf{R}^{2}\right): H_{\alpha} u \in L^{2}\left(\mathbf{R}^{2}\right), \lim _{|x| \rightarrow 0} u=0\right\},
$$

where $H_{\alpha} u=\left(-i \nabla-B_{\alpha}\right)^{2} u$ is understood in $\mathcal{D}^{\prime}$ (in the distributional sense). If, in particular, $V(x)=0$, then we can show that $\operatorname{dim} \mathcal{E}_{1}=0$ and 
the above asymptotic formula is obtained. When $\operatorname{dim} \mathcal{E}_{1}=2, \mathcal{E}_{1}$ is spanned by a pair $\left(\rho_{0}, \rho_{1}\right)$ of functions taking the form

$$
\rho_{l}(x)=r^{-\nu} e^{i l \theta}+g_{l}, \quad \nu=|l-\alpha|,
$$

with some $g_{l} \in L^{2}\left(\mathbf{R}^{2}\right)$, where $(r, \theta)$ denotes the polar coordinates over $\mathbf{R}^{2}$, and $f\left(\omega \rightarrow \omega^{\prime} ; \lambda\right)$ is shown to behave like

$$
f\left(\omega \rightarrow \omega^{\prime} ; \lambda\right)=c(\lambda)\left(f_{\alpha}\left(\omega^{\prime}-\omega\right)+(i / \pi) \sin \alpha \pi\left(1-e^{i\left(\omega^{\prime}-\omega\right)}\right)+o(1)\right) .
$$

On the other hand, if $\operatorname{dim} \mathcal{E}_{1}=1$, then $\mathcal{E}_{1}$ is spanned by a linear combination

$$
\rho(x)=c_{0} r^{-\alpha}+c_{1} r^{-(1-\alpha)} e^{i \theta}+g, \quad g \in L^{2}\left(\mathbf{R}^{2}\right),
$$

and the asymptotic formula as $\lambda \rightarrow 0$ of $f\left(\omega \rightarrow \omega^{\prime} ; \lambda\right)$ takes various forms according to the value $\alpha$ and the ratio $c=c_{1} / c_{0}$.

In section 8 , we discuss the scattering by magnetic fields with small support as a simple application of the low-energy scattering. Let $b(x), V(x) \in$ $C_{0}^{\infty}\left(\mathbf{R}^{2}\right)$ and $A(x), \nabla \times A=b$, be as above. We set

$$
b_{\varepsilon}(x)=\varepsilon^{-2} b(x / \varepsilon), \quad V_{\varepsilon}(x)=\varepsilon^{-2} V(x / \varepsilon), \quad A_{\varepsilon}(x)=\varepsilon^{-1} A(x / \varepsilon)
$$

for $0<\varepsilon \ll 1$, and we consider the Hamiltonian

$$
H_{\varepsilon}=H\left(A_{\varepsilon}, V_{\varepsilon}\right)=\left(-i \nabla-A_{\varepsilon}\right)^{2}+V_{\varepsilon}
$$

As is easily seen, $\nabla \times A_{\varepsilon}=b_{\varepsilon}$ and the field $b_{\varepsilon}$ preserves the flux

$$
(2 \pi)^{-1} \int b_{\varepsilon}(x) d x=\alpha .
$$

We further have $A_{\varepsilon}(x) \rightarrow B_{\alpha}(x)$ and $b_{\varepsilon}(x) \rightarrow 2 \pi \alpha \delta(x)$ in $\mathcal{D}^{\prime}$ as $\varepsilon \rightarrow 0$. Thus $H_{\varepsilon}$ is formally convergent to the Hamiltonian

$$
H_{0 \alpha}=\left(-i \nabla-B_{\alpha}\right)^{2}, \quad \mathcal{D}\left(H_{0 \alpha}\right)=C_{0}^{\infty}\left(\mathbf{R}^{2} \backslash\{0\}\right)
$$

with $\delta$-like magnetic field at the origin. Let $f_{\varepsilon}\left(\omega \rightarrow \omega^{\prime} ; \lambda\right)$ be the scattering amplitude for the pair $\left(H_{\varepsilon}, H_{0}\right)$. The second aim is to analyse the behavior as $\varepsilon \rightarrow 0$ of $f_{\varepsilon}\left(\omega \rightarrow \omega^{\prime} ; \lambda\right)$ for energy $\lambda>0$ fixed. By making a change $x / \varepsilon \rightarrow x$ of variables, $f_{\varepsilon}\left(\omega \rightarrow \omega^{\prime} ; \lambda\right)$ can be easily shown to satisfy the relation

$$
f_{\varepsilon}\left(\omega \rightarrow \omega^{\prime} ; \lambda\right)=\sqrt{\varepsilon} f\left(\omega \rightarrow \omega^{\prime} ; \lambda \varepsilon^{2}\right)
$$


and hence the problem is reduced to the study on the asymptotic behavior of the scattering amplitude $f\left(\omega \rightarrow \omega^{\prime} ; \lambda \varepsilon^{2}\right)$ for the pair $\left(H, H_{0}\right), H=H(A, V)$, at low energy $\lambda \varepsilon^{2}$. This problem is motivated by the recent work [2], where the self-adjoint extension of $H_{0 \alpha}$ is discussed in detail and the scattering amplitude for such a self-adjoint operator is calculated (see [14] also). According to the result there, the operator $H_{0 \alpha}$ has the deficiency indices $(2,2)$, and there exists a family of self-adjoint extensions parameterized by $2 \times 2$ unitary mapping $U$ from one deficiency subspace to the other one. The operator $H_{\alpha}$ defined by (1.3) is one of such self-adjoint extensions. The unitary mapping specifies the boundary condition at the origin for the domain of self-adjoint extensions. We are concerned with the boundary condition realized in the limit $\varepsilon \rightarrow 0$. The result obtained again strongly depends on the structure of the resonance space $\mathcal{E}_{1}$ at zero energy of $H=H(A, V)$. The precise result is formulated as Theorems 8.1 and 8.2 in section 8 .

A lot of works ([3], [4], [7], [9], [12]) have been already done on the behavior at low energy of scattering amplitudes and on the approximation to point interactions in the case of short-range potential scattering. An extensive list of related literatures can be found in the book [3]. We basically follow the idea developed in Jensen-Kato [7], althogh several technical improvements are required at many stages of the proof. The standard way to analyse the behavior of resolvents at low energy is based on the relation

$$
R(\lambda+i 0 ; H)=\left(\mathrm{Id}+R\left(\lambda+i 0 ; H_{0}\right)\left(H-H_{0}\right)\right)^{-1} R\left(\lambda+i 0 ; H_{0}\right)
$$

obtained from the resolvent identity, where Id stands for the identity operator. For the case of scattering by magnetic fields, the difference $H-H_{0}$ is not necessarily of short-range class even for the field $b(x)$ compactly supported, as previously stated. The resolvent identity does not work for the pair $\left(H, H_{0}\right)$. On the other hand, $H-H_{\alpha}$ becomes a perturbation of shortrange class for the Hamiltonian $H_{\alpha}$ defined by (1.3), but the domain $\mathcal{D}\left(H_{\alpha}\right)$ does not coincide with that of $H$. It should be noted that even the form domains of these operators are different. This makes it difficult to use the resolvent identity for the pair $\left(H, H_{\alpha}\right)$ also. Thus we take a slightly different approach. We introduce a certain auxiliary operator $K_{\alpha}=\left(-i \nabla-\chi_{\infty} B_{\alpha}\right)^{2}$, where $\chi_{\infty}(r), r=|x|$, is a smooth real function vanishing near the origin and taking the value $\chi_{\infty}=1$ for $r \gg 1$ large enough. By definition, $K_{\alpha}$ has the same domain as $H$, and the difference $W=H-K_{\alpha}$ belongs to the 
short-range class. In addition, $K_{\alpha}$ admits the partial wave expansion in angular momentum. This enables us to expand $R\left(\lambda+i 0 ; K_{\alpha}\right)$ asymptotically in $\lambda, 0<\lambda \ll 1$, small enough and to analyse the behavior at low energy of resolvent $R(\lambda+i 0 ; H)$ in question through relation (1.5) applied to the pair $\left(H, K_{\alpha}\right)$. The main body of the present work is occupied by the low-energy analysis for resolvents of magnetic Schrödinger operators with long-range perturbations. Such an analysis is also important in showing the resolvent convergence in norm of the scaled Hamiltonian $H_{\varepsilon}$ to some self-adjoint extension of $H_{0 \alpha}$. The matter will be discussed in detail elsewhere.

\section{$\S 2$. Magnetic Schrödinger operator}

In this section we fix the magnetic Schrödinger operator $H=H(A, V)$ to be analysed throughout the entire discussion. We always assume that $b, V \in C_{0}^{\infty}\left(\mathbf{R}^{2}\right): \mathbf{R}^{2} \rightarrow \mathbf{R}$ are real smooth functions with support in the unit ball

$$
\operatorname{supp} b, \operatorname{supp} V \subset\left\{x \in \mathbf{R}^{2}:|x|<1\right\} .
$$

We further assume that the total flux $\alpha, \alpha \notin \mathbf{Z}$ not being an integer, of the field $b(x)$ satisfies $0<\alpha<1$. The magnetic potential $A_{\alpha}(x)=$ $\left(a_{1 \alpha}(x), a_{2 \alpha}(x)\right)$ defined by $(1.1)$ is easily shown to have the following properties.

Lemma 2.1. Let $B_{\alpha}(x)=\alpha\left(-x_{2} /|x|^{2}, x_{1} /|x|^{2}\right)$ be again as in (1.2). Then one has:

(1) $A_{\alpha}(x)$ is smooth and obeys $\partial_{x}^{\beta} A_{\alpha}(x)=O\left(|x|^{-1-|\beta|}\right)$ as $|x| \rightarrow \infty$.

(2) $A_{\alpha}(x)$ behaves like

$$
\partial_{x}^{\beta} A_{\alpha}(x)=\partial_{x}^{\beta} B_{\alpha}(x)+O\left(|x|^{-2-|\beta|}\right), \quad|x| \rightarrow \infty,
$$

and, in particular,

$$
\partial_{x}^{\beta}\left(x_{1} a_{1 \alpha}(x)+x_{2} a_{2 \alpha}(x)\right)=O\left(|x|^{-1-|\beta|}\right) .
$$

The lemma above enables us to define $a_{\alpha}(x)$ as

$$
a_{\alpha}(x)=-\int_{1}^{\infty}\left(x_{1} a_{1 \alpha}(s x)+x_{2} a_{2 \alpha}(s x)\right) d s .
$$

Lemma 2.2. Let $a_{\alpha}(x)$ be as above. Then one has:

(1) $a_{\alpha}(x)$ is smooth in $R^{2} \backslash\{0\}$, and it obeys the bound

$$
\partial_{x}^{\beta} a_{\alpha}(x)=O\left(|x|^{-1-|\beta|}\right), \quad|x| \rightarrow \infty .
$$


(2) $A_{\alpha}(x)$ is represented as

$$
A_{\alpha}(x)=B_{\alpha}(x)+\nabla a_{\alpha}(x)+E_{\alpha}(x), \quad x \neq 0,
$$

where $E_{\alpha}(x)=\left(e_{1}(x), e_{2}(x)\right)$ is given by

$$
e_{1}(x)=\int_{1}^{\infty} s x_{2} b(s x) d s, \quad e_{2}(x)=-\int_{1}^{\infty} s x_{1} b(s x) d s
$$

and has support in the unit ball.

Proof. (1) This follows from Lemma 2.1 at once. (2) To prove this, we set

$$
b_{j k}(x)=\partial_{j} a_{k \alpha}(x)-\partial_{k} a_{j \alpha}(x), \quad 1 \leq k, j \leq 2,
$$

so that $b(x)=b_{12}(x)=-b_{21}(x)$. A simple calculation yields

$$
\partial_{j} a_{\alpha}(x)=-\int_{1}^{\infty}\left(a_{j \alpha}(s x)+s(d / d s) a_{j \alpha}(s x)+s x_{k} b_{j k}(s x)\right) d s
$$

for $k \neq j$ and hence we obtain

$$
\partial_{j} a_{\alpha}(x)=a_{j \alpha}(x)-\int_{1}^{\infty} s x_{k} b_{j k}(s x) d s-\lim _{R \rightarrow \infty} R a_{j \alpha}(R x)
$$

by partial integration. By Lemma 2.1 again, $R A_{\alpha}(R x) \rightarrow B_{\alpha}(x)$ as $R \rightarrow \infty$. This proves (2) and the proof of the lemma is completed.

We here introduce a basic cut-off function $\chi \in C_{0}^{\infty}([0, \infty))$ such that $\chi(s)$ is nonnegative and

$$
\chi(s)=1 \quad \text { for } 0 \leq s \leq 1, \quad \chi(s)=0 \quad \text { for } s>2 .
$$

We set $\chi_{0}(x)=\chi_{0}(r)=\chi(r), r=|x|$, and $\chi_{\infty}(x)=1-\chi(r)$. Then $\chi_{\infty} E_{\alpha}=0$ for $E_{\alpha}(x)$ in Lemma 2.2, and hence $A_{\alpha}(x)$ is decomposed into

$$
A_{\alpha}=\left(\chi_{\infty}+\chi_{0}\right) A_{\alpha}=A(x)+\nabla\left(\chi_{\infty} a_{\alpha}\right)
$$

by Lemma 2.2, where

$$
A(x)=\chi_{\infty}(x) B_{\alpha}(x)+B(x)
$$

with $B=a_{\alpha} \nabla \chi_{0}+\chi_{0} A_{\alpha}$. By definition, $A(x)$ has still the magnetic field $b(x)$ and satisfies

$$
A(x)=B_{\alpha}(x)
$$


for $|x|>2$. We now fix

$$
H=H(A, V)=(-i \nabla-A)^{2}+V
$$

as the Hamiltonian with magnetic field $b(x)$ and electric potential $V(x)$, and we denote by the same notation $H$ the self-adjoint realization in $L^{2}\left(\mathbf{R}^{2}\right)$. The scattering operator is invariant under the gauge transformation $A \rightarrow$ $A+\nabla g$, provided that $g(x)$ falls off at infinity. The above magnetic potential $A(x)$ is written as $A=A_{\alpha}+\nabla g$ with $g=-\chi_{\infty} a_{\alpha}$, and $g(x) \rightarrow 0$ at infinity by Lemma 2.2. Hence both the pairs $\left(H(A, V), H_{0}\right)$ and $\left(H\left(A_{\alpha}, V\right), H_{0}\right)$ have the same scattering operator. The remaining sections are devoted to the analysis on the behavior at low energy of the scattering amplitude for the pair $\left(H, H_{0}\right)$.

\section{$\S 3$. Scattering amplitude}

The aim here is to represent the scattering amplitude for the pair $\left(H, H_{0}\right), H_{0}=-\Delta$, with $H=H(A, V)$ defined as above. This is done in a rather formal way. The rigorous justification can be found in [13].

Let $\varphi_{0}(x ; \lambda, \omega)=\exp (i \sqrt{\lambda} x \cdot \omega), \lambda>0, \omega \in S^{1}$, be the generalized eigenfunction of the free Hamiltonian $H_{0}, H_{0} \varphi_{0}=\lambda \varphi_{0}$, where the notation - denotes the scalar product in $\mathbf{R}^{2}$. As is well known, $\varphi_{0}$ is expanded as

$$
\varphi_{0}(x ; \lambda, \omega)=\sum_{l \in \mathbf{Z}} \exp (i|l| \pi / 2) \exp (i l \theta(x ; \omega)) J_{|l|}(\sqrt{\lambda}|x|)
$$

in terms of the Bessel functions $J_{p}(z)$, where $\theta(x ; \omega)$ is the azimuth angle from direction $\omega \in S^{1}$. Let $\mathcal{F}_{0}: L^{2}\left(\mathbf{R}^{2}\right) \rightarrow L^{2}((0, \infty) ; d \lambda) \otimes L^{2}\left(S^{1}\right)$ be the unitary mapping defined by

$$
\mathcal{F}_{0} u(\lambda, \omega)=2^{-1 / 2}(2 \pi)^{-1} \int \bar{\varphi}_{0}(x ; \lambda, \omega) u(x) d x .
$$

Then $H_{0}$ is diagonalized in such a way that

$$
\mathcal{F}_{0}^{*} H_{0} \mathcal{F}_{0}=\lambda \times \quad \text { on } L^{2}((0, \infty) ; d \lambda) \otimes L^{2}\left(S^{1}\right) .
$$

We work in the polar coordinates $(r, \theta)$ over $\mathbf{R}^{2}$ and write $L^{2}\left(\mathbf{R}_{+}\right)$for $L^{2}((0, \infty) ; d r)$. If we denote by $\Lambda_{l}, l \in \mathbf{Z}$, the eigenspace of operator $-i \partial / \partial \theta$ acting on $L^{2}\left(S^{1}\right)$ with eigenvalue $l$, then we have the decomposition

$$
L^{2}\left(\mathbf{R}_{+}\right) \otimes L^{2}\left(S^{1}\right)=\sum_{l \in \mathbf{Z}} \oplus\left(L^{2}\left(\mathbf{R}_{+}\right) \otimes \Lambda_{l}\right) .
$$


We further define the unitary mapping

$$
(U u)(r, \theta)=r^{1 / 2} u(r \theta): L^{2}\left(R^{2}\right) \longrightarrow L^{2}\left(\mathbf{R}_{+}\right) \otimes L^{2}\left(S^{1}\right) .
$$

Let $B_{\alpha}(x)=\alpha\left(-x_{2} /|x|^{2}, x_{1} /|x|^{2}\right)$ be as in (1.2) and let $H_{\alpha}=\left(-i \nabla-B_{\alpha}\right)^{2}$ be again defined by (1.3) with domain

$$
\mathcal{D}\left(H_{\alpha}\right)=\left\{u \in L^{2}\left(\mathbf{R}^{2}\right): H_{\alpha} u \in L^{2}\left(\mathbf{R}^{2}\right), \lim _{r \rightarrow 0} u=0\right\} .
$$

This operator is rotationally invariant and the unitary mapping $U$ yields the partial wave expansion. We formally write its expansion as

$$
H_{\alpha} \simeq U H_{\alpha} U^{*}=\sum_{l \in \mathbf{Z}} \oplus\left(H_{l \alpha} \otimes \mathrm{Id}\right),
$$

where $H_{l \alpha}$ is given by

$$
H_{l \alpha}=\Pi^{*} \Pi+\nu^{2} r^{-2}=-\partial_{r}^{2}+\left(\nu^{2}-1 / 4\right) r^{-2}, \quad \Pi=\partial_{r}-1 / 2 r,
$$

with $\nu=|l-\alpha|$. The operator $H_{l \alpha}$ is self-adjoint in $L^{2}\left(\mathbf{R}_{+}\right)$with domain

$$
\mathcal{D}\left(H_{l \alpha}\right)=\left\{u \in L^{2}\left(\mathbf{R}_{+}\right): H_{l \alpha} u \in L^{2}\left(\mathbf{R}_{+}\right), \lim _{r \rightarrow 0} r^{-1 / 2} u(r)=0\right\}
$$

and hence $H_{\alpha}$ also becomes self-adjoint in $L^{2}\left(\mathbf{R}^{2}\right)$ with domain $\mathcal{D}\left(H_{\alpha}\right)$ as above. Since the magnetic potential $B_{\alpha}(x)$ has a strong singularity at the origin, $\mathcal{D}\left(H_{\alpha}\right)$ does not necessarily coincide with the domain of operators $H$ and $H_{0}$. Nevertheless it is known ([11]) that the wave operators

$$
W_{ \pm}\left(H_{\alpha}, H_{0}\right)=s-\lim _{t \rightarrow \pm \infty} \exp \left(i t H_{\alpha}\right) \exp \left(-i t H_{0}\right): L^{2}\left(\mathbf{R}^{2}\right) \longrightarrow L^{2}\left(\mathbf{R}^{2}\right)
$$

exist and are asymptotically complete $\operatorname{Ran} W_{ \pm}\left(H_{\alpha}, H_{0}\right)=L^{2}\left(\mathbf{R}^{2}\right)$.

The generalized eigenfunction $\varphi_{\mp}(x ; \lambda, \omega)$ of $H_{\alpha}$ is formally defined as $\varphi_{\mp}=W_{ \pm}\left(H_{\alpha}, H_{0}\right) \varphi_{0}$ by making use of the intertwining property of wave operators. However this definition does not have the rigorous meaning, because $\varphi_{0}$ is not in $L^{2}\left(\mathbf{R}^{2}\right)$. To give the precise definition, we make use of the expansion formula $(3.1)$ for $\varphi_{0}(x ; \lambda, \omega)$ and of the well known asymptotic formula of Bessel functions

$$
J_{p}(r)=(2 / \pi)^{1 / 2} r^{-1 / 2} \cos (r-(2 p+1) \pi / 4)\left(1+g_{m}(r)\right)+O\left(r^{-m}\right)
$$


as $r \rightarrow \infty$ for any $m \gg 1$ large enough, where $g_{m}(r)$ obeys $(d / d r)^{k} g_{m}(r)=$ $O\left(r^{-1-k}\right)$. If we set

$$
e_{\mp l}(r)=\exp ( \pm i|l| \pi / 2) J_{|l|}(r)-\exp ( \pm i \nu \pi / 2) J_{\nu}(r)
$$

with $\nu=|l-\alpha|$ again, then it follows from (3.3) that

$$
e_{\mp l}(r)=\exp (\mp i r)\left(C_{\mp l} r^{-1 / 2}+O\left(r^{-3 / 2}\right)\right)+\exp ( \pm i r) O\left(r^{-3 / 2}\right)
$$

for some constant $C_{\mp l}$. Hence $e_{-l}(r)$ satisfies the incoming radiation condition $e_{-l}^{\prime}+i e_{-l}=O\left(r^{-3 / 2}\right)$ at infinity, while $e_{+l}(r)$ satisfies the outgoing radiation condition $e_{+l}^{\prime}-i e_{+l}=O\left(r^{-3 / 2}\right)$. Thus, if we make use of the simple relation

$$
\exp (i l \theta(x ;-\omega))=\exp (i|l| \pi+i l \theta(x ; \omega))
$$

between azimuth angles $\theta(x ; \omega)$ and $\theta(x ;-\omega)$ and if we take account of the expansion formula (3.1), then the eigenfunction $\varphi_{\mp}$ is defined by

$$
\varphi_{\mp}(x ; \lambda, \omega)=\sum_{l \in \mathbf{Z}} \exp ( \pm i \nu \pi / 2) \exp (i l \theta(x ; \pm \omega)) J_{\nu}(\sqrt{\lambda}|x|) .
$$

As is easily seen, this series converges locally uniformly and $\varphi_{\mp}$ satisfies the equation $H_{\alpha} \varphi_{\mp}=\lambda \varphi_{\mp}$.

As stated previously, the ordinary wave operators $W_{ \pm}\left(H, H_{0}\right)$ exist and are asymptotically complete, although $H-H_{0}$ is a perturbation of longrange class. Hence the scattering operator

$$
S\left(H, H_{0}\right)=W_{+}^{*}\left(H, H_{0}\right) W_{-}\left(H, H_{0}\right): L^{2}\left(\mathbf{R}^{2}\right) \longrightarrow L^{2}\left(\mathbf{R}^{2}\right)
$$

can be defined as a unitary operator and it has the direct integral decomposition

$$
S\left(H, H_{0}\right) \simeq \mathcal{F}_{0} S\left(H, H_{0}\right) \mathcal{F}_{0}^{*}=\int_{0}^{\infty} \oplus S\left(\lambda ; H, H_{0}\right) d \lambda,
$$

where the fibre $S\left(\lambda ; H, H_{0}\right): L^{2}\left(S^{1}\right) \rightarrow L^{2}\left(S^{1}\right)$ is called the scattering matrix at energy $\lambda>0$ and it acts as

$$
\left(\mathcal{F}_{0} S\left(H, H_{0}\right) u\right)(\lambda, \omega)=\left(S\left(\lambda ; H, H_{0}\right)\left(\mathcal{F}_{0} u\right)(\lambda, \cdot)\right)(\omega)
$$


for $u \in L^{2}\left(\mathbf{R}^{2}\right)$. By the chain rule of wave operators, we have

$$
W_{ \pm}\left(H, H_{0}\right)=W_{ \pm}\left(H, H_{\alpha}\right) W_{ \pm}\left(H_{\alpha}, H_{0}\right)
$$

and hence

$$
S\left(H, H_{0}\right)=W_{+}^{*}\left(H_{\alpha}, H_{0}\right) S\left(H, H_{\alpha}\right) W_{-}\left(H_{\alpha}, H_{0}\right),
$$

where $S\left(H, H_{\alpha}\right)=W_{+}^{*}\left(H, H_{\alpha}\right) W_{-}\left(H, H_{\alpha}\right)$. The existence and completeness of wave operators $W_{ \pm}\left(H, H_{\alpha}\right)$ follow from those of $W_{ \pm}\left(H_{\alpha}, H_{0}\right)$ and $W_{ \pm}\left(H, H_{0}\right)$ at once.

We shall derive the integral kernel of scattering matrix $S\left(\lambda ; H, H_{0}\right)$. Before doing this, we make a brief review on the spectral properties of $H$, which are required in the argument below. The operator $H$ is known to have the following spectral properties $([6])$ : (1) $H$ has no positive bound state energies; (2) The resolvents $R(\lambda \pm i \varepsilon ; H)=(H-\lambda \mp i \varepsilon)^{-1}, \varepsilon>0$, have the boundary values to the positive axis

$$
R(\lambda \pm i 0 ; H)=\lim _{\varepsilon \rightarrow 0} R(\lambda \pm i \varepsilon ; H), \quad \lambda>0
$$

as an operator from the weighted $L^{2}$ space $L_{s}^{2}\left(\mathbf{R}^{2}\right)=L^{2}\left(\mathbf{R}^{2} ;\langle x\rangle^{2 s} d x\right)$ into $L_{-s}^{2}\left(\mathbf{R}^{2}\right)$ for $s>1 / 2$, where $\langle x\rangle=\left(1+|x|^{2}\right)^{1 / 2}$.

We now denote by $($,$) or (,)_{L^{2}\left(\mathbf{R}^{2}\right)}$ the $L^{2}$ scalar product in $L^{2}\left(\mathbf{R}^{2}\right)$. To represent the scattering kernel, we consider the quantity

$$
S\left(\omega^{\prime}, \omega ; \lambda^{\prime}, \lambda\right)=2^{-1}(2 \pi)^{-2}\left(S\left(H, H_{0}\right) \varphi_{0}(\cdot ; \lambda, \omega), \varphi_{0}\left(\cdot ; \lambda^{\prime}, \omega^{\prime}\right)\right),
$$

which is a formal representation for the integral kernel of the operator

$$
\mathcal{F}_{0} S\left(H, H_{0}\right) \mathcal{F}_{0}^{*}: L^{2}((0, \infty) ; d \lambda) \otimes L^{2}\left(S^{1}\right) \longrightarrow L^{2}((0, \infty) ; d \lambda) \otimes L^{2}\left(S^{1}\right)
$$

If we make use of the formal relation $\varphi_{\mp}=W_{ \pm}\left(H_{\alpha}, H_{0}\right) \varphi_{0}$, then it follows from (3.5) that $S\left(\omega^{\prime}, \omega ; \lambda^{\prime}, \lambda\right)$ is decomposed into the sum of two terms

$$
S\left(\omega^{\prime}, \omega ; \lambda^{\prime}, \lambda\right)=S_{0}\left(\omega^{\prime}, \omega ; \lambda^{\prime}, \lambda\right)+S_{1}\left(\omega^{\prime}, \omega ; \lambda^{\prime}, \lambda\right),
$$

where $S_{0}=2^{-1}(2 \pi)^{-2}\left(\varphi_{+}(\cdot ; \lambda, \omega), \varphi_{-}\left(\cdot ; \lambda^{\prime}, \omega^{\prime}\right)\right)$ and

$$
S_{1}=2^{-1}(2 \pi)^{-2}\left(\left(S\left(H, H_{\alpha}\right)-\mathrm{Id}\right) \varphi_{+}(\cdot ; \lambda, \omega), \varphi_{-}\left(\cdot ; \lambda^{\prime}, \omega^{\prime}\right)\right) .
$$


We often identify the coordinates over $S^{1}$ with the azimuth angles from the positive $x_{1}$ axis in the argument below. We first calculate $S_{0}\left(\omega^{\prime}, \omega ; \lambda^{\prime}, \lambda\right)$. Recall the representation (3.4) for $\varphi_{\mp}(x ; \lambda, \omega)$. If we take account of the simple relation

$$
\exp \left(i l\left(\theta(x ;-\omega)-\theta\left(x ; \omega^{\prime}\right)\right)\right)=\exp \left(i l\left(\omega^{\prime}-\omega+\pi\right)\right)
$$

then we have

$$
\begin{aligned}
S_{0}=2^{-1}(2 \pi)^{-1} & \sum_{l \in \mathbf{Z}} \exp (i(l-\nu) \pi) \exp \left(i l\left(\omega^{\prime}-\omega\right)\right) \\
& \times \int_{0}^{\infty} r J_{\nu}(\sqrt{\lambda} r) J_{\nu}\left(\sqrt{\lambda^{\prime}} r\right) d r
\end{aligned}
$$

The integral on the right side equals

$$
\int_{0}^{\infty} r J_{\nu}(\sqrt{\lambda} r) J_{\nu}\left(\sqrt{\lambda^{\prime}} r\right) d r=\lambda^{-1 / 2} \delta\left(\sqrt{\lambda^{\prime}}-\sqrt{\lambda}\right)=2 \delta\left(\lambda^{\prime}-\lambda\right)
$$

(see $[5$, p.73]), and hence

$$
S_{0}=(2 \pi)^{-1}\left(\sum_{l \in \mathbf{Z}} \exp (i(l-\nu) \pi) \exp \left(i l\left(\omega^{\prime}-\omega\right)\right)\right) \delta\left(\lambda^{\prime}-\lambda\right) .
$$

We can further show $([11])$ that

$$
\sum_{l \in \mathbf{Z}} \exp (i(l-\nu) \pi) \exp (i l \theta)=2 \pi\left(\cos \alpha \pi \delta(\theta)-(i \sin \alpha \pi / \pi) F_{0}(\theta)\right)
$$

for $\alpha, 0<\alpha<1$, where $F_{0}(\theta)$ is again defined by $F(\theta)=$ v.p. $e^{i \theta} /\left(e^{i \theta}-1\right)$. Thus we obtain

$$
S_{0}=\left(\cos \alpha \pi \delta\left(\omega^{\prime}-\omega\right)-(i \sin \alpha \pi / \pi) F_{0}\left(\omega^{\prime}-\omega\right)\right) \delta\left(\lambda^{\prime}-\lambda\right) .
$$

Next we calculate the second term $S_{1}\left(\omega^{\prime}, \omega ; \lambda^{\prime}, \lambda\right)$. Let $\chi(s) \in C_{0}^{\infty}([0, \infty))$ be the basic cut-off function with property $(2.2)$. We set $\chi_{\infty M}(x)=\chi_{\infty M}(r)=$ $1-\chi(r / M)$ for $M>2, M$ being fixed. The function $\chi_{\infty M}$ has support in $|x|>M$ and take the value $\chi_{\infty M}=1$ for $|x|>2 M$, so that $H=H_{\alpha}$ on the support of $\chi_{\infty M}$ by $(2.1)$ and (2.2). Since

$$
\left\|\left(1-\chi_{\infty M}\right) \exp (-i t H) W_{-}\left(H, H_{\alpha}\right) u\right\|_{L^{2}\left(\mathbf{R}^{2}\right)} \rightarrow 0, \quad t \rightarrow \pm \infty,
$$


for $u \in L^{2}\left(\mathbf{R}^{2}\right)$, we have

$$
\begin{aligned}
S\left(H, H_{\alpha}\right)-\mathrm{Id} & =\left.\exp \left(i t H_{\alpha}\right) \chi_{\infty M} \exp (-i t H) W_{-}\left(H, H_{\alpha}\right)\right|_{t=-\infty} ^{t=\infty} \\
& =i \int \exp \left(i t H_{\alpha}\right) D_{M} W_{-}\left(H, H_{\alpha}\right) \exp \left(-i t H_{\alpha}\right) d t
\end{aligned}
$$

by the intertwining property, where

$$
D_{M}=H_{\alpha} \chi_{\infty M}-\chi_{\infty M} H=H_{\alpha} \chi_{\infty M}-\chi_{\infty M} H_{\alpha}=\left[H_{\alpha}, \chi_{\infty M}\right]
$$

If we further make use of relation $\exp \left(-i t H_{\alpha}\right) \varphi_{\mp}=\exp (-i t \lambda) \varphi_{\mp}$ and of formula

$$
\int \exp \left(i t\left(\lambda^{\prime}-\lambda\right)\right) d t=2 \pi \delta\left(\lambda^{\prime}-\lambda\right)
$$

then we obtain

$$
S_{1}\left(\omega^{\prime}, \omega ; \lambda^{\prime}, \lambda\right)=(i / 4 \pi) I\left(\omega^{\prime}, \omega ; \lambda^{\prime}, \lambda\right) \delta\left(\lambda^{\prime}-\lambda\right)
$$

by a formal computation, where

$$
I\left(\omega^{\prime}, \omega ; \lambda^{\prime}, \lambda\right)=-\left(W_{-}\left(H, H_{\alpha}\right) \varphi_{+}(\cdot ; \lambda, \omega), D_{M} \varphi_{-}\left(\cdot ; \lambda^{\prime}, \omega^{\prime}\right)\right)
$$

because of relation $D_{M}^{*}=-D_{M}$. The wave operator $W_{-}\left(H, H_{\alpha}\right)$ is written in the integral form

$$
W_{-}\left(H, H_{\alpha}\right)=\chi_{\infty M}-i \int_{-\infty}^{0} \exp (i t H) D_{M} \exp \left(-i t H_{\alpha}\right) d t
$$

Hence it follows again by a formal computation that

$$
S_{1}=(i / 4 \pi) I\left(\omega^{\prime}, \omega ; \lambda\right) \delta\left(\lambda^{\prime}-\lambda\right),
$$

where $I\left(\omega^{\prime}, \omega ; \lambda\right)$ is given by

$$
I\left(\omega^{\prime}, \omega ; \lambda\right)=\left(\left(-\chi_{\infty M}+R(\lambda+i 0 ; H) D_{M}\right) \varphi_{+}(\cdot ; \lambda, \omega), D_{M} \varphi_{-}\left(\cdot ; \lambda, \omega^{\prime}\right)\right) .
$$

We combine the two representations above to obtain that the integral kernel $S\left(\omega^{\prime}, \omega ; \lambda\right)$ of scatterimg matrix $S\left(\lambda ; H, H_{0}\right): L^{2}\left(S^{1}\right) \rightarrow L^{2}\left(S^{1}\right)$ is represented as

$$
S\left(\omega^{\prime}, \omega ; \lambda\right)=S_{0}\left(\omega^{\prime}, \omega ; \lambda\right)+S_{1}\left(\omega^{\prime}, \omega ; \lambda\right)
$$


where $S_{0}=\cos \alpha \pi \delta\left(\omega^{\prime}-\omega\right)-(i \sin \alpha \pi / \pi) F_{0}\left(\omega^{\prime}-\omega\right)$ and

$$
S_{1}=(i / 4 \pi)\left(\left(-\chi_{\infty M}+R(\lambda+i 0 ; H) D_{M}\right) \varphi_{+}(\cdot ; \lambda, \omega), D_{M} \varphi_{-}\left(\cdot ; \lambda, \omega^{\prime}\right)\right) .
$$

The scattering amplitude $f\left(\omega \rightarrow \omega^{\prime} ; \lambda\right)$ in question for the pair $\left(H, H_{0}\right)$ is now defined by

$$
f\left(\omega \rightarrow \omega^{\prime} ; \lambda\right)=c(\lambda)\left(S\left(\omega^{\prime}, \omega ; \lambda\right)-\delta\left(\omega^{\prime}-\omega\right)\right)
$$

with $c(\lambda)=(2 \pi / i \sqrt{\lambda})^{1 / 2}$ again. By $(3.4),\left(D_{M} \varphi_{-}\right)(x ; \lambda, \omega)=o(1)$ as $\lambda \rightarrow 0$ and hence

$$
\left(\chi_{\infty M} \varphi_{+}(\cdot ; \lambda, \omega), D_{M} \varphi_{-}\left(\cdot ; \lambda, \omega^{\prime}\right)\right) \rightarrow 0, \quad \lambda \rightarrow 0 .
$$

Thus we obtain the following proposition.

Proposition 3.1. Let the notations be as above and let, in particular, $c(\lambda)$ denote $c(\lambda)=(2 \pi / i \sqrt{\lambda})^{1 / 2}$. Then the scattering amplitude $f\left(\omega \rightarrow \omega^{\prime} ; \lambda\right)$ behaves like

$$
f\left(\omega \rightarrow \omega^{\prime} ; \lambda\right)=c(\lambda)\left(f_{\alpha}\left(\omega^{\prime}-\omega\right)+g_{\alpha}\left(\omega \rightarrow \omega^{\prime} ; \lambda\right)+o(1)\right), \quad \lambda \rightarrow 0,
$$

where

$$
f_{\alpha}\left(\omega^{\prime}-\omega\right)=(\cos \alpha \pi-1) \delta\left(\omega^{\prime}-\omega\right)-(i \sin \alpha \pi / \pi) F_{0}\left(\omega^{\prime}-\omega\right)
$$

with $F_{0}(\theta)=$ v.p. $e^{i \theta} /\left(e^{i \theta}-1\right)$, and

$$
g_{\alpha}\left(\omega \rightarrow \omega^{\prime} ; \lambda\right)=(i / 4 \pi)\left(R(\lambda+i 0 ; H) D_{M} \varphi_{+}(\cdot ; \lambda, \omega), D_{M} \varphi_{-}\left(\cdot ; \lambda, \omega^{\prime}\right)\right)
$$

For later reference, we here discuss the relation between the scattering at low energy and the scattering by magnetic fields with small support. Let $b(x), V(x) \in C_{0}^{\infty}\left(\mathbf{R}^{2}\right)$ be the same electric and magnetic fields as above and let $A(x)$ be also the same magnetic potential associated with field $b(x)$. We consider

$$
b_{\varepsilon}(x)=\varepsilon^{-2} b(x / \varepsilon), \quad V_{\varepsilon}(x)=\varepsilon^{-2} V(x / \varepsilon), \quad 0<\varepsilon \ll 1,
$$

as magnetic and electric fields with small support, respectively. If we further set $A_{\varepsilon}(x)=\varepsilon^{-1} A(x / \varepsilon)$, then $\nabla \times A_{\varepsilon}=b_{\varepsilon}$ and hence $A_{\varepsilon}$ becomes the magnetic potential associated with $b_{\varepsilon}(x)$. We now define the Hamiltonian $H_{\varepsilon}$ by

$$
H_{\varepsilon}=H\left(A_{\varepsilon}, V_{\varepsilon}\right)=\left(-i \nabla-A_{\varepsilon}\right)^{2}+V_{\varepsilon}
$$


and denote by $f_{\varepsilon}\left(\omega \rightarrow \omega^{\prime} ; \lambda\right)$ the scattering amplitude for the pair $\left(H_{\varepsilon}, H_{0}\right)$. The eigenfunction of $H_{\alpha}$ satisfies the invariance relation $\varphi_{\mp}\left(x / \varepsilon ; \lambda \varepsilon^{2}, \omega\right)=$ $\varphi_{\mp}(x ; \lambda, \omega)$. If $u=u(x)$ obeys the equation $H_{\varepsilon} u-\lambda u=f$, then $u_{\varepsilon}(x)=$ $u(\varepsilon x)$ solves the equation $H u_{\varepsilon}-\lambda \varepsilon^{2} u_{\varepsilon}=\varepsilon^{2} f_{\varepsilon}$ with $f_{\varepsilon}(x)=f(\varepsilon x)$, so that

$$
\left(R\left(\lambda+i 0 ; H_{\varepsilon}\right) f\right)(x)=\varepsilon^{2}\left(R\left(\lambda \varepsilon^{2}+i 0 ; H\right) f_{\varepsilon}\right)(x / \varepsilon)
$$

for $f \in C_{0}^{\infty}\left(\mathbf{R}^{2}\right)$. If we take these facts into account and if we make a change $x / \varepsilon \rightarrow x$ of variables, we have the relation

$$
f_{\varepsilon}\left(\omega \rightarrow \omega^{\prime} ; \lambda\right)=\sqrt{\varepsilon} f\left(\omega \rightarrow \omega^{\prime} ; \lambda \varepsilon^{2}\right)
$$

and hence Proposition 3.1 yields the behavior as $\varepsilon \rightarrow 0$ of $f_{\varepsilon}\left(\omega \rightarrow \omega^{\prime} ; \lambda\right)$.

Corollary 3.1. Assume that $\lambda>0$ is fixed. Let $f_{\alpha}\left(\omega^{\prime}-\omega\right)$ and $g_{\alpha}\left(\omega \rightarrow \omega^{\prime} ; \lambda\right)$ be as in Proposition 3.1. Then $f_{\varepsilon}\left(\omega \rightarrow \omega^{\prime} ; \lambda\right)$ behaves like

$$
f_{\varepsilon}\left(\omega \rightarrow \omega^{\prime} ; \lambda\right)=c(\lambda)\left(f_{\alpha}\left(\omega^{\prime}-\omega\right)+g_{\alpha}\left(\omega \rightarrow \omega^{\prime} ; \lambda \varepsilon^{2}\right)\right)+o(1), \quad \varepsilon \rightarrow 0 .
$$

\section{$\S 4$. Preliminaries for resolvent estimate I}

The problem is now reduced to analysing the behavior at low energy of the resolvent $\mathbf{R}(\lambda+i 0 ; H)$. To do this, we introduce the auxiliary operator

$$
K_{\alpha}=\left(-i \nabla-\chi_{\infty} B_{\alpha}\right)^{2}
$$

as stated in section 1 , where $\chi_{\infty}=\chi_{\infty}(r)=1-\chi(r)$. The function $\chi_{\infty}$ has support in $r>1$ and takes the value $\chi_{\infty}=1$ for $r>2$. The operator $K_{\alpha}$ has the same domain as the original operator $H$, and it follows from (2.3) that the difference $W=H-K_{\alpha}$ is a first order differential operator having smooth coefficients with support in $\left\{x \in \mathbf{R}^{2}:|x|<2\right\}$. In addition, $K_{\alpha}$ admits the partial wave expansion

$$
K_{\alpha} \simeq U K_{\alpha} U^{*}=\sum_{l \in \mathbf{Z}} \oplus\left(K_{l \alpha} \otimes \mathrm{Id}\right)
$$

by the unitary mapping $U$ defined by $(3.2)$, where

$$
K_{l \alpha}=\Pi^{*} \Pi+\left(l-\alpha \chi_{\infty}\right)^{2} r^{-2}, \quad \Pi=d / d r-1 / 2 r,
$$

is self-adjoint in $L^{2}\left(\mathbf{R}_{+}\right)=L^{2}((0, \infty) ; d r)$ with domain

$$
\mathcal{D}\left(K_{l \alpha}\right)=\left\{u \in L^{2}\left(\mathbf{R}_{+}\right): K_{l \alpha} u \in L^{2}\left(\mathbf{R}_{+}\right), \lim _{r \rightarrow 0} r^{-1 / 2} u(r)<\infty\right\} .
$$


If $l \neq 0$, then the above limit vanishes $\lim _{r \rightarrow 0} r^{-1 / 2} u(r)=0$.

As preliminary steps, the present and next sections are devoted to the study on the behavior at low energy of $R\left(\lambda+i 0 ; K_{\alpha}\right)$. We now fix an integer $l_{0} \gg 1$ large enough, and we further introduce another auxiliary operators $X_{l}, l \in \mathbf{Z}$, acting on $L^{2}\left(\mathbf{R}_{+}\right)$. We define $X_{l}$ as

$$
X_{l}=\Pi^{*} \Pi+q_{l}
$$

for $l$ with $|l| \leq l_{0}$, where

$$
q_{l}(r)=l^{2} r^{-2} \quad \text { for } 0<r<1, \quad q_{l}(r)=\nu^{2} r^{-2} \quad \text { for } r>1
$$

and $\nu$ again denotes $\nu=|l-\alpha|$. On the other hand, if $|l|>l_{0}$, then we define

$$
X_{l}=\Pi^{*} \Pi+\nu^{2} r^{-2} .
$$

Each operator $X_{l}$ is self-adjoint in $L^{2}\left(\mathbf{R}_{+}\right)$with the same domain as $K_{l \alpha}$. This holds true even for $|l|>l_{0} \gg 1$. In fact, we have

$$
\mathcal{D}\left(K_{l \alpha}\right)=\mathcal{D}\left(X_{l}\right)=\left\{u \in L^{2}\left(\mathbf{R}_{+}\right): u \in H^{2}\left(\mathbf{R}_{+}\right), r^{-2} u \in L^{2}\left(\mathbf{R}_{+}\right)\right\}
$$

for $|l|>l_{0} \gg 1$. Hence $R\left(k^{2}+i 0 ; K_{l \alpha}\right), \lambda=k^{2}$, is represented as

$$
R\left(k^{2}+i 0 ; K_{l \alpha}\right)=R\left(k^{2}+i 0 ; X_{l}\right)\left(\mathrm{Id}+\left(K_{l \alpha}-X_{l}\right) R\left(k^{2}+i 0 ; X_{l}\right)\right)^{-1}
$$

by use of the resolvent identity.

As the first step, we here study the behavior as $k \rightarrow 0$ of $R\left(k^{2}+i 0 ; X_{l}\right)$. Throughout the section, we work in $L^{2}\left(\mathbf{R}_{+}\right)$, and we denote by $($,$) or$ $(,)_{L^{2}\left(\mathbf{R}_{+}\right)}$the $L^{2}$ scalar product and by \|\| the $L^{2}$ norm. The same notation || || is often used to denote the operator norm of bounded operators acting on $L^{2}\left(\mathbf{R}_{+}\right)$. We further introduce several new notations required in the argument below. Let $L \gg 1$ be fixed large enough. We write $I_{L}$ for the interval $[0,2 L)$, and denote by $B\left(L_{\text {com }}^{2}\left(I_{L}\right) \rightarrow L_{\text {loc }}^{2}\left(\mathbf{R}_{+}\right)\right)$the class of all operators $T$ such that $\chi_{R} T: L_{\text {com }}^{2}\left(I_{L}\right) \rightarrow L^{2}\left(\mathbf{R}_{+}\right), \chi_{R}=\chi(r / R)$, is bounded for any $R>0$, when it is restricted to the subspace

$$
L_{\mathrm{com}}^{2}\left(I_{L}\right)=\left\{f \in L^{2}\left(\mathbf{R}_{+}\right): \operatorname{supp} f \subset[0,2 L)\right\} .
$$

We say that $T(k) \in B\left(L_{\text {com }}^{2}\left(I_{L}\right) \rightarrow L_{\text {loc }}^{2}\left(\mathbf{R}_{+}\right)\right)$is of class $O p\left(O\left(k^{\gamma}\right)\right)$, if it obeys the bound $\left\|\chi_{R} T(k)\right\|=O\left(k^{\gamma}\right)$ as $k \rightarrow 0$. We sometimes use the same 
notation $T(k) \in O p\left(O\left(k^{\gamma}\right)\right)$ for bounded operator $T(k) \in B(\mathcal{X} \rightarrow \mathcal{Y})$ from Hilbert space $\mathcal{X}$ to $\mathcal{Y}$, when $T(k)$ obeys the bound $\|T(k)\|=O\left(k^{\gamma}\right)$.

4.1. The argument is divided into the two cases $|l| \leq l_{0}$ and $|l|>l_{0}$. We first deal with the case $|l| \leq l_{0}$. We consider the homogeneous equation $X_{l} u=0$. This equation has the following pair $\left(u_{l}, v_{l}\right)$ with the Wronskian normalized by $W\left(u_{l}, v_{l}\right)=-1$ as linearly independent solutions: If $l=0$, then

$$
\begin{aligned}
& u_{0}(r)= \begin{cases}\nu^{-1 / 2} r^{1 / 2} & r \leq 1, \\
\frac{1}{2} \nu^{-1 / 2}\left(r^{\nu+1 / 2}+r^{-\nu+1 / 2}\right) & r>1,\end{cases} \\
& v_{0}(r)= \begin{cases}\nu^{-1 / 2}\left(r^{1 / 2}-\nu r^{1 / 2} \log r\right) & r \leq 1, \\
\nu^{-1 / 2} r^{-\nu+1 / 2} & r>1,\end{cases}
\end{aligned}
$$

with $\nu=|l-\alpha|=\alpha$ for $l=0$, and if $|l| \leq l_{0}, l \neq 0$, then

$$
\begin{aligned}
& u_{l}(r)= \begin{cases}(|l|+\nu)^{-1 / 2} r^{|l|+1 / 2} & r \leq 1, \\
\frac{1}{2}(|l|+\nu)^{-1 / 2}\left((1+|l| / \nu) r^{\nu+1 / 2}+(1-|l| / \nu) r^{-\nu+1 / 2}\right) & r>1,\end{cases} \\
& v_{l}(r)= \begin{cases}\frac{1}{2}(|l|+\nu)^{-1 / 2}\left((1-\nu /|l|) r^{|l|+1 / 2}+(1+\nu /|l|) r^{-|l|+1 / 2}\right) & r \leq 1, \\
(|l|+\nu)^{-1 / 2} r^{-\nu+1 / 2} & r>1 .\end{cases}
\end{aligned}
$$

Let $E_{l 0}$ be defined by

$$
\left(E_{l 0} f\right)(r)=\int_{0}^{\infty} E_{l 0}(r, p) f(p) d p
$$

where

$$
E_{l 0}(r, p)=u_{l}(r \wedge p) v_{l}(r \vee p), \quad r \wedge p=\min (r, p), r \vee p=\max (r, p)
$$

Then $u(r)=E_{l 0} f(r)$ yields a unique solution to

$$
X_{l} u=f, \quad \lim _{r \rightarrow 0} r^{-1 / 2} u(r)<\infty,
$$

for given $f \in L_{\text {com }}^{2}\left(I_{L}\right)$. If, in particular, $l \neq 0,1$, then it follows that $E_{l 0} f \in L^{2}\left(\mathbf{R}_{+}\right)$. The kernel $E_{l 0}(r, p)$ is symmetric with respect to $(r, p)$ and hence $E_{l 0}$ is formally self-adjoint $E_{l 0}^{*}=E_{l 0}$ in the sense that

$$
\left(E_{l 0} f, g\right)=\left(f, E_{l 0} g\right), \quad f, g \in L_{\mathrm{com}}^{2}\left(I_{L}\right)
$$

The proposition below plays an important role in the analysis at low energy of the resolvent $R(\lambda+i 0 ; H)$. 
Proposition 4.1. Assume that $|l| \leq l_{0}$. Then one has:

(1) The resolvent $R\left(k^{2} \pm i 0 ; X_{l}\right), k>0$, has the following asymptotic expansion in $B\left(L_{\mathrm{com}}^{2}\left(I_{L}\right) \rightarrow L_{\mathrm{loc}}^{2}\left(\mathbf{R}_{+}\right)\right)$: If $l=0$ or 1 , then

$$
R\left(k^{2} \pm i 0 ; X_{l}\right)=E_{l 0}+E_{l 1}( \pm k)+k^{2} E_{l 2}+O p\left(o\left(k^{2}\right)\right), \quad k \rightarrow 0
$$

and if $l \neq 0,1$, then

$$
R\left(k^{2} \pm i 0 ; X_{l}\right)=E_{l 0}+k^{2} E_{l 2}+O p\left(o\left(k^{2}\right)\right), \quad k \rightarrow 0 .
$$

(2) $E_{l 0}$ and $E_{l 2}$ are formally self-adjoint in the above sense.

(3) Let $\left(u_{l}, v_{l}\right)$ be as above. Then $E_{l 1}(k), l=0,1$, belongs to $O p\left(O\left(k^{2 \nu}\right)\right)$ and satisfies $E_{l 1}^{*}(k)=E_{l 1}(-k)$. This operator acts as

$$
E_{l 1}(k) f(r)=\gamma_{l}(k)\left(f, u_{l}\right) u_{l}(r)
$$

for $f \in L_{\mathrm{com}}^{2}\left(I_{L}\right)$, where

$$
\gamma_{l}(k)=2 \nu(l-\nu)^{-1} \beta_{l}(k)\left(1+\beta_{l}(k)\right)^{-1}
$$

with

$$
\beta_{l}(k)=-((l-\nu) \Gamma(1-\nu) /(l+\nu) \Gamma(1+\nu)) e^{-i \nu \pi}(k / 2)^{2 \nu} .
$$

Remarks. (1) The argument below does not require the explicit representation for the integral kernel of operator $E_{l 2}$. (2) The proposition remains true for $R\left(\zeta ; X_{l}\right)$ with $\operatorname{Im} \sqrt{\zeta} \geq 0$. (3) Since $\bar{\gamma}_{l}(k)=\gamma_{l}(-k)$, $E_{l 1}^{*}(k)=E_{l 1}(-k)$ and hence the formal self-adjointness of $E_{l 2}$ follows at once.

We proceed with the argument, accepting this proposition as proved. We will prove it in the last section (section 9). The proof is direct but rather lengthy, using the asymptotic formulas of Bessel functions.

4.2. Next we consider the case $|l|>l_{0}$. The homogeneous equation $X_{l} u=0$ has the following pair $\left(u_{l}, v_{l}\right)$ normalized by $W\left(u_{l}, v_{l}\right)=-1$ as linearly independent solutions:

$$
u_{l}(r)=(2 \nu)^{-1 / 2} r^{\nu+1 / 2}, \quad v_{l}(r)=(2 \nu)^{-1 / 2} r^{-\nu+1 / 2} .
$$

The integral operator $E_{l 0}$ defined as in (4.3) yields a unique solution to

$$
X_{l} u=f, \quad \lim _{r \rightarrow 0} r^{-1 / 2} u(r)=0,
$$

for given $f \in L_{\text {com }}^{2}\left(I_{L}\right)$, and the solution $E_{l 0} f$ belongs to $L^{2}\left(\mathbf{R}_{+}\right)$. 
Lemma 4.1. $\quad r^{-2} E_{l 0}: L_{\text {com }}^{2}\left(I_{L}\right) \rightarrow L_{\text {loc }}^{2}\left(\mathbf{R}_{+}\right)$is defined and it satisfies

$$
\left\|\chi_{R} r^{-2} E_{l 0}\right\|=O\left(|l|^{-2}\right), \quad|l| \rightarrow \infty .
$$

Proof. As stated above, $u=E_{l 0} f$ is a $L^{2}$ solution to $X_{l} u=f$ for $f \in$ $L_{\text {com }}^{2}\left(I_{L}\right)$. By the density argument, we may assume that $f \in C_{0}^{\infty}((0,2 L))$. Then $u \in C^{\infty}\left(\mathbf{R}_{+}\right)$behaves like $u(r)=O\left(r^{\nu+1 / 2}\right)$ as $r \rightarrow 0$, and hence $r^{-2} u \in L^{2}\left(\mathbf{R}_{+}\right)$. We take the scalar product between the equation $X_{l} u=f$ and $r^{-2} u$. If $|l| \gg 1$, a simple calculation using integration by parts shows that $|l|^{2}\left\|r^{-2} u\right\| \leq c\|f\|$ for $c>0$ independent of $l$. This proves the lemma.

If we take account of the relation $W\left(J_{\nu}, H_{\nu}\right)(z)=2 i / \pi z$, then the integral kernel $R_{l}(r, p ; k)$ of $R\left(k^{2}+i 0 ; X_{l}\right), k>0$, is given by

$$
R_{l}(r, p ; k)=(i \pi / 2) r^{1 / 2} p^{1 / 2} J_{\nu}(k(r \wedge p)) H_{\nu}(k(r \vee p)),
$$

where $H_{\nu}(z)=H_{\nu}^{(1)}(z)$ is the first kind of the Hankel function of order $\nu$. The Hankel function $H_{\nu}(z)$ is represented by the formula

$$
H_{\nu}(z)=J_{\nu}(z)+i N_{\nu}(z)=(i / \sin \nu \pi)\left(e^{-i \nu \pi} J_{\nu}(z)-J_{-\nu}(z)\right)
$$

in terms of the Bessel functions $J_{ \pm \nu}(z)$, while the Bessel functions $J_{ \pm \nu}(z)$ are expanded as

$$
J_{ \pm \nu}(z)=\left(\frac{z}{2}\right)^{ \pm \nu} \sum_{n=0}^{\infty} \frac{(-1)^{n}(z / 2)^{2 n}}{\Gamma(n+1) \Gamma(n+1 \pm \nu)} .
$$

Since $\Gamma(1+\nu) \Gamma(1-\nu)=\nu \pi / \sin \nu \pi, R_{l}(r, p ; k)$ is expanded in the form

$$
R_{l}(r, p ; k)=E_{l 0}(r, p)+k^{2} E_{l 2}(r, p)+E_{l 3}(r, p ; k)
$$

by use of the above formulas, where $E_{l 2}(r, p)$ and $E_{l 3}(r, p ; k)$ obey

$$
\left|E_{l 2}(r, p)\right|=O\left(|l|^{-1}\right) r^{1 / 2} p^{1 / 2}(r \wedge p)^{\nu}(r \vee p)^{2-\nu}
$$

as $|l| \rightarrow \infty$, and

$$
\left|E_{l 3}(r, p ; k)\right|=|l|^{-1} r^{1 / 2} p^{1 / 2}(r \wedge p)^{\nu}\left(O\left(k^{2 \nu}\right)(r \vee p)^{\nu}+O\left(k^{4}\right)(r \vee p)^{4-\nu}\right)
$$

as $k \rightarrow 0$, the order estimates being locally uniform in $r$ and $p$. 
LEMMA 4.2. Let $E_{l 2}$ and $E_{l 3}(k)$ be the integral operators with kernels $E_{l 2}(r, p)$ and $E_{l 3}(r, p ; k)$, respectively. Then one has:

(1) $r^{-2} E_{l 2}: L_{\text {com }}^{2}\left(I_{L}\right) \rightarrow L_{\text {loc }}^{2}\left(\mathbf{R}_{+}\right)$obeys

$$
\left\|\chi_{R} r^{-2} E_{l 2}\right\|=O\left(|l|^{-1}\right), \quad|l| \rightarrow \infty .
$$

(2) $r^{-2} E_{l 3}(k): L_{\text {com }}^{2}\left(I_{L}\right) \rightarrow L_{\mathrm{loc}}^{2}\left(\mathbf{R}_{+}\right)$is of class $O p\left(o\left(k^{2}\right)\right)$ and

$$
\left\|\chi_{R} r^{-2} E_{l 3}(k)\right\|=|l|^{-1} o\left(k^{2}\right), \quad k \rightarrow 0,
$$

uniformly in $|l| \gg 1$.

Proof. If we use the above estimate for $E_{l 2}(r, p)$, then the Schwarz inequality implies that

$$
\left|r^{-2}\left(E_{l 2} f\right)(r)\right| \leq c|l|^{-1} r^{3 / 2}\|f\|
$$

for $f \in L_{\text {com }}^{2}\left(I_{L}\right)$. This proves (1). The second statement is verified in a similar way.

We combine Lemmas 4.1 and 4.2 to obtain the following proposition.

Proposition 4.2. Assume that $|l|>l_{0} \gg 1$. Then one has:

(1) $R\left(k^{2} \pm i 0 ; X_{l}\right), k>0$, has the asymptotic expansion

$$
R\left(k^{2} \pm i 0 ; X_{l}\right)=E_{l 0}+k^{2} E_{l 2}+E_{l 3}(k), \quad k \rightarrow 0,
$$

in $B\left(L_{\text {com }}^{2}\left(I_{L}\right) \rightarrow L_{\text {loc }}^{2}\left(\mathbf{R}_{+}\right)\right)$.

(2) $E_{l 0}$ is formally self-adjoint and $\left\|\chi_{R} r^{-2} E_{l 0}\right\|=O\left(|l|^{-2}\right)$.

(3) $E_{l 2}$ is also formally self-adjoint and $\left\|\chi_{R} r^{-2} E_{l 2}\right\|=O\left(|l|^{-1}\right)$.

(4) $E_{l 3}(k)$ is of class $O p\left(o\left(k^{2}\right)\right)$ and $\left\|\chi_{R} r^{-2} E_{l 3}(k)\right\|=|l|^{-1} o\left(k^{2}\right)$.

4.3. We conclude the section by stating several important properties of $E_{l 0}$ and $E_{l 2}$ as a series of lemmas.

Lemma 4.3. Assume that $l=0$ or 1 . Let $f, g \in L_{\text {com }}^{2}\left(I_{L}\right)$ and let $u_{l}=$ $u_{l}(r)$ be as above. If $f$ satisfies $\left(f, u_{l}\right)=0$, then $\left(E_{l 2} f, g\right)=\left(E_{l 0} f, E_{l 0} g\right)$. 
Proof. We write $R_{l}( \pm k)$ for $R\left(k^{2} \pm i 0 ; X_{l}\right)$. If $f$ satisfies the assumption in the lemma, then $E_{l 0} f \in L_{\text {com }}^{2}\left(I_{L}\right)$ and $E_{l 1}( \pm k) f(r)=\gamma_{l}( \pm k)\left(f, u_{l}\right) u_{l}(r)$ $=0$. Thus we have

$$
\left(E_{l 2} f, g\right)=\lim _{k \rightarrow 0} k^{-2}\left(\left(R_{l}(-k)-E_{l 0}\right) f, g\right)=\lim _{k \rightarrow 0}\left(E_{l 0} f, R_{l}(k) g\right) .
$$

This proves the lemma.

Lemma 4.4. Assume that $l \neq 0,1$. Let $\operatorname{Im} \sqrt{\zeta}>0$ strictly and let $u \in L^{2}\left(\mathbf{R}_{+}\right)$be a solution to $X_{l} u-\zeta u=f$ with $f \in L_{\text {com }}^{2}\left(I_{L}\right)$. Then $u(r)$ obeys the bound

$$
u(r)=O\left(|\zeta|^{\nu / 2}\right) r^{1 / 2} H_{\nu}(\sqrt{\zeta} r), \quad|\zeta| \rightarrow 0,
$$

uniformly in $r \gg 1$.

If $|l|>l_{0}$, then

$$
u(r)=(i \pi / 2) r^{1 / 2} H_{\nu}(\sqrt{\zeta} r) \int_{0}^{\infty} p^{1 / 2} J_{\nu}(\sqrt{\zeta} p) f(p) d p
$$

for $r \gg 1$, and hence the lemma is obtained in the case $|l|>l_{0}$. When $|l| \leq l_{0}$ with $l \neq 0,1$, we will prove the lemma in section 9 . This is implicitly shown in the course of the proof of Proposition 4.1.

Lemma 4.5. Assume that $l \neq 0,1$. Let $f, g \in L_{\mathrm{com}}^{2}\left(I_{L}\right)$. Then

$$
\left(E_{l 2} f, g\right)=\left(E_{l 0} f, E_{l 0} g\right) .
$$

Proof. If $l \neq 0,1$, then both $E_{l 0} f$ and $E_{l 0} g$ belong to $L^{2}\left(\mathbf{R}_{+}\right)$, and hence the relation in the lemma makes sense. As previously stated, Propositions 4.1 and 4.2 remain true for $R\left(\zeta ; X_{l}\right)$ with $\operatorname{Im} \sqrt{\zeta}>0$. Hence we obtain

$$
\left(E_{l 2} f, g\right)=\lim _{\zeta \rightarrow 0}\left(E_{l 0} f, R\left(\zeta ; X_{l}\right) g\right)
$$

by use of the same argument as in the proof of Lemma 4.3. We divide the scalar product on the right side into the sum of three integrals

$$
\begin{aligned}
\int_{0}^{\infty} \cdots d r & =\left(\int_{0}^{R}+\int_{R}^{1 / k}+\int_{1 / k}^{\infty}\right) \cdots d r \\
& =I_{1}(k)+I_{2}(k)+I_{3}(k), \quad k=|\zeta|^{1 / 2},
\end{aligned}
$$


for $R \gg 1$ fixed. It is easy to see that

$$
\lim _{k \rightarrow 0} I_{1}(k)=\int_{0}^{R}\left(E_{l 0} f\right)(r) \overline{\left(E_{l 0} g\right)}(r) d r .
$$

We may assume that $f$ and $g$ have support in $[0, R)$. If $r>R$, then $E_{l 0} f$ and $R\left(\zeta ; X_{l}\right) g$ take the forms

$$
E_{l 0} f(r)=c_{0} r^{-\nu+1 / 2}, \quad R\left(\zeta ; X_{l}\right) g(r)=c(\zeta) r^{1 / 2} H_{\nu}(\sqrt{\zeta} r)
$$

for some constants $c_{0}$ and $c(\zeta)$. By Lemma 4.4, $c(\zeta)$ obeys the bound $|c(\zeta)|=$ $O\left(|k|^{\nu}\right)$ as $k=|\zeta|^{1 / 2} \rightarrow 0$, and $H_{\nu}(z)$ satisfies $H_{\nu}(z)=O\left(|z|^{-\nu}\right)$ as $|z| \rightarrow 0$. Thus we obtain

$$
\left|R\left(\zeta ; X_{l}\right) g(r)\right| \leq c r^{-\nu+1 / 2}, \quad R<r<1 / k,
$$

for $c>0$ independent of $k$. When $l \neq 0,1, \nu=|l-\alpha|>1$ and $r^{-2 \nu+1}$ is integrable over $[R, \infty)$. Hence it follows by the dominated convergence theorem that

$$
\lim _{k \rightarrow 0} I_{2}(k)=\int_{R}^{\infty}\left(E_{l 0} f\right)(r) \overline{\left(E_{l 0} g\right)}(r) d r .
$$

To deal with the third term $I_{3}(k)$, we use the asymptotic formula

$$
H_{\nu}(z)=(2 / \pi z)^{1 / 2} \exp (i(z-(2 \nu+1) \pi / 4))\left(1+O\left(|z|^{-1}\right)\right), \quad|z| \rightarrow \infty .
$$

A simple computation using integration by parts shows that $I_{3}(k)=$ $O\left(k^{2 \nu-2}\right)$ as $k \rightarrow 0$. Thus the proof is completed.

LEMmA 4.6. $E_{l 2}$ satisfies $X_{l} E_{l 2}=E_{l 0}$ on $L_{\mathrm{com}}^{2}\left(I_{L}\right)$.

Proof. Let $f \in L_{\text {com }}^{2}\left(I_{L}\right)$. We have only to show that $\left(E_{l 2} f, X_{l} g\right)=$ $\left(E_{l 0} f, g\right)$ for any $g \in C_{0}^{\infty}((0,2 L))$. If $l=0$ or 1 , then $\left(X_{l} g, u_{l}\right)=\left(g, X_{l} u_{l}\right)$ $=0$. Hence it follows from Lemma 4.3 that

$$
\left(E_{l 2} f, X_{l} g\right)=\left(E_{l 0} f, E_{l 0} X_{l} g\right)=\left(E_{l 0} f, g\right) .
$$

Lemma 4.5 enables us to repeat the same argument as above for the case $l \neq 0,1$ also. Thus the proof is complete.

LEMmA 4.7. Assume that $f \in L_{\text {com }}^{2}\left(I_{L}\right)$. Then one has:

(1) Let $l=0$ or 1 . Assume that $f$ satisfies $\left(f, u_{l}\right)=0$. Then $E_{l 0} f \in$ $L^{2}\left(\mathbf{R}_{+}\right)$and $\left\|E_{l 0} f\right\| \leq c\|f\|$. If, conversely, $E_{l 0} f \in L^{2}\left(\mathbf{R}_{+}\right)$, then $\left(f, u_{l}\right)$ $=0$.

(2) If $l \neq 0,1$, then $\left\|E_{l 0} f\right\| \leq c\|f\|$ uniformly in $l$. 
Proof. (1) can be easily verified. We prove (2) only for the case $|l| \gg 1$. By (4.6), $E_{l 0}(r, p)$ obeys $\left|E_{l 0}(r, p)\right| \leq L / 2 \nu$ for $(r, p) \in[0, L] \times[0, L]$. Hence

$$
\left|E_{l 0} f(r)\right| \leq \int_{0}^{r}\left|E_{l 0}(r, p) f(p)\right| d p=O(1)\|f\|, \quad|l| \rightarrow \infty,
$$

for $0<r \leq L$. On the other hand, if $r>L$, then

$$
\left|E_{l 0} f(r)\right| \leq(2 \nu)^{-1} r^{-\nu+1 / 2} \int_{0}^{L} p^{\nu+1 / 2}|f(p)| d p=O(1) L^{\nu+1} r^{-\nu+1 / 2}\|f\| .
$$

We combine these two estimates to conclude the proof.

\section{$\S 5$. Preliminaries for resolvent estimate II}

In this section we study the behavior as $\lambda \rightarrow 0$ of $R\left(\lambda+i 0 ; K_{\alpha}\right)$ for the operator $K_{\alpha}$ defined by (4.1).

5.1. We begin with the following proposition on the uniqueness of solution to equation $K_{\alpha} w=0$.

Proposition 5.1. Let $w \in L_{\mathrm{loc}}^{2}\left(\mathbf{R}^{2}\right)$ be a solution to $K_{\alpha} w=0$. If $w$ satisfies

$$
\limsup _{R \rightarrow \infty} R^{-2} \int_{R<|x|<2 R}|w(x)|^{2} d x<\infty,
$$

then $w=0$.

Remark. The proposition above remains true for magnetic Schrödinger operators with fields not indentically vanishing.

Proof. The proposition is easy to prove. We write $K_{\alpha}$ in the form $K_{\alpha}=\Pi_{1}^{2}+\Pi_{2}^{2}$, so that the magnetic field $b_{\alpha}$ of $K_{\alpha}$ is expressed as the commutator $b_{\alpha}=i\left[\Pi_{2}, \Pi_{1}\right]$. We note that $b_{\alpha}$ does not indentially vanish. Let $\chi_{R}(x)=\chi(|x| / R)$. Then we have

$$
\left(K_{\alpha} w, \chi_{R} w\right)=\sum_{j=1}^{2}\left(\chi_{R} \Pi_{j} w, \Pi_{j} w\right)-\left(\left(\Delta \chi_{R}\right) w, w\right),
$$

where ( , ) denotes the $L^{2}$ scalar product in $L^{2}\left(\mathbf{R}^{2}\right)$. By assumption, the second term on the right side is bounded uniformly in $R \gg 1$ and hence it follows that $\Pi_{j} w \in L^{2}\left(\mathbf{R}^{2}\right)$. This also implies that

$$
\sum_{j=1}^{2}\left(\Pi_{j} w, \Pi_{j} w\right)=\left(K_{\alpha} w, w\right)=0,
$$


so that $\Pi_{j} w=0$. Since $b_{\alpha} w=i\left[\Pi_{2}, \Pi_{1}\right] w=0, w$ vanishes on the support of the field $b_{\alpha}$. This, together with relation $\Pi_{j} w=0$, proves the proposition.

If $w(x)$ is bounded or if it belongs to the weighted $L^{2}$ space $L_{-1}^{2}\left(\mathbf{R}^{2}\right)$, then the assumption in Proposition 5.1 is fulfilled. The proposition is used for such solutions in the later application. As an immediate consequence of the above proposition, we obtain the following lemma.

LEMMA 5.1. Let $K_{l \alpha}$ be defined by (4.2) and let $w_{l} \in L_{\text {loc }}^{2}\left(\mathbf{R}_{+}\right)$be a solution to $K_{l \alpha} w_{l}=0$. If $w_{l}$ satisfies

$$
\limsup _{R \rightarrow \infty} R^{-2} \int_{R}^{2 R}\left|w_{l}(r)\right|^{2} d r<\infty
$$

and the boundary condition $\lim _{r \rightarrow 0} r^{-1 / 2} w_{l}(r)<\infty$, then $w_{l}=0$.

We now set $W_{l}=K_{l \alpha}-X_{l}$. Then $W_{l}$ takes the form

$$
W_{l}(r)= \begin{cases}\left(l-\alpha \chi_{\infty}(r)\right)^{2} r^{-2}-q_{l}(r) & \text { for }|l| \leq l_{0}, \\ \left(\left(l-\alpha \chi_{\infty}(r)\right)^{2}-\nu^{2}\right) r^{-2} & \text { for }|l|>l_{0} .\end{cases}
$$

By definition, $W_{l}(r)$ vanishes in $r>2$ for all $l \in \mathbf{Z}$. If $|l| \leq l_{0}$, then $W_{l}(r)$ is bounded and if $|l|>l_{0}$, then $W_{l}(r)$ obeys the bound $\left|W_{l}(r)\right| \leq c|l| r^{-2}$ uniformly in $l$.

LEMMA 5.2. If we regard $\mathrm{Id}+W_{l} E_{l 0}$ as an operator from $L_{\mathrm{com}}^{2}\left(I_{L}\right)$ into itself, then it has an inverse for all $l \in \mathbf{Z}$ and the inverse

$$
T_{l}=\left(\mathrm{Id}+W_{l} E_{l 0}\right)^{-1}: L_{\mathrm{com}}^{2}\left(I_{L}\right) \longrightarrow L_{\mathrm{com}}^{2}\left(I_{L}\right)
$$

is bounded uniformly in $l$.

Proof. If $|l|>l_{0}$, then the lemma follows from Lemma 4.1 at once. We consider the case $|l| \leq l_{0}$. Since $W_{l} E_{l 0}: L_{\text {com }}^{2}\left(I_{L}\right) \rightarrow L_{\text {com }}^{2}\left(I_{L}\right)$ is a compact operator, it suffices to show that

$$
w \in L_{\text {com }}^{2}\left(I_{L}\right), \quad w+W_{l} E_{l 0} w=0 \Longrightarrow w=0 .
$$

To see this, we set $v=E_{l 0} w$. Then $v$ satisfies $X_{l} v=w$, so that $K_{l \alpha} v=$ $X_{l} v+W_{l} v=0$. As is easily seen, $v$ behaves like $v(r) \sim r^{-\nu+1 / 2}$ at infinity and it satisfies the assumptions in Lemma 5.1. Thus we can conclude that $v=0$ and hence $w=0$. 
By Proposition 4.1, $E_{l 1}( \pm k): L_{\mathrm{com}}^{2}\left(I_{L}\right) \rightarrow L_{\mathrm{loc}}^{2}\left(\mathbf{R}_{+}\right)$is of class $O p\left(O\left(k^{2 \nu}\right)\right)$. Hence we can define

$$
T_{l}( \pm k)=\left(\mathrm{Id}+T_{l} W_{l} E_{l 1}( \pm k)\right)^{-1} T_{l}: L_{\mathrm{com}}^{2}\left(I_{L}\right) \longrightarrow L_{\mathrm{com}}^{2}\left(I_{L}\right)
$$

for $l=0,1$. By definition, $T_{l}( \pm k)$ is expanded as

$$
T_{l}( \pm k)=\sum_{j=0}^{\infty}(-1)^{j}\left(T_{l} W_{l} E_{l 1}( \pm k)\right)^{j} T_{l}
$$

and hence

$$
E_{l 1}( \pm k) T_{l}( \pm k) f=0, \quad f \in L_{\text {com }}^{2}\left(I_{L}\right),
$$

provided that $\left(T_{l} f, u_{l}\right)=0$. We also have the relation

$$
T_{l}( \pm k)=T_{l}-T_{l} W_{l} E_{l 1}( \pm k) T_{l}( \pm k)=T_{l}+O p\left(O\left(k^{2 \nu}\right)\right)
$$

in $B\left(L_{\mathrm{com}}^{2}\left(I_{L}\right) \rightarrow L_{\mathrm{com}}^{2}\left(I_{L}\right)\right)$.

5.2. We first study the behavior as $k \rightarrow 0$ of $R\left(k^{2}+i 0 ; K_{l \alpha}\right)$. By the resolvent identity,

$$
R\left(k^{2}+i 0 ; K_{l \alpha}\right)=R\left(k^{2}+i 0 ; X_{l}\right)\left(\operatorname{Id}+W_{l} R\left(k^{2}+i 0 ; X_{l}\right)\right)^{-1} .
$$

Hence Lemma 5.2 implies that there exists a limit

$$
F_{l 0}=\lim _{k \rightarrow 0} R\left(k^{2}+i 0 ; K_{l \alpha}\right)=E_{l 0} T_{l}: L_{\mathrm{com}}^{2}\left(I_{L}\right) \longrightarrow L_{\mathrm{loc}}^{2}\left(\mathbf{R}_{+}\right)
$$

and that it is bounded uniformly in $l$ as an operator in $B\left(L_{\text {com }}^{2}\left(I_{L}\right) \rightarrow\right.$ $\left.L_{\text {loc }}^{2}\left(\mathbf{R}_{+}\right)\right)$. We can easily show that

$$
T_{l}=\left(\mathrm{Id}+W_{l} E_{l 0}\right)^{-1}=\mathrm{Id}-W_{l} F_{l 0}: L_{\mathrm{com}}^{2}\left(I_{L}\right) \longrightarrow L_{\mathrm{com}}^{2}\left(I_{L}\right) .
$$

Hence the formal adjoint of $T_{l}$ is defined by

$$
T_{l}^{*}=\mathrm{Id}-F_{l 0} W_{l}: L_{\mathrm{loc}}^{2}\left(\mathbf{R}_{+}\right) \longrightarrow L_{\mathrm{loc}}^{2}\left(\mathbf{R}_{+}\right) .
$$

If $|l| \leq l_{0}$, then $W_{l}$ is bounded and hence we have

$$
\left(T_{l} W_{l}\right)^{*}=T_{l} W_{l} .
$$


Even for $|l|>l_{0}$, we can define

$$
T_{l}^{*} E_{l 2} T_{l}=\left(\mathrm{Id}-F_{l 0} W_{l}\right) E_{l 2} T_{l}: L_{\mathrm{com}}^{2}\left(I_{L}\right) \longrightarrow L_{\mathrm{loc}}^{2}\left(\mathbf{R}_{+}\right)
$$

as an operator bounded uniformly in $l$, which follows from Lemma 4.2.

We now expand the inverse

$$
\left(\mathrm{Id}+W_{l} R\left(k^{2}+i 0 ; X_{l}\right)\right)^{-1}: L_{\mathrm{com}}^{2}\left(I_{L}\right) \longrightarrow L_{\mathrm{com}}^{2}\left(I_{L}\right)
$$

in $B\left(L_{\text {com }}^{2}\left(I_{L}\right) \rightarrow L_{\text {com }}^{2}\left(I_{L}\right)\right)$. By Propositions 4.1 and 4.2, we can show that: If $l=0$ or 1 , then

$$
\left(\mathrm{Id}+W_{l} R\left(k^{2}+i 0 ; X_{l}\right)\right)^{-1}=T_{l}(k)-k^{2} T_{l} W_{l} E_{l 2} T_{l}+O p\left(o\left(k^{2}\right)\right)
$$

and if $l \neq 0,1$, then

$$
\left(\mathrm{Id}+W_{l} R\left(k^{2}+i 0 ; X_{l}\right)\right)^{-1}=T_{l}-k^{2} T_{l} W_{l} E_{l 2} T_{l}+O p\left(o\left(k^{2}\right)\right)
$$

uniformly in $|l| \gg 1$. A similar expansion remains true for $R\left(k^{2}-i 0 ; X_{l}\right)$.

LEMMA 5.3. (1) The resolvent $R\left(k^{2} \pm i 0 ; K_{l \alpha}\right)$ has the following expansion in $B\left(L_{\mathrm{com}}^{2}\left(I_{L}\right) \rightarrow L_{\mathrm{loc}}^{2}\left(\mathbf{R}_{+}\right)\right)$: If $l=0$ or 1 , then

$$
R\left(k^{2} \pm i 0 ; K_{l \alpha}\right)=F_{l 0}+F_{l 1}( \pm k)+k^{2} F_{l 2}+O p\left(o\left(k^{2}\right)\right)
$$

and if $l \neq 0,1$, then

$$
R\left(k^{2} \pm i 0 ; K_{l \alpha}\right)=F_{l 0}+k^{2} F_{l 2}+O p\left(o\left(k^{2}\right)\right)
$$

uniformly in $|l| \gg 1$, where

$$
F_{l 0}=E_{l 0} T_{l}, \quad F_{l 1}(k)=T_{l}^{*} E_{l 1}(k) T_{l}(k), \quad F_{l 2}=T_{l}^{*} E_{l 2} T_{l} .
$$

(2) $F_{l 0}$ and $F_{l 2}$ are formally self-adjoint and are bounded uniformly in $|l| \gg 1$ as operators in $B\left(L_{\mathrm{com}}^{2}\left(I_{L}\right) \rightarrow L_{\mathrm{loc}}^{2}\left(\mathbf{R}_{+}\right)\right)$.

(3) $F_{l 1}(k), l=0,1$, belongs to $O p\left(O\left(k^{2 \nu}\right)\right)$ and satisfies $F_{l 1}^{*}(k)=F_{l 1}(-k)$. This acts as

$$
F_{l 1}(k) f(r)=\gamma_{l}(k)\left(T_{l}(k) f, u_{l}\right)_{L^{2}\left(\mathbf{R}_{+}\right)}\left(T_{l}^{*} u_{l}\right)(r)
$$

for $f \in L_{\mathrm{com}}^{2}\left(I_{L}\right), \gamma_{l}(k)$ being as in Proposition 4.1 . 
Proof. (1) If $l=0,1$, then it follows from (5.5) and (5.8) that

$$
R\left(k^{2}+i 0 ; K_{l \alpha}\right)=F_{l 0}+F_{l 1}(k)+k^{2} F_{l 2}+O p\left(o\left(k^{2}\right)\right),
$$

where $F_{l 0}=E_{l 0} T_{l}$, and

$$
\begin{aligned}
F_{l 1}(k) & =E_{l 0}\left(T_{l}(k)-T_{l}\right)+E_{l 1}(k) T_{l}(k), \\
F_{l 2} & =-E_{l 0} T_{l} W_{l} E_{l 2} T_{l}+E_{l 2} T_{l} .
\end{aligned}
$$

If we further make use of relations (5.4) and (5.6), then we have

$$
\begin{aligned}
F_{l 1}(k) & =\left(\operatorname{Id}-E_{l 0} T_{l} W_{l}\right) E_{l 1}(k) T_{l}(k) \\
& =\left(\operatorname{Id}-F_{l 0} W_{l}\right) E_{l 1}(k) T_{l}(k) \\
& =T_{l}^{*} E_{l 1}(k) T_{l}(k) .
\end{aligned}
$$

We can show in the same way that $F_{l 2}=T_{l}^{*} E_{l 2} T_{l}$. A similar argument applies to the case $l \neq 0,1$ and we can obtain the asymptotic expansion with remainder operator $O p\left(o\left(k^{2}\right)\right)$ bounded uniformly in $|l| \gg 1$. Thus (1) is verified. (2) As already stated, $F_{l 0}$ and $F_{l 2}$ are uniformly bounded in $B\left(L_{\mathrm{com}}^{2}\left(I_{L}\right) \rightarrow L_{\mathrm{loc}}^{2}\left(\mathbf{R}_{+}\right)\right)$, and it is easy to see that these operators are formally self-adjoint. (3) Since $E_{l 1}(k) \in O p\left(O\left(k^{2 \nu}\right)\right)$ satisfies $E_{l 1}^{*}(k)=$ $E_{l 1}(-k), F_{l 1}(k)$ also belongs to $\in O p\left(O\left(k^{2 \nu}\right)\right)$, and it follows from (5.7) that

$$
\begin{aligned}
F_{l 1}^{*}(k) & =T_{l}^{*}(k) E_{l 1}(-k) T_{l}=T_{l}^{*}\left(\sum_{j=0}^{\infty}(-1)^{j}\left(E_{l 1}(-k) T_{l} W_{l}\right)^{j}\right) E_{l 1}(-k) T_{l} \\
& =T_{l}^{*} E_{l 1}(-k)\left(\sum_{j=0}^{\infty}(-1)^{j}\left(T_{l} W_{l} E_{l 1}(-k)\right)^{j}\right) T_{l}=F_{l 1}(-k) .
\end{aligned}
$$

Thus $F_{l 1}(k)$ preserves the same properties as $E_{l 1}(k)$. It can be easily seen from Proposition 4.1 that $F_{l 1}(k)$ acts as in the lemma.

The operators $F_{l 0}$ and $F_{l 2}$ have the same properties as $E_{l 0}$ and $E_{l 2}$.

Lemma 5.4. Let $f, g \in L_{\mathrm{com}}^{2}\left(I_{L}\right)$. Then one has:

(1) Assume that $l=0$ or 1. If $\left(T_{l} f, u_{l}\right)=0$, then $\left(F_{l 2} f, g\right)=\left(F_{l 0} f, F_{l 0} g\right)$.

(2) Assume that $l \neq 0,1$. Then $\left(F_{l 2} f, g\right)=\left(F_{l 0} f, F_{l 0} g\right)$. 
Proof. (1) If $f$ satisfies the assumption in the lemma, then $F_{l 0} f=$ $E_{l 0} T_{l} f \in L_{\text {com }}^{2}\left(I_{L}\right)$ and it follows from (5.3) that $F_{l 1}(k) f=T_{l}^{*} E_{l 1}(k) T_{l}(k) f$ $=0$. This enables us to repeat the same argument as in the proof of Lemma 4.3 and we get the desired relation. (2) Since $T_{l} f \in L_{\text {com }}^{2}\left(I_{L}\right)$ for $f \in L_{\text {com }}^{2}\left(I_{L}\right)$, this is an immediate consequence of Lemma 4.5. In fact, we have

$$
\left(F_{l 2} f, g\right)=\left(E_{l 2} T_{l} f, T_{l} g\right)=\left(E_{l 0} T_{l} f, E_{l 0} T_{l} g\right)=\left(F_{l 0} f, F_{l 0} g\right) .
$$

Thus (2) is verified.

LEMMA 5.5. $\quad F_{l 2}$ satisfies $K_{l \alpha} F_{l 2}=F_{l 0}$ on $L_{\mathrm{com}}^{2}\left(I_{L}\right)$.

Proof. By (5.6), $u_{l}(r), l=0,1$, satisfies

$$
K_{l \alpha} T_{l}^{*} u_{l}=K_{l \alpha}\left(u_{l}-F_{l 0} W_{l} u_{l}\right)=X_{l} u_{l}=0 .
$$

This yields that $\left(T_{l} K_{l \alpha} g, u_{l}\right)=0$ for $g \in C_{0}^{\infty}((0,2 L))$. Hence the lemma is proved in exactly the same way as in the proof of Lemma 4.6, if we make use of Lemma 5.4 .

The next lemma is obtained from Lemma 4.7. We skip the proof.

Lemma 5.6. Assume that $f \in L_{\text {com }}^{2}\left(I_{L}\right)$. Then one has:

(1) Assume that $l=0$ or 1 . If $f$ satisfies $\left(T_{l} f, u_{l}\right)=0$, then $F_{l 0} f \in$ $L^{2}\left(\mathbf{R}_{+}^{2}\right)$ and $\left\|F_{l 0} f\right\| \leq c\|f\|$. If, conversely, $F_{l 0} f \in L^{2}\left(\mathbf{R}_{+}^{2}\right)$, then $\left(T_{l} f, u_{l}\right)$ $=0$.

(2) If $l \neq 0,1$, then $\left\|F_{l 0} f\right\| \leq c\|f\|$ uniformly in $l$.

5.3. We proceed to the study on the behavior at low energy of $R(\lambda+$ $\left.i 0 ; K_{\alpha}\right)$. We use similar notations $L_{\text {com }}^{2}\left(\Sigma_{L}\right), B\left(L_{\text {com }}^{2}\left(\Sigma_{L}\right) \rightarrow L_{\text {loc }}^{2}\left(\mathbf{R}^{2}\right)\right)$ and $O p\left(O\left(k^{\gamma}\right)\right)$ with natural modifications for the domain $\Sigma_{L}=\left\{x \in \mathbf{R}^{2}\right.$ : $|x|<2 L\}$ with $L \gg 1$ fixed again. Let $U_{l}, l \in \mathbf{Z}$, be defined by

$$
U_{l} f(r)=(2 \pi)^{-1 / 2} \int_{0}^{2 \pi} r^{1 / 2} f(r \theta) e^{-i l \theta} d \theta: L_{\mathrm{loc}}^{2}\left(\mathbf{R}^{2}\right) \longrightarrow L_{\mathrm{loc}}^{2}\left(\mathbf{R}_{+}\right)
$$

If $f, g \in L^{2}\left(\mathbf{R}^{2}\right)$, then

$$
(f, g)_{L^{2}\left(\mathbf{R}^{2}\right)}=\sum_{l \in \mathbf{Z}}\left(U_{l} f, U_{l} g\right)_{L^{2}\left(\mathbf{R}_{+}\right)}
$$


and the formal adjoint operator $U_{l}^{*}$ is calculated as

$$
U_{l}^{*} g(x)=(2 \pi)^{-1 / 2} r^{-1 / 2} g(r) e^{i l \theta}: L_{\mathrm{loc}}^{2}\left(\mathbf{R}_{+}\right) \longrightarrow L_{\mathrm{loc}}^{2}\left(\mathbf{R}^{2}\right) .
$$

We now define the following operators:

$$
\begin{aligned}
G_{0} & =\sum_{l \in \mathbf{Z}} U_{l}^{*} F_{l 0} U_{l}=\sum_{l \in \mathbf{Z}} U_{l}^{*} E_{l 0} T_{l} U_{l} \\
G_{2} & =\sum_{l \in \mathbf{Z}} U_{l}^{*} F_{l 2} U_{l}=\sum_{l \in \mathbf{Z}} U_{l}^{*} T_{l}^{*} E_{l 2} T_{l} U_{l}
\end{aligned}
$$

and $G_{1}( \pm k)=Z_{0}( \pm k)+Z_{1}( \pm k)$, where

$$
Z_{l}( \pm k)=U_{l}^{*} F_{l 1}( \pm k) U_{l}=U_{l}^{*} T_{l}^{*} E_{l 1}( \pm k) T_{l}( \pm k) U_{l} .
$$

We further define

$$
\tilde{u}_{l}(x)=\left(U_{l}^{*} T_{l}^{*} u_{l}\right)(x), \quad l=0,1
$$

By (5.9), $\tilde{u}_{l}(x)$ solves the equation $K_{\alpha} \tilde{u}_{l}=U_{l}^{*} K_{l \alpha} T_{l}^{*} u_{l}=0$. The next lemma is obtained as an immediate consequence of Lemma 5.3.

LEMMA 5.7. (1) $R\left(k^{2} \pm i 0 ; K_{\alpha}\right)$ has the asymptotic expansion

$$
R\left(k^{2} \pm i 0 ; K_{\alpha}\right)=G_{0}+G_{1}( \pm k)+k^{2} G_{2}+O p\left(o\left(k^{2}\right)\right)
$$

in $B\left(L_{\mathrm{com}}^{2}\left(\Sigma_{L}\right) \rightarrow L_{\mathrm{loc}}^{2}\left(\mathbf{R}^{2}\right)\right)$.

(2) $G_{0}$ and $G_{2}$ are formally self-adjoint.

(3) $Z_{l}(k), l=0,1$, belongs to $O p\left(O\left(k^{2 \nu}\right)\right)$ as an operator in $B\left(L_{\mathrm{com}}^{2}\left(\Sigma_{L}\right)\right.$ $\left.\rightarrow L_{\mathrm{loc}}^{2}\left(\mathbf{R}^{2}\right)\right)$. It satisfies $Z_{l}^{*}(k)=Z_{l}(-k)$ and acts as

$$
Z_{l}(k) f(x)=\gamma_{l}(k)\left(T_{l}(k) U_{l} f, u_{l}\right)_{L^{2}\left(\mathbf{R}_{+}\right)} \tilde{u}_{l}(x)
$$

for $f \in L_{\mathrm{com}}^{2}\left(\Sigma_{L}\right), \gamma_{l}(k)$ being again as in Proposition 4.1 .

The following two lemmas also follow from Lemmas 5.4 and 5.6 at once.

LEMMA 5.8. Let $\tilde{u}_{l}$ be as above. Assume that $f, g \in L_{\mathrm{com}}^{2}\left(\Sigma_{L}\right)$. If $f$ satisfies $\left(f, \tilde{u}_{l}\right)=0$ (and hence $Z_{l}(k) f=0$ by $\left.(5.3)\right)$, then $\left(G_{2} f, g\right)=$ $\left(G_{0} f, G_{0} g\right)$. 
Lemma 5.9. Assume that $f \in L_{\mathrm{com}}^{2}\left(\Sigma_{L}\right)$. Then $G_{0} f$ takes the form

$$
G_{0} f=\sum_{l=0}^{1} U_{l}^{*} F_{l 0} U_{l} f+g, \quad g \in L^{2}\left(\mathbf{R}^{2}\right) .
$$

If $f$ satisfies $\left(f, \tilde{u}_{l}\right)=0$ for $l=0,1$, then $G_{0} f \in L^{2}\left(\mathbf{R}^{2}\right)$ and obeys $\left\|G_{0} f\right\| \leq c\|f\|$ for $c>0$ independent of $f$. If, conversely, $G_{0} f \in L^{2}\left(\mathbf{R}^{2}\right)$, then $\left(f, \tilde{u}_{l}\right)=0$.

We conclude the section by proving the following lemma.

LemMa 5.10. (1) $\nabla G_{0}$ and $\nabla G_{2}$ belong to $B\left(L_{\text {com }}^{2}\left(\Sigma_{L}\right) \rightarrow L_{\text {loc }}^{2}\left(\mathbf{R}^{2}\right)\right)$, and $\nabla Z_{l}( \pm k)$ is of class $O p\left(O\left(k^{2 \nu}\right)\right)$.

(2) $\nabla G( \pm k) \in O p\left(o\left(k^{2}\right)\right)$, where

$$
G( \pm k)=R\left(k^{2} \pm i 0 ; K_{\alpha}\right)-G_{0}-G_{1}( \pm k)-k^{2} G_{2} .
$$

Proof. The lemma follows by elliptic estimate. We prove (2) only. We first note that $K_{l \alpha} F_{l 0}=\mathrm{Id}$ on $L_{\text {com }}^{2}\left(\Sigma_{L}\right)$. By Lemma $5.5, K_{l \alpha} F_{l 2}=F_{l 0}$, and by $(5.9), K_{l \alpha} F_{l 1}(k)=0$. These relations yield

$$
K_{\alpha} G_{0}=\mathrm{Id}, \quad K_{\alpha} G_{1}(k)=0, \quad K_{\alpha} G_{2}=G_{0}
$$

on $L_{\text {com }}^{2}\left(\Sigma_{L}\right)$. Hence $G(k)$ obeys

$$
\left(K_{\alpha}-k^{2}\right) G(k)=k^{2} G_{1}(k)+k^{4} G_{2} .
$$

Since $G(k) \in O p\left(o\left(k^{2}\right)\right)$ by Lemma 5.7, (2) is obtained by elliptic estimate.

\section{$\S 6$. Resolvent estimate at low energy}

In this section we study the behavior as $\lambda \rightarrow 0$ of $R(\lambda+i 0 ; H)$. Recall that $W=H-K_{\alpha}, W^{*}=W$, is a first order differential operator having smooth coefficients with support in $\left\{x \in \mathbf{R}^{2}:|x|<2\right\}$. We may assume that $L \gg 1$ is chosen so large that

$$
\chi_{L} W=W \chi_{L}=W, \quad \chi_{L}(x)=\chi(|x| / L) .
$$

By the resolvent identity, we have

$$
\chi_{L} R\left(k^{2}+i 0 ; H\right) \chi_{L}=\chi_{L} R\left(k^{2}+i 0 ; K_{\alpha}\right)\left(\mathrm{Id}-W \chi_{L} R\left(k^{2}+i 0 ; H\right)\right) \chi_{L}
$$


and hence

$$
\chi_{L} R\left(k^{2}+i 0 ; H\right) \chi_{L}=\left(\mathrm{Id}+\chi_{L} R\left(k^{2}+i 0 ; K_{\alpha}\right) W\right)^{-1} \chi_{L} R\left(k^{2}+i 0 ; K_{\alpha}\right) \chi_{L},
$$

provided that the inverse

$$
\left(\mathrm{Id}+\chi_{L} R\left(k^{2}+i 0 ; K_{\alpha}\right) W\right)^{-1}: L^{2}\left(\mathbf{R}^{2}\right) \longrightarrow L^{2}\left(\mathbf{R}^{2}\right)
$$

exists. Thus the problem is reduced to analysing the behavior as $k \rightarrow 0$ of the inverse on the right side. To do this, we follow the idea due to JensenKato [7], although technical details are different in many aspects. We often use the following proposition in the argument below.

Proposition 6.1. Let $\mathcal{X}_{j}, \mathcal{Y}_{j}, j=0,1$, be vector spaces and let $A$ : $\mathcal{X}_{1} \rightarrow \mathcal{Y}_{1}$. Assume that $B: \mathcal{X}_{0} \rightarrow \mathcal{X}_{1}$ is surjective and $C: \mathcal{Y}_{1} \rightarrow \mathcal{Y}_{0}$ is injective. Define $\mathcal{A}=C A B: \mathcal{X}_{0} \rightarrow \mathcal{Y}_{0}$. If $\mathcal{A}^{-1}$ exists, then $A^{-1}=B \mathcal{A}^{-1} C$.

Proof. The lemma is due to [7, Lemma 3.12]. We repeat the argument there. Let $D=B \mathcal{A}^{-1} C: \mathcal{Y}_{1} \rightarrow \mathcal{X}_{1}$. Then

$$
C A D=C A B \mathcal{A}^{-1} C=\mathcal{A} \mathcal{A}^{-1} C=C .
$$

Since $C$ is injective, it follows that $A D=\mathrm{Id}$. On the other hand, we have

$$
D A B=B \mathcal{A}^{-1} C A B=B \mathcal{A}^{-1} \mathcal{A}=B
$$

Since $B$ is surjective, we obtain $D A=\mathrm{Id}$. This proves the proposition.

6.1. Let $G_{0}=K_{\alpha}^{-1}$ be as in Lemma 5.7. We define the subspace $\mathcal{M}$ of $L^{2}\left(\mathbf{R}^{2}\right)$ by

$$
\mathcal{M}=\left\{u \in L^{2}\left(\mathbf{R}^{2}\right):\left(\operatorname{Id}+\chi_{L} G_{0} W\right) u=0\right\} .
$$

Since $\chi_{L} G_{0} W: L^{2}\left(\mathbf{R}^{2}\right) \rightarrow L^{2}\left(\mathbf{R}^{2}\right)$ is compact, the dimension of the space $\mathcal{M}$ is finite $\operatorname{dim} \mathcal{M}<\infty$. We further introduce another subspace of $L_{-1}^{2}\left(\mathbf{R}^{2}\right)$. Since $G_{0} W: L_{-1}^{2}\left(\mathbf{R}^{2}\right) \rightarrow L_{-1}^{2}\left(\mathbf{R}^{2}\right)$ is well defined, we can define

$$
\mathcal{E}=\left\{u \in L_{-1}^{2}\left(\mathbf{R}^{2}\right):\left(\operatorname{Id}+G_{0} W\right) u=0\right\} .
$$

LEMMA 6.1. If $v=G_{0} W u$ for $u \in C^{\infty}\left(\mathbf{R}^{2}\right)$, then $G_{0} K_{\alpha} v=v$. 
Proof. By assumption, $K_{\alpha} v=W u \in C_{0}^{\infty}\left(\mathbf{R}^{2}\right)$. We set $w=G_{0} K_{\alpha} v-v$. Then $w$ belongs to $L_{-1}^{2}\left(\mathbf{R}^{2}\right)$ and it solves the equation $K_{\alpha} w=K_{\alpha}\left(G_{0} K_{\alpha} v-\right.$ $v)=0$. However such a solution identically vanishes by Proposition 5.1. This proves the lemma.

LEMMA 6.2.

(1) $G_{0} W: \mathcal{M} \rightarrow \mathcal{E}$ is injective and surjective.

(2) $\chi_{L}: \mathcal{E} \rightarrow \mathcal{M}$ is also injective and surjective.

Proof. (1) Let $u \in \mathcal{M}$ and set $v=G_{0} W u$. We assert that $v \in \mathcal{E}$. If $u \in \mathcal{M}$, then $u \in C_{0}^{\infty}\left(\mathbf{R}^{2}\right)$ by the regularity property for solutions to elliptic equations. Since $v$ satisfies $K_{\alpha} v=W u \in C_{0}^{\infty}\left(\mathbf{R}^{2}\right)$, we have $K_{\alpha} v+W v=$ $W\left(u+\chi_{L} G_{0} W u\right)=0$ by (6.1). Hence it follows from Lemma 6.1 that

$$
v+G_{0} W v=G_{0}\left(K_{\alpha} v+W v\right)=0 .
$$

This proves that $v \in \mathcal{E}$. If $G_{0} W u=0$ for $u \in \mathcal{M}$, then $u=-\chi_{L} G_{0} W u=$ 0 and hence $G_{0} W$ is shown to be injective. We shall show that $G_{0} W$ is surjective. Let $v \in \mathcal{E}$. We set $u=-\chi_{L} v$. Then $u$ belongs to $L^{2}\left(\mathbf{R}^{2}\right)$ and satisfies

$$
\left(\mathrm{Id}+\chi_{L} G_{0} W\right) u=-\left(\operatorname{Id}+\chi_{L} G_{0} W\right) \chi_{L} v=-\chi_{L}\left(v+G_{0} W v\right)=0 .
$$

Hence $u \in \mathcal{M}$ and we have

$$
v=-G_{0} W v=-G_{0} W \chi_{L} v=G_{0} W u
$$

This proves that $G_{0} W$ is surjective. (2) By (1), $\operatorname{dim} \mathcal{E}=\operatorname{dim} \mathcal{M}<\infty$. We have already shown that $\chi_{L}: \mathcal{E} \rightarrow \mathcal{M}$ is well defined. It is easy to see that $\chi_{L}$ is injective. This proves (2).

Let $\tilde{u}_{l}$ be defined by (5.10). We further define the following two subspaces:

$$
\mathcal{M}_{0}=\left\{u \in \mathcal{M}:\left(u, W \tilde{u}_{l}\right)=0, l=0,1\right\}, \quad \mathcal{E}_{0}=\left\{u \in \mathcal{E}: u \in L^{2}\left(\mathbf{R}^{2}\right)\right\}
$$

Obviously $u \in \mathcal{E}_{0}$ means that $u$ is a bound state of $H$ associated with zero eigenvalue.

LEMMA 6.3.

(1) $G_{0} W: \mathcal{M}_{0} \rightarrow \mathcal{E}_{0}$ is injective and surjective.

(2) $\chi_{L}: \mathcal{E}_{0} \rightarrow \mathcal{M}_{0}$ is also injective and surjective. 
Proof. (1) We set $v=G_{0} W u$ for $u \in \mathcal{M}_{0}$. Since $\left(W u, \tilde{u}_{l}\right)=\left(u, W \tilde{u}_{l}\right)$ $=0$, it follows from Lemma 5.9 that $v \in L^{2}\left(\mathbf{R}^{2}\right)$, and $v$ obeys $H v=0$. Thus $G_{0} W: \mathcal{M}_{0} \rightarrow \mathcal{E}_{0}$ is well defined. It is easy to see that $G_{0} W$ is injective. To prove that it is surjective, we again set $u=-\chi_{L} v$ for $v \in \mathcal{E}_{0}$. Then $v$ is represented as $v=G_{0} W u \in L^{2}\left(\mathbf{R}^{2}\right)$. Hence it follows again from Lemma 5.9 that $\left(W u, \tilde{u}_{l}\right)=0$ for $l=0,1$. This implies that $u \in \mathcal{M}_{0}$ and $G_{0} W$ is shown to be surjective. (2) This is verified in the same way as used to prove Lemma $6.2(2)$.

LEMMA 6.4. The geometric null space $\mathcal{M}$ coincides with the algebraic null space of the operator $\operatorname{Id}+\chi_{L} G_{0} W$ acting on $L^{2}\left(\mathbf{R}^{2}\right)$.

Proof. To prove the lemma, it suffices to show that: If $u=(\mathrm{Id}+$ $\left.\chi_{L} G_{0} W\right) u_{1} \in \mathcal{M}$ for some $u_{1} \in L^{2}\left(\mathbf{R}^{2}\right)$, then $u=0$. To see this, we calculate

$$
(u, W u)=\left(\left(\operatorname{Id}+\chi_{L} G_{0} W\right) u_{1}, W u\right)=\left(u_{1}, W\left(\operatorname{Id}+\chi_{L} G_{0} W\right) u\right)=0 .
$$

Since $u \in \mathcal{M}$ satisfies $W u=-W G_{0} W u$, we have $\left(W u, G_{0} W u\right)=0$. Set $v=$ $G_{0} W u \in L_{-1}^{2}\left(\mathbf{R}^{2}\right)$. Then $K_{\alpha} v=W u$ and hence $\left(K_{\alpha} v, v\right)=0$. This enables us to repeat the the same argument as in the proof of Proposition 5.1. We can show that $v=0$, which implies that $u=-\chi_{L} G_{0} W u=-\chi_{L} v=0$. Thus the proof is complete.

As a consequence of Lemma 6.4, we obtain that: (1) There exists a projection (not necessarily orthogonal) $Q: L^{2}\left(\mathbf{R}^{2}\right) \rightarrow \mathcal{M}, Q^{2}=Q$, on the space $\mathcal{M}$. (2) There exists a bounded operator $Y_{3}: L^{2}\left(\mathbf{R}^{2}\right) \rightarrow L^{2}\left(\mathbf{R}^{2}\right)$ such that

$$
\left(\mathrm{Id}+\chi_{L} G_{0} W\right) Y_{3}=Y_{3}\left(\mathrm{Id}+\chi_{L} G_{0} W\right)=\mathrm{Id}-Q, \quad Q Y_{3}=Y_{3} Q=0 .
$$

Let

$$
P_{0}: L^{2}\left(\mathbf{R}^{2}\right) \rightarrow \mathcal{E}_{0}, \quad P_{0}^{2}=P_{0}, \quad P_{0}^{*}=P_{0}
$$

be the orthogonal projection onto the zero eigenstate $\mathcal{E}_{0}$ of $H$.

Lemma 6.5. Let $G_{2}$ be as in Lemma 5.7. Then $P_{0} W G_{2} W P_{0}=P_{0}$.

Proof. Since $P_{0} f=-G_{0} W P_{0} f \in L^{2}\left(\mathbf{R}^{2}\right)$ for $f \in L^{2}\left(\mathbf{R}^{2}\right)$, we obtain by Lemma 5.9 that $\left(W P_{0} f, \tilde{u}_{l}\right)=0$ for $l=0,1$. Hence it follows from Lemma 5.8 that

$$
\left(P_{0} W G_{2} W P_{0} f, g\right)=\left(G_{2} W P_{0} f, W P_{0} g\right)=\left(P_{0} f, g\right)
$$

for any $f, g \in L^{2}\left(\mathbf{R}^{2}\right)$. This proves the lemma. 
Lemma 6.6. Let $D_{0}: L^{2}\left(\mathbf{R}^{2}\right) \rightarrow L^{2}\left(\mathbf{R}^{2}\right)$ be defined by $D_{0}=$ $\chi_{L} P_{0} W G_{2} W Q$. Then $D_{0}$ has the following properties:

$$
D_{0}^{2}=D_{0}, \quad D_{0} Q=D_{0}, \quad Q D_{0}=D_{0} .
$$

Proof. We first note that $Q \chi_{L} P_{0}=\chi_{L} P_{0}$, which is an immediate consequence of Lemma 6.3. Hence a simple calculation using Lemma 6.5 yields the relations in the lemma.

The above lemma enables us to define the family of projections as

$$
\Gamma_{1}=\left(\mathrm{Id}-D_{0}\right) Q, \quad \Gamma_{2}=D_{0} Q, \quad \Gamma_{3}=\mathrm{Id}-Q
$$

with the properties

$$
\Gamma_{1}+\Gamma_{2}+\Gamma_{3}=\mathrm{Id}, \quad \Gamma_{i} \Gamma_{j}=\delta_{i j} \Gamma_{j}
$$

$\delta_{i j}$ being the Kronecker notation. We see that $\Gamma_{2}: L^{2}\left(\mathbf{R}^{2}\right) \rightarrow \mathcal{M}_{0}$ is the projection on $\mathcal{M}_{0}$. This follows from Lemma 6.3, because $\chi_{L} P_{0}=D_{0} \chi_{L} P_{0}$ by Lemma 6.5. We decompose $\mathcal{M}$ and $\mathcal{E}$ as $\mathcal{M}=\mathcal{M}_{0} \oplus \mathcal{M}_{1}$ and $\mathcal{E}=\mathcal{E}_{0} \oplus \mathcal{E}_{1}$, where $\mathcal{M}_{1}=\Gamma_{1}(\mathcal{M})$ and $\mathcal{E}_{1}=G_{0} W\left(\mathcal{M}_{1}\right)$. By Lemma 6.3 again, $\operatorname{dim} \mathcal{M}_{1}=$ $\operatorname{dim} \mathcal{E}_{1} \leq 2$, and the mappings $G_{0} W: \mathcal{M}_{1} \rightarrow \mathcal{E}_{1}$ and $\chi_{L}: \mathcal{E}_{1} \rightarrow \mathcal{M}_{1}$ are injective and surjective. An element $u \in \mathcal{E}_{1}, u \notin L^{2}\left(\mathbf{R}^{2}\right)$, is called a resonance state at energy zero of $H$.

LEMMA 6.7.

(1) $\left(\mathrm{Id}+\chi_{L} G_{0} W\right) \Gamma_{1}=0, \quad\left(\operatorname{Id}+\chi_{L} G_{0} W\right) \Gamma_{2}=0$.

(2) $\Gamma_{1}^{*} W\left(\operatorname{Id}+\chi_{L} G_{0} W\right)=0, \quad \Gamma_{2}^{*} W\left(\mathrm{Id}+\chi_{L} G_{0} W\right)=0$.

Proof. (1) is obvious by definition, and (2) easily follows by adjoint, if (6.1) is taken into account.

LEMMA 6.8. Let $G_{1}( \pm k)=Z_{0}( \pm k)+Z_{1}( \pm k)$ be as in Lemma 5.7. Then one has $\Gamma_{2}^{*} W Z_{l}( \pm k) W \Gamma_{2}=0$ and

$$
\begin{array}{ll}
\Gamma_{1}^{*} W Z_{l}( \pm k) W \Gamma_{2}=0, & \Gamma_{2}^{*} W Z_{l}( \pm k) W \Gamma_{1}=0, \\
\Gamma_{3} W Z_{l}( \pm k) W \Gamma_{2}=0, & \Gamma_{2}^{*} W Z_{l}( \pm k) W \Gamma_{3}=0 .
\end{array}
$$

Proof. If $u \in \mathcal{M}_{0}$, then $\left(T_{l} U_{l} W u, u_{l}\right)_{L^{2}\left(\mathbf{R}_{+}\right)}=\left(u, W \tilde{u}_{l}\right)_{L^{2}\left(\mathbf{R}^{2}\right)}=0$. This, together with (5.3), implies that

$$
Z_{l}( \pm k) W u=U_{l}^{*} F_{l 1}( \pm k) U_{l} W u=U_{l}^{*} T_{l}^{*} E_{l 1}( \pm k) T_{l}( \pm k) U_{l} W u=0
$$

and hence $Z_{l}( \pm k) W \Gamma_{2}=0$. Since $Z_{l}^{*}(k)=Z_{l}(-k)$, we have $\Gamma_{2}^{*} W Z_{l}( \pm k)$ $=0$ by adjoint. Thus all the relations in the lemma follow at once. 
LEMMA 6.9.

$$
\Gamma_{2}^{*} W G_{2} W \Gamma_{1}=0, \quad \Gamma_{1}^{*} W G_{2} W \Gamma_{2}=0 .
$$

Proof. The second relation follows from the first one by adjoint. To prove the first relation, we calculate:

$$
\begin{aligned}
\left(W G_{2} W \Gamma_{1} f, \Gamma_{2} g\right) & =\left(W G_{2} W \Gamma_{1} f, \chi_{L} P_{0} W G_{2} W Q g\right) \\
& =\left(\chi_{L} P_{0} W G_{2} W \Gamma_{1} f, W G_{2} W Q g\right) \\
& =\left(D_{0} Q \Gamma_{1} f, W G_{2} W Q g\right) \\
& =\left(\Gamma_{2} \Gamma_{1} f, W G_{2} W Q g\right)=0
\end{aligned}
$$

for any $f, g \in L^{2}\left(\mathbf{R}^{2}\right)$. This completes the proof.

LEMmA 6.10. (1) $\Gamma_{3}\left(\mathrm{Id}+\chi_{L} G_{0} W\right) \Gamma_{3}$ has the bounded inverse

$$
Y_{3}=\left(\Gamma_{3}\left(\mathrm{Id}+\chi_{L} G_{0} W\right) \Gamma_{3}\right)^{-1}: \operatorname{Ran} \Gamma_{3} \longrightarrow \operatorname{Ran} \Gamma_{3} .
$$

(2) $\Gamma_{2}^{*} W G_{2} W \Gamma_{2}$ has the bounded inverse

$$
Y_{2}=\left(\Gamma_{2}^{*} W G_{2} W \Gamma_{2}\right)^{-1}: \operatorname{Ran} \Gamma_{2}^{*} \longrightarrow \operatorname{Ran} \Gamma_{2} .
$$

Proof. (1) This is nothing but (6.3). (2) Since $\operatorname{dim} \operatorname{Ran} \Gamma_{2}=\operatorname{dim} \operatorname{Ran} \Gamma_{2}^{*}$ $<\infty$, it is enough to show that: If $\Gamma_{2}^{*} W G_{2} W \Gamma_{2} u=0$ for $u \in L^{2}\left(\mathbf{R}^{2}\right)$, then $\Gamma_{2} u=0$. Since $\Gamma_{2} u \in \mathcal{M}_{0},\left(W \Gamma_{2} u, \tilde{u}_{l}\right)=0$ for $l=0,1$. Hence it follows from Lemma 5.8 that

$$
\left(G_{0} W \Gamma_{2} u, G_{0} W \Gamma_{2} u\right)=\left(G_{2} W \Gamma_{2} u, W \Gamma_{2} u\right)=0
$$

so that $G_{0} W \Gamma_{2} u=0$. By Lemma $6.3, G_{0} W: \mathcal{M}_{0} \rightarrow \mathcal{E}_{0}$ is injective. This yields $\Gamma_{2} u=0$ and the proof is complete.

6.2. We analyse the inversion of $\Gamma_{1}^{*} W G_{1}(k) W \Gamma_{1}: \operatorname{Ran} \Gamma_{1} \rightarrow \operatorname{Ran} \Gamma_{1}^{*}$. This is separately done according as $\operatorname{dim} \mathcal{E}_{1}=0,1$ or 2 . We first discuss the case $\operatorname{dim} \mathcal{E}_{1}=2$. Let $P_{1}: L^{2}\left(\mathbf{R}^{2}\right) \rightarrow \mathcal{M}_{1}$ be the orthogonal projection on $\mathcal{M}_{1}, \operatorname{dim} \mathcal{M}_{1}=2$. We set

$$
\psi_{l}=P_{1} W \tilde{u}_{l} \in \mathcal{M}_{1}, \quad l=0,1
$$

for $\tilde{u}_{l}$ defined by (5.10). Then $\psi_{0}$ and $\psi_{1}$ are linearly independent and span $\mathcal{M}_{1}$. We further introduce $\eta_{l} \in \mathcal{M}_{1}, l=0,1$, with property $\left(\psi_{i}, \eta_{j}\right)=\delta_{i j}$ 
for $0 \leq i, j \leq 1$, and we define the projection $Q_{l}, l=0,1$, as $Q_{l}=\left(\cdot, \eta_{l}^{*}\right) \eta_{l}$, where $\eta_{l}^{*} \in L^{2}\left(\mathbf{R}^{2}\right)$ denotes the basis dual to $\eta_{l}$ such that $\left(\eta_{i}, \eta_{j}^{*}\right)=\delta_{i j}$ and

$$
\eta_{l}^{*} \perp \operatorname{Ran} \Gamma_{2} \oplus \operatorname{Ran} \Gamma_{3}, \quad \operatorname{Ran} \Gamma_{2}=\mathcal{M}_{0} .
$$

The projection $Q_{l}, l=0,1$, is easily shown to have the following properties:

$$
Q_{0}+Q_{1}=\Gamma_{1}, \quad Q_{i} Q_{j}=\delta_{i j} Q_{j}, \quad 0 \leq i, j \leq 1
$$

LEMMA 6.11. If $i \neq j, 0 \leq i, j \leq 1$, then

$$
Z_{i}( \pm k) W Q_{j}=0, \quad Q_{j}^{*} W Z_{i}( \pm k)=0 .
$$

Proof. If $i \neq j$, then

$$
\left(T_{i} U_{i} W \eta_{j}, u_{i}\right)_{L^{2}\left(\mathbf{R}_{+}\right)}=\left(\eta_{j}, W \tilde{u}_{i}\right)_{L^{2}\left(\mathbf{R}^{2}\right)}=\left(\eta_{j}, P_{1} W \tilde{u}_{i}\right)=\left(\eta_{j}, \psi_{i}\right)=0 .
$$

This, together with (5.3), implies that $E_{i 1}( \pm k) T_{i}( \pm k) U_{i} W \eta_{j}=0$, and hence

$$
Z_{i}( \pm k) W Q_{j} f=\left(f, \eta_{j}^{*}\right) U_{i}^{*} T_{i}^{*} E_{i 1}( \pm k) T_{i}( \pm k) U_{i} W \eta_{j}=0
$$

for $f \in L^{2}\left(\mathbf{R}^{2}\right)$. Thus the first relation is obtained. The second one follows by adjoint at once.

LemMA 6.12. $\quad Q_{l}^{*} W Z_{l}(k) W Q_{l}, l=0,1$, takes the form

$$
Q_{l}^{*} W Z_{l}(k) W Q_{l}=\gamma_{l}(k)\left(1+O\left(k^{2 \nu}\right)\right)\left(\cdot, \eta_{l}^{*}\right) \eta_{l}^{*}
$$

and it has the inverse of the form

$$
\left(Q_{l}^{*} W Z_{l}(k) W Q_{l}\right)^{-1}=k^{-2 \nu}\left(1+O\left(k^{2 \nu}\right)\right) Y_{l}: \operatorname{Ran} Q_{l}^{*} \longrightarrow \operatorname{Ran} Q_{l},
$$

where $Y_{l}=\kappa_{l}\left(\cdot, \eta_{l}\right) \eta_{l}$ with

$$
\kappa_{l}=-((l+\nu) \Gamma(1+\nu) / 2 \nu \Gamma(1-\nu)) 2^{2 \nu} e^{i \nu \pi}, \quad l=0,1 .
$$

Proof. By (5.4), we have

$$
\begin{aligned}
\left(T_{l}(k) U_{l} W \eta_{l}, u_{l}\right)_{L^{2}\left(\mathbf{R}_{+}\right)} & =\left(T_{l} U_{l} W \eta_{l}, u_{l}\right)_{L^{2}\left(\mathbf{R}_{+}\right)}+O\left(k^{2 \nu}\right) \\
& =\left(\eta_{l}, \psi_{l}\right)_{L^{2}\left(\mathbf{R}^{2}\right)}+O\left(k^{2 \nu}\right)=1+O\left(k^{2 \nu}\right) .
\end{aligned}
$$

This, together with Lemma 5.7 (3), yields that

$$
\begin{aligned}
Q_{l}^{*} W Z_{l}(k) W Q_{l} f & =\gamma_{l}(k)\left(f, \eta_{l}^{*}\right)\left(T_{l}(k) U_{l} W \eta_{l}, u_{l}\right)_{L^{2}\left(\mathbf{R}_{+}\right)}\left(W \tilde{u}_{l}, \eta_{l}\right) \eta_{l}^{*} \\
& =\gamma_{l}(k)\left(1+O\left(k^{2 \nu}\right)\right)\left|\left(\psi_{l}, \eta_{l}\right)\right|^{2}\left(f, \eta_{l}^{*}\right) \eta_{l}^{*}
\end{aligned}
$$

for any $f \in L^{2}\left(\mathbf{R}^{2}\right)$. Thus $Q_{l}^{*} W Z_{l}(k) W Q_{l}$ takes the desired form. By (4.5), $1 / \gamma_{l}(k)$ behaves like $1 / \gamma_{l}(k)=\kappa_{l} k^{-2 \nu}\left(1+O\left(k^{2 \nu}\right)\right)$ as $k \rightarrow 0$. Hence the inverse also has the form in the lemma and the proof is complete. 
We set $Q_{2}=\Gamma_{2}$ and $Q_{3}=\Gamma_{3}$. Then the family $\left\{Q_{j}\right\}_{j=0}^{3}$ of projections has properties similar to those in (6.5). We are now in a position to apply Proposition 6.1 to the operator

$$
A=\mathrm{Id}+\chi_{L} R\left(k^{2}+i 0 ; K_{\alpha}\right) W: \mathcal{X}_{1} \longrightarrow \mathcal{Y}_{1}
$$

with $\mathcal{X}_{1}=\mathcal{Y}_{1}=L^{2}\left(\mathbf{R}^{2}\right)$ under the situation

$$
\begin{aligned}
& B=\left(k^{-\alpha} Q_{0}, k^{-(1-\alpha)} Q_{1}, k^{-1} Q_{2}, Q_{3}\right): \mathcal{X}_{0} \longrightarrow \mathcal{X}_{1} \\
& C={ }^{t}\left(k^{-\alpha} Q_{0}^{*} W, k^{-(1-\alpha)} Q_{1}^{*} W, k^{-1} Q_{2}^{*} W, Q_{3}\right): \mathcal{Y}_{1} \longrightarrow \mathcal{Y}_{0} \text {, }
\end{aligned}
$$

where

$$
\mathcal{X}_{0}=\sum_{j=0}^{3} \oplus \operatorname{Ran} Q_{j}, \quad \mathcal{Y}_{0}=\left(\sum_{j=0}^{2} \oplus \operatorname{Ran} Q_{j}^{*}\right) \oplus \operatorname{Ran} Q_{3} .
$$

By Lemmas 5.7 and 5.10, $A$ has an asymptotic expansion in $B\left(\mathcal{X}_{1} \rightarrow \mathcal{Y}_{1}\right)$, and it follows from Lemmas $6.7 \sim 6.12$ that the matrix notation $\mathcal{A}=C A B$ : $\mathcal{X}_{0} \rightarrow \mathcal{Y}_{0}$ associated with $A$ has the inverse of the form

$$
\mathcal{A}^{-1}=\left(\begin{array}{cccc}
Y_{0} & 0 & 0 & 0 \\
0 & Y_{1} & 0 & 0 \\
0 & 0 & Y_{2} & 0 \\
0 & 0 & 0 & Y_{3}
\end{array}\right)+O p\left(o\left(k^{0}\right)\right)
$$

in $B\left(\mathcal{Y}_{0} \rightarrow \mathcal{X}_{0}\right)$. It is obvious that $B$ is surjective. We shall show that $C$ is injective. Assume that $C u=0$ for $u \in L^{2}\left(\mathbf{R}^{2}\right)$. Since $Q_{3} u=0, u$ is in $\mathcal{M}$ and hence $Q u=u$. We further have $Q^{*} W u=\left(Q_{0}^{*}+Q_{1}^{*}+Q_{2}^{*}\right) W u=0$. This implies that

$$
\left(W u, G_{0} W u\right)=\left(W u, \chi_{L} G_{0} W u\right)=-(W u, u)=-(W u, Q u)=0 .
$$

If we set $v=G_{0} W u$, then $v \in L_{-1}^{2}\left(\mathbf{R}^{2}\right)$ and $\left(K_{\alpha} v, v\right)=0$. We can obtain $v=0$ by the same argument as in the proof of Proposition 5.1, and hence it follows that $u=-\chi_{L} G_{0} W u=-\chi_{L} v=0$. Thus $C$ is shown to be injective. Hence the inverse $A^{-1}=B \mathcal{A}^{-1} C$ is expanded as

$$
\begin{aligned}
A^{-1}= & k^{-2 \alpha} Q_{0} Y_{0} Q_{0}^{*} W+k^{-2(1-\alpha)} Q_{1} Y_{1} Q_{1}^{*} W \\
& +k^{-2} Q_{2} Y_{2} Q_{2}^{*} W+Q_{3} Y_{3} Q_{3}+B\left(O p\left(o\left(k^{0}\right)\right)\right) C,
\end{aligned}
$$


so that $R\left(k^{2}+i 0 ; H\right)$ takes the form

$$
\begin{aligned}
& \chi_{L} R\left(k^{2}+i 0 ; H\right) \chi_{L} \\
& =\left(k^{-2 \alpha} Q_{0} Y_{0} Q_{0}^{*}+k^{-2(1-\alpha)} Q_{1} Y_{1} Q_{1}^{*}\right) W R\left(k^{2}+i 0 ; K_{\alpha}\right) \chi_{L} \\
& \quad+\left(k^{-2} Q_{2} Y_{2} Q_{2}^{*} W+Q_{3} Y_{3} Q_{3}\right) \chi_{L} R\left(k^{2}+i 0 ; K_{\alpha}\right) \chi_{L} \\
& \quad+B\left(O p\left(o\left(k^{0}\right)\right)\right) C \chi_{L} R\left(k^{2}+i 0 ; K_{\alpha}\right) \chi_{L} .
\end{aligned}
$$

6.3. Next we consider the case $\operatorname{dim} \mathcal{E}_{1}=1$. Let $P_{1}: L^{2}\left(\mathbf{R}^{2}\right) \rightarrow \mathcal{M}_{1}$ be again the orthogonal projection on $\mathcal{M}_{1}$ and let $\psi_{l}, l=0,1$, be defined by (6.6). Since $\operatorname{dim} \mathcal{M}_{1}=1$, at least one of $\psi_{0}$ and $\psi_{1}$ never vanishes. Assume that $\psi_{0} \neq 0$. Then $\psi_{0}$ spans $\mathcal{M}_{1}$, and there exists $\sigma \in \mathbf{C}$ such that

$$
\psi_{1}=\sigma \psi_{0}
$$

We normalize $\psi_{0}$ as $\eta_{0}=\psi_{0} /\left(\psi_{0}, \psi_{0}\right) \in \mathcal{M}_{1}$, so that $\left(\psi_{0}, \eta_{0}\right)=1$ and hence $\left(\psi_{1}, \eta_{0}\right)=\sigma$. We further denote by $\eta_{0}^{*} \in L^{2}\left(\mathbf{R}^{2}\right)$ the basis dual to $\eta_{0}$ such that $\left(\eta_{0}, \eta_{0}^{*}\right)=1$ and $\eta_{0}^{*} \perp \operatorname{Ran} \Gamma_{2} \oplus \operatorname{Ran} \Gamma_{3}$, and we define the projection $\tilde{Q}_{0}=\left(\cdot, \eta_{0}^{*}\right) \eta_{0}: L^{2}\left(\mathbf{R}^{2}\right) \rightarrow \mathcal{M}_{1}$. If $\psi_{0}=0$ (and hence $\left.\psi_{1} \neq 0\right)$, then we take $\eta_{1}$ and $\eta_{1}^{*}$ in the same way as above and define $\tilde{Q}_{1}=\left(\cdot, \eta_{1}^{*}\right) \eta_{1}: L^{2}\left(\mathbf{R}^{2}\right) \rightarrow \mathcal{M}_{1}$.

Lemma 6.13. (1) Assume that $\psi_{0}=P_{1} W \tilde{u}_{0} \neq 0$. Let $\sigma$ be as above. Then $\tilde{Q}_{0}^{*} W G_{1}(k) W \tilde{Q}_{0}$ has the inverse of the form

$$
\left(\tilde{Q}_{0}^{*} W G_{1}(k) W \tilde{Q}_{0}\right)^{-1}=(1+o(1)) Y_{0}(k): \operatorname{Ran} \tilde{Q}_{0}^{*} \longrightarrow \operatorname{Ran} \tilde{Q}_{0},
$$

where $Y_{0}(k)=\left(\gamma_{0}(k)+|\sigma|^{2} \gamma_{1}(k)\right)^{-1}\left(\cdot, \eta_{0}\right) \eta_{0}$.

(2) If $\psi_{0}=0$, then

$$
\left(\tilde{Q}_{1}^{*} W G_{1}(k) W \tilde{Q}_{1}\right)^{-1}=(1+o(1)) Y_{1}(k): \operatorname{Ran} \tilde{Q}_{1}^{*} \longrightarrow \operatorname{Ran} \tilde{Q}_{1},
$$

where $Y_{1}(k)=\gamma_{1}(k)^{-1}\left(\cdot, \eta_{1}\right) \eta_{1}$.

Proof. We prove (1) only. (2) is verified in the same way. As in the proof of Lemma 6.12, we calculate

$$
\begin{aligned}
& \tilde{Q}_{0}^{*} W Z_{0}(k) W \tilde{Q}_{0} f=\gamma_{0}(k)(1+o(1))\left|\left(\psi_{0}, \eta_{0}\right)\right|^{2}\left(f, \eta_{0}^{*}\right) \eta_{0}^{*} \\
& \tilde{Q}_{0}^{*} W Z_{1}(k) W \tilde{Q}_{0} f=\gamma_{1}(k)(1+o(1))\left|\left(\psi_{1}, \eta_{0}\right)\right|^{2}\left(f, \eta_{0}^{*}\right) \eta_{0}^{*}
\end{aligned}
$$

for $f \in L^{2}\left(\mathbf{R}^{2}\right)$. Thus the desired relation is obtained. 
We discuss the case $\alpha=1 / 2$ in detail. Assume that $\psi_{0} \neq 0$. If $\alpha=1 / 2$, then $\nu=|l-\alpha|=1 / 2$ for $l=0,1$, and the operator $Y_{0}(k)$ in Lemma 6.13 is represented as

$$
Y_{0}(k)=k^{-1}(1+o(1)) \tilde{Y}_{0},
$$

where

$$
\tilde{Y}_{0}=\kappa\left(\cdot, \eta_{0}\right) \eta_{0}, \quad \kappa=-(\Gamma(3 / 2) / \Gamma(1 / 2))\left(1+|\sigma|^{2} / 3\right)^{-1} e^{i \pi / 2} .
$$

We again set $Q_{2}=\Gamma_{2}$ and $Q_{3}=\Gamma_{3}$, and apply Proposition 6.1 to

$$
A=\mathrm{Id}+\chi_{L} R\left(k^{2}+i 0 ; K_{\alpha}\right) W: \mathcal{X}_{1} \longrightarrow \mathcal{Y}_{1}
$$

with $\mathcal{X}_{1}=\mathcal{Y}_{1}=L^{2}\left(\mathbf{R}^{2}\right)$ under the situation

$$
\begin{aligned}
& B=\left(k^{-1 / 2} \tilde{Q}_{0}, k^{-1} Q_{2}, Q_{3}\right): \mathcal{X}_{0} \longrightarrow \mathcal{X}_{1}, \\
& C={ }^{t}\left(k^{-1 / 2} \tilde{Q}_{0}^{*} W, k^{-1} Q_{2}^{*} W, Q_{3}\right): \mathcal{Y}_{1} \longrightarrow \mathcal{Y}_{0}
\end{aligned}
$$

where

$$
\mathcal{X}_{0}=\operatorname{Ran} \tilde{Q}_{0} \oplus \operatorname{Ran} Q_{2} \oplus \operatorname{Ran} Q_{3}, \quad \mathcal{Y}_{0}=\operatorname{Ran} \tilde{Q}_{0}^{*} \oplus \operatorname{Ran} Q_{2}^{*} \oplus \operatorname{Ran} Q_{3} .
$$

Then the matrix notation $\mathcal{A}=C A B$ has the inverse of the form

$$
\mathcal{A}^{-1}=\left(\begin{array}{ccc}
\tilde{Y}_{0} & 0 & 0 \\
0 & Y_{2} & 0 \\
0 & 0 & Y_{3}
\end{array}\right)+O p\left(o\left(k^{0}\right)\right)
$$

in $B\left(\mathcal{Y}_{0} \rightarrow \mathcal{X}_{0}\right)$, and $A^{-1}$ is expanded as

$$
A^{-1}=k^{-1} \tilde{Q}_{0} \tilde{Y}_{0} \tilde{Q}_{0}^{*} W+k^{-2} Q_{2} Y_{2} Q_{2}^{*} W+Q_{3} Y_{3} Q_{3}+B\left(O p\left(o\left(k^{0}\right)\right)\right) C .
$$

Thus we have

$$
\begin{aligned}
\chi_{L} R\left(k^{2}+i 0 ;\right. & H) \chi_{L}=k^{-1} \tilde{Q}_{0} \tilde{Y}_{0} \tilde{Q}_{0}^{*} W R\left(k^{2}+i 0 ; K_{\alpha}\right) \chi_{L} \\
& +\left(k^{-2} Q_{2} Y_{2} Q_{2}^{*} W+Q_{3} Y_{3} Q_{3}\right) \chi_{L} R\left(k^{2}+i 0 ; K_{\alpha}\right) \chi_{L} \\
& +B\left(O p\left(o\left(k^{0}\right)\right)\right) C \chi_{L} R\left(k^{2}+i 0 ; K_{\alpha}\right) \chi_{L}
\end{aligned}
$$

provided that $\alpha=1 / 2$ and $\psi_{0} \neq 0$. If $\psi_{0}=0$, the operator $Y_{1}(k)$ in Lemma 6.13 takes the form

$$
Y_{1}(k)=k^{-1}(1+o(1)) \tilde{Y}_{1}, \quad \tilde{Y}_{1}=\tilde{\kappa}\left(\cdot, \eta_{1}\right) \eta_{1}
$$


with $\tilde{\kappa}=-(3 \Gamma(3 / 2) / \Gamma(1 / 2)) e^{i \pi / 2}$. Similarly we obtain

$$
\begin{aligned}
\chi_{L} R\left(k^{2}+i 0 ; H\right) \chi_{L}= & k^{-1} \tilde{Q}_{1} \tilde{Y}_{1} \tilde{Q}_{1}^{*} W R\left(k^{2}+i 0 ; K_{\alpha}\right) \chi_{L} \\
& +\left(k^{-2} Q_{2} Y_{2} Q_{2}^{*} W+Q_{3} Y_{3} Q_{3}\right) \chi_{L} R\left(k^{2}+i 0 ; K_{\alpha}\right) \chi_{L} \\
& +B\left(O p\left(o\left(k^{0}\right)\right)\right) C \chi_{L} R\left(k^{2}+i 0 ; K_{\alpha}\right) \chi_{L} .
\end{aligned}
$$

We briefly discuss the case $\alpha \neq 1 / 2$. Let $0<\alpha<1 / 2$ and assume that $\psi_{0} \neq 0$. Then $\alpha<1-\alpha$ and $Y_{0}(k)$ takes the form

$$
Y_{0}(k)=k^{-2 \alpha}(1+o(1)) \tilde{Y}_{0 \alpha}
$$

where

$$
\tilde{Y}_{0 \alpha}=\kappa_{\alpha}\left(\cdot, \eta_{0}\right) \eta_{0}, \quad \kappa_{\alpha}=-(\Gamma(1+\alpha) / 2 \Gamma(1-\alpha)) 2^{2 \alpha} e^{i \alpha \pi}
$$

We repeat the same argument as in the case $\alpha=1 / 2$ to obtain that

$$
\begin{aligned}
\chi_{L} R\left(k^{2}+i 0 ; H\right) \chi_{L}= & k^{-2 \alpha} \tilde{Q}_{0} \tilde{Y}_{0 \alpha} \tilde{Q}_{0}^{*} W R\left(k^{2}+i 0 ; K_{\alpha}\right) \chi_{L} \\
& +\left(k^{-2} Q_{2} Y_{2} Q_{2}^{*} W+Q_{3} Y_{3} Q_{3}\right) \chi_{L} R\left(k^{2}+i 0 ; K_{\alpha}\right) \chi_{L} \\
& +B\left(O p\left(o\left(k^{0}\right)\right)\right) C \chi_{L} R\left(k^{2}+i 0 ; K_{\alpha}\right) \chi_{L},
\end{aligned}
$$

where

$$
B=\left(k^{-\alpha} \tilde{Q}_{0}, k^{-1} Q_{2}, Q_{3}\right), \quad C={ }^{t}\left(k^{-\alpha} \tilde{Q}_{0}^{*} W, k^{-1} Q_{2}^{*} W, Q_{3}\right)
$$

If $\psi_{0}=0$, then

$$
Y_{1}(k)=k^{-2(1-\alpha)}(1+o(1)) \tilde{Y}_{1 \alpha}, \quad \tilde{Y}_{1 \alpha}=\tilde{\kappa}_{\alpha}\left(\cdot, \eta_{1}\right) \eta_{1}
$$

with

$$
\tilde{\kappa}_{\alpha}=-((2-\alpha) \Gamma(2-\alpha) / 2(1-\alpha) \Gamma(\alpha)) 2^{2(1-\alpha)} e^{i(1-\alpha) \pi}
$$

and we have

$$
\begin{aligned}
\chi_{L} R\left(k^{2}+i 0 ;\right. & H) \chi_{L}=k^{-2(1-\alpha)} \tilde{Q}_{1} \tilde{Y}_{1 \alpha} \tilde{Q}_{1}^{*} W R\left(k^{2}+i 0 ; K_{\alpha}\right) \chi_{L} \\
& +\left(k^{-2} Q_{2} Y_{2} Q_{2}^{*} W+Q_{3} Y_{3} Q_{3}\right) \chi_{L} R\left(k^{2}+i 0 ; K_{\alpha}\right) \chi_{L} \\
& +B\left(O p\left(o\left(k^{0}\right)\right)\right) C \chi_{L} R\left(k^{2}+i 0 ; K_{\alpha}\right) \chi_{L}
\end{aligned}
$$

where

$$
B=\left(k^{-(1-\alpha)} \tilde{Q}_{1}, k^{-1} Q_{2}, Q_{3}\right), \quad C={ }^{t}\left(k^{-(1-\alpha)} \tilde{Q}_{1}^{*} W, k^{-1} Q_{2}^{*} W, Q_{3}\right) .
$$


A similar argument applies to the case $1 / 2<\alpha<1$. We skip the details.

Finally we consider the case $\operatorname{dim} \mathcal{E}_{1}=0$. This is the most simple case. If we again repeat the same argument as above, we have

$$
\begin{aligned}
& \chi_{L} R\left(k^{2}+i 0 ; H\right) \chi_{L} \\
& =\left(k^{-2} Q_{2} Y_{2} Q_{2}^{*} W+Q_{3} Y_{3} Q_{3}\right) \chi_{L} R\left(k^{2}+i 0 ; K_{\alpha}\right) \chi_{L} \\
& \quad+B\left(O p\left(o\left(k^{0}\right)\right)\right) C \chi_{L} R\left(k^{2}+i 0 ; K_{\alpha}\right) \chi_{L},
\end{aligned}
$$

where $B=\left(k^{-1} Q_{2}, Q_{3}\right)$ and $C={ }^{t}\left(k^{-1} Q_{2}^{*} W, Q_{3}\right)$.

\section{$\S 7$. Behavior at low energy of scattering amplitude}

In this section we study the behavior as $\lambda \rightarrow 0$ of the scattering amplitude $f\left(\omega \rightarrow \omega^{\prime} ; \lambda\right)$. The argument is again divided into the three cases according as $\operatorname{dim} \mathcal{E}_{1}=0,1$ or 2 . Let $D_{M}=\left[H_{\alpha}, \chi_{\infty M}\right]$ be defined by (3.6), $M>2$ being fixed. We again take $L \gg 1$ so large that $\chi_{L} D_{M}=D_{M} \chi_{L}=$ $D_{M}$.

7.1. We first discuss the case $\operatorname{dim} \mathcal{E}_{1}=2$. We set ourselves under the situation in subsection 6.2 and use the same notations with the meanings ascribed there. We define

$$
\rho_{l}=G_{0} W \eta_{l}, \quad l=0,1 .
$$

Since $\eta_{l} \in \mathcal{M}_{1}$, it follows from Lemmas 6.2 and 6.3 that $\rho_{l} \in \mathcal{E}_{1}$, and Lemma 5.9 implies that $\rho_{l}$ takes the form

$$
\rho_{l}=\sum_{j \in \mathbf{Z}} U_{j}^{*} F_{j 0} U_{j} W \eta_{l}=\sum_{j=0,1} U_{j}^{*} E_{j 0} T_{j} U_{j} W \eta_{l}+g
$$

for some $g \in L^{2}\left(\mathbf{R}^{2}\right)$. By (5.6), we have

$$
\left(T_{j} U_{j} W \eta_{l}\right)(r)=\left(\left(\mathrm{Id}-W_{j} F_{j 0}\right) U_{j} W \eta_{l}\right)(r) .
$$

Recall that $W_{l}(r)=0$ for $r>2$ and the coefficients of the differential operator $W=H-K_{\alpha}$ also vanishes for $r=|x|>2$. Hence we have $\left(T_{j} U_{j} W \eta_{l}\right)(r)=0$ for $r>2$. Let $\left(u_{l}, v_{l}\right)$ be the pair of linearly independent solutions defined in subsection 4.1. If $r>2$, then we calculate

$$
\begin{aligned}
\left(E_{j 0} T_{j} U_{j} W \eta_{l}\right)(r) & =\left(T_{j} U_{j} W \eta_{l}, u_{j}\right)_{L^{2}\left(\mathbf{R}_{+}\right)} v_{j}(r) \\
& =\left(\eta_{l}, W U_{j}^{*} T_{j}^{*} u_{j}\right)_{L^{2}\left(\mathbf{R}^{2}\right)} v_{j}(r) \\
& =\left(\eta_{l}, W \tilde{u}_{j}\right) v_{j}(r)=\left(\eta_{l}, \psi_{j}\right) v_{j}(r)=\delta_{l j} v_{j}(r)
\end{aligned}
$$


for $j=0,1$. Thus $\rho_{l}(x)$ is represented as

$$
\begin{aligned}
\rho_{l}(x) & =\left(U_{l}^{*} v_{l}\right)(x)+g=(2 \pi)^{-1 / 2} r^{-1 / 2} v_{l}(r) e^{i l \theta}+g \\
& =(2 \pi(l+\nu))^{-1 / 2} r^{-\nu} e^{i l \theta}+g
\end{aligned}
$$

with another $g \in L^{2}\left(\mathbf{R}^{2}\right)$. Since $\rho_{l} \in \mathcal{E}_{1}$ satisfies $H \rho_{l}=0$ as a resonance function at energy zero and since

$$
H r^{-\nu} e^{i l \theta}=K_{\alpha} r^{-\nu} e^{i l \theta}=(2 \pi)^{1 / 2} U_{l}^{*} K_{l \alpha} r^{-\nu+1 / 2}=0
$$

for $r=|x|>2$, the remainder term $g$ in (7.1) also satisfies $H g=K_{\alpha} g=0$ for $|x|>2$.

LEMMA 7.1. Let $\varphi_{\mp}(x ; \lambda, \omega)$ be the generalized eigenfunction of $H_{\alpha}$. Then

$$
\begin{aligned}
& \left(\rho_{l}, D_{M} \varphi_{+}(\cdot ; \lambda, \omega)\right)=-(-1)^{l} 2 \nu \mu_{l}(2 \pi /(l+\nu))^{1 / 2} e^{i \nu \pi / 2} e^{i l \omega} k^{\nu}+O(k) \\
& \left(\rho_{l}, D_{M} \varphi_{-}(\cdot ; \lambda, \omega)\right)=-2 \nu \mu_{l}(2 \pi /(l+\nu))^{1 / 2} e^{-i \nu \pi / 2} e^{i l \omega} k^{\nu}+O(k)
\end{aligned}
$$

as $k=\lambda^{1 / 2} \rightarrow 0$, where $\mu_{l}=2^{-\nu} / \Gamma(1+\nu), \nu=|l-\alpha|$.

Proof. We prove the first relation only. The second one can be verified in exactly the same way. For notational brevity, we write $\varphi_{+}$for $\varphi_{+}(x ; \lambda, \omega)$. Since $H_{\alpha}=\left(-i \nabla-B_{\alpha}\right)^{2}$ is rotationally invariant, $D_{M}=\left[H_{\alpha}, \chi_{\infty M}\right]$ is a differential operator of variable $r$ only. By definition (3.4) of $\varphi_{+}$, we have

$$
D_{M} \varphi_{+}=\sum_{l=0,1}(-1)^{l} e^{-i \nu \pi / 2} \mu_{l} e^{i l(\theta-\omega)} k^{\nu} D_{M} r^{\nu}+o_{L^{2}}(k),
$$

where $O_{L^{2}}\left(k^{\gamma}\right)$ denotes a $L^{2}$-valued function of which the $L^{2}$ norm obeys the bound $O\left(k^{\gamma}\right)$ as $k \rightarrow 0$. Since $K_{\alpha}=H_{\alpha}$ for $|x|>M>2$ and $\chi_{\infty M} H_{\alpha} r^{\nu} e^{i l \theta}=0$, it follows that

$$
D_{M} r^{\nu}=e^{-i l \theta} D_{M} e^{i l \theta} r^{\nu}=e^{-i l \theta}\left[H_{\alpha}, \chi_{\infty M}\right] e^{i l \theta} r^{\nu}=e^{-i l \theta} K_{\alpha} e^{i l \theta} \chi_{\infty M} r^{\nu} .
$$

If $g \in L^{2}\left(\mathbf{R}^{2}\right)$ satisfies $H g=K_{\alpha} g=0$ for $|x|>M$, then

$$
\left(g, K_{\alpha} \chi_{\infty M} r^{\nu} e^{i l \theta}\right)=0, \quad l=0,1,
$$

and hence $\left(g, D_{M} \varphi_{+}\right)=o(k)$. Thus the scalar product $\left(\rho_{l}, D_{M} \varphi_{+}\right)$under consideration obeys

$$
\begin{aligned}
& \left(\rho_{l}, D_{M} \varphi_{+}\right)=(-1)^{l}(2 \pi(l+\nu))^{-1 / 2} \mu_{l} e^{i \nu \pi / 2} e^{i l \omega}\left(r^{-\nu} e^{i l \theta}, K_{\alpha} \chi_{\infty} M r^{\nu} e^{i l \theta}\right) k^{\nu} \\
& +o(k)
\end{aligned}
$$


by (7.1). If we take account of relation $K_{l \alpha} r^{-\nu+1 / 2}=0$ for $r>2$, then a simple calculation using integration by parts shows that

$$
\begin{aligned}
\left(r^{-\nu} e^{i l \theta}, K_{\alpha} \chi_{\infty} r^{\nu} e^{i l \theta}\right)_{L^{2}\left(\mathbf{R}^{2}\right)} & =2 \pi\left(r^{-\nu+1 / 2}, K_{l \alpha} \chi_{\infty M} r^{\nu+1 / 2}\right)_{L^{2}\left(\mathbf{R}_{+}\right)} \\
& =-4 \nu \pi .
\end{aligned}
$$

This proves the desired relation.

Lemma 7.2. Let \|\| denote the $L^{2}$ norm in $L^{2}\left(\mathbf{R}^{2}\right)$. Then one has:

(1) $\left\|k^{-\nu} Q_{l}^{*} W R\left(k^{2}+i 0 ; K_{\alpha}\right) D_{M} \varphi_{+}(\cdot ; \lambda, \omega)\right\|=O(1), \quad l=0,1$.

(2) $\left\|k^{-1} Q_{2}^{*} W R\left(k^{2}+i 0 ; K_{\alpha}\right) D_{M} \varphi_{+}(\cdot ; \lambda, \omega)\right\|=o(1)$.

(3) $\left\|Q_{3} \chi_{L} R\left(k^{2}+i 0 ; K_{\alpha}\right) D_{M} \varphi_{+}(\cdot ; \lambda, \omega)\right\|=o(1)$.

Proof. We again write $\varphi_{+}$for $\varphi_{+}(x ; \lambda, \omega)$. (1) We prove this for $l=0$ only. Since $Q_{0}^{*} W Z_{1}(k)=0$ by Lemma 6.11 , we have by Lemmas 5.7 and 5.10 that

$$
\left\|k^{-\alpha} Q_{0}^{*} W R\left(k^{2}+i 0 ; K_{\alpha}\right) D_{M} \varphi_{+}\right\|=\left\|k^{-\alpha} Q_{0}^{*} W G_{0} D_{M} \varphi_{+}\right\|+o(1) .
$$

We calculate

$$
Q_{0}^{*} W G_{0} D_{M} \varphi_{+}=\left(W G_{0} D_{M} \varphi_{+}, \eta_{0}\right) \eta_{0}^{*}=\left(D_{M} \varphi_{+}, \rho_{0}\right) \eta_{0}^{*} .
$$

This, together with Lemma 7.1, implies (1). (2) As is seen from the proof of Lemma 6.8, $Q_{2}^{*} W Z_{l}(k)=0$. Hence it follows again from Lemmas 5.7 and 5.10 that

$$
\left\|k^{-1} Q_{2}^{*} W R\left(k^{2}+i 0 ; K_{\alpha}\right) D_{M} \varphi_{+}\right\|=\left\|k^{-1} Q_{2}^{*} W G_{0} D_{M} \varphi_{+}\right\|+o(1) .
$$

Recall that $Q_{2}\left(=\Gamma_{2}\right): L^{2}\left(\mathbf{R}^{2}\right) \rightarrow \mathcal{M}_{0}$ is the projection on the finite dimensional space $\mathcal{M}_{0}$. If $\psi \in \mathcal{M}_{0}$, then $G_{0} W \psi \in \mathcal{E}_{0} \subset L^{2}\left(\mathbf{R}^{2}\right)$ by Lemma 6.3 and $H G_{0} W \psi=0$. Hence we can show as in the proof of Lemma 7.1 that

$$
\left(W G_{0} D_{M} \varphi_{+}, \psi\right)=\left(D_{M} \varphi_{+}, G_{0} W \psi\right)=o(k) .
$$

This proves (2). (3) This follows from Lemma 5.7 and (7.2) at once.

LEMMA 7.3.

(1) $\left\|k^{-\nu} Q_{l}^{*} D_{M} \varphi_{-}(\cdot ; \lambda, \omega)\right\|=O(1), \quad l=0,1$.

(2) $\left\|k^{-1} Q_{2}^{*} D_{M} \varphi_{-}(\cdot ; \lambda, \omega)\right\|=o(1)$.

(3) $\left\|Q_{3}^{*} D_{M} \varphi_{-}(\cdot ; \lambda, \omega)\right\|=o(1)$. 
Proof. The lemma is verified in almost the same way as Lemma 7.2. (1) Since $\eta_{l} \in \mathcal{M}_{1}$, we have

$$
Q_{l}^{*} D_{M} \varphi_{-}=\left(D_{M} \varphi_{-}, \eta_{l}\right) \eta_{l}^{*}=-\left(D_{M} \varphi_{-}, \chi_{L} G_{0} W \eta_{l}\right) \eta_{l}^{*}=-\left(D_{M} \varphi_{-}, \rho_{l}\right) \eta_{l}^{*}
$$

Hence (1) follows from Lemma 7.1. (2) If $\psi \in \mathcal{M}_{0}$, then $\psi+\chi_{L} G_{0} W \psi=0$ and hence

$$
\left(D_{M} \varphi_{-}, \psi\right)=-\left(D_{M} \varphi_{-}, G_{0} W \psi\right)=o(k) .
$$

This proves (2). (3) This again follows from (7.2) at once.

Let $g_{\alpha}\left(\omega \rightarrow \omega^{\prime} ; \lambda\right)$ be as in Proposition 3.1. We set

$$
I(k)=\left(R(\lambda+i 0 ; H) D_{M} \varphi_{+}(\cdot ; \lambda, \omega), D_{M} \varphi_{-}\left(\cdot ; \lambda, \omega^{\prime}\right)\right) .
$$

Then $\left.g_{\alpha}\left(\omega \rightarrow \omega^{\prime} ; \lambda\right)=G / 4 \pi\right) I(k)$ with $k=\lambda^{1 / 2}$. We analyse the behavior as $k \rightarrow 0$ of the scalar product $I(k)$. Let $B: \mathcal{X}_{0} \rightarrow \mathcal{X}_{1}$ and $C: \mathcal{Y}_{1} \rightarrow \mathcal{Y}_{0}$ be as in (6.7) and (6.8), respectively. If $T(k)$ is of class $o\left(k^{0}\right)$ as a bounded operator in $B\left(\mathcal{Y}_{0} \rightarrow \mathcal{X}_{0}\right)$, then we combine Lemmas 7.2 and 7.3 to obtain that

$$
\left(B T(k) C \chi_{L} R\left(k^{2}+i 0 ; K_{\alpha}\right) D_{M} \varphi_{+}(\cdot ; \lambda, \omega), D_{M} \varphi_{-}\left(\cdot ; \lambda, \omega^{\prime}\right)\right)=o(1)
$$

and also it follows that

$$
\begin{aligned}
k^{-2}\left(Q_{2} Y_{2} Q_{2}^{*} W R\left(k^{2}+i 0 ; K_{\alpha}\right) D_{M} \varphi_{+}(\cdot ; \lambda, \omega), D_{M} \varphi_{-}\left(\cdot ; \lambda, \omega^{\prime}\right)\right) & =o(1) \\
\left(Q_{3} Y_{3} Q_{3} \chi_{L} R\left(k^{2}+i 0 ; K_{\alpha}\right) D_{M} \varphi_{+}(\cdot ; \lambda, \omega), D_{M} \varphi_{-}\left(\cdot ; \lambda, \omega^{\prime}\right)\right) & =o(1)
\end{aligned}
$$

Thus we use the expansion $(6.9)$ of $R\left(k^{2}+i 0 ; H\right)$ to obtain that

$$
\begin{aligned}
& I(k)=\sum_{l=0,1} k^{-2 \nu}\left(Q_{l} Y_{l} Q_{l}^{*} W R\left(k^{2}+i 0 ; K_{\alpha}\right) D_{M} \varphi_{+}(\cdot ; \lambda, \omega), D_{M} \varphi_{-}\left(\cdot ; \lambda, \omega^{\prime}\right)\right) \\
& \quad+o(1)
\end{aligned}
$$

We denote by $I_{l}(k), l=0,1$, the two leading terms on the right side. By Lemmas 5.7, 5.10 and $6.12, I_{l}(k)$ is calculated as follows:

$$
\begin{aligned}
I_{l}(k) & =\kappa_{l} k^{-2 \nu}\left(D_{M} \varphi_{+}(\cdot ; \lambda, \omega), \rho_{l}\right) \times\left(\eta_{l}, D_{M} \varphi_{-}\left(\cdot ; \lambda, \omega^{\prime}\right)\right)+o(1) \\
& =\kappa_{l} k^{-2 \nu}\left(D_{M} \varphi_{+}(\cdot ; \lambda, \omega), \rho_{l}\right) \times\left(-G_{0} W \eta_{l}, D_{M} \varphi_{-}\left(\cdot ; \lambda, \omega^{\prime}\right)\right)+o(1) \\
& =-\kappa_{l} k^{-2 \nu}\left(D_{M} \varphi_{+}(\cdot ; \lambda, \omega), \rho_{l}\right) \times\left(\rho_{l}, D_{M} \varphi_{-}\left(\cdot ; \lambda, \omega^{\prime}\right)\right)+o(1),
\end{aligned}
$$


where $\kappa_{l}$ is the constant in Lemma 6.12. We further make use of Lemma 7.1 to obtain that

$$
I_{l}(k)=\left(-(-1)^{l} \kappa_{l}\left(2 \nu \mu_{l}\right)^{2}(2 \pi /(l+\nu)) e^{-i \nu \pi}+o(1)\right) e^{i l\left(\omega^{\prime}-\omega\right)}
$$

with $\mu_{l}$ as in Lemma 7.1. The leading constant on the right side equals

$$
\begin{aligned}
-(-1)^{l} \kappa_{l}\left(2 \nu \mu_{l}\right)^{2}(2 \pi /(l+\nu)) e^{-i \nu \pi} & =4(-1)^{l} \pi / \Gamma(\nu) \Gamma(1-\nu) \\
& =4(-1)^{l} \sin \nu \pi .
\end{aligned}
$$

Hence Proposition 3.1 shows that

$$
f\left(\omega \rightarrow \omega^{\prime} ; \lambda\right)=c(\lambda)\left(f_{\alpha}\left(\omega^{\prime}-\omega\right)+(i / \pi) \sin \alpha \pi\left(1-e^{i\left(\omega^{\prime}-\omega\right)}\right)+o(1)\right)
$$

with $c(\lambda)=(2 \pi / i \sqrt{\lambda})^{1 / 2}$. Thus the asymptotic formula at low energy of $f\left(\omega \rightarrow \omega^{\prime} ; \lambda\right)$ is established, when $\operatorname{dim} \mathcal{E}_{1}=2$.

7.2. We consider the case $\operatorname{dim} \mathcal{E}_{1}=1$. We set ourselves under the situation in subsection 6.3 and recall the notations there. Let $\psi_{l} \in \mathcal{M}_{1}$, $l=0,1$, be defined by (6.6). If $\psi_{0} \neq 0$, then $\psi_{0}$ spans the one dimensional space $\mathcal{M}_{1}$, and $\psi_{0}$ and $\psi_{1}$ are related through the relation $\psi_{1}=\sigma \psi_{0}$ for some $\sigma \in \mathbf{C}($ see $(6.10))$. Set $\eta_{0}=\psi_{0} /\left(\psi_{0}, \psi_{0}\right) \in \mathcal{M}_{1}$, so that $\left(\psi_{0}, \eta_{0}\right)=1$ and hence $\left(\psi_{1}, \eta_{0}\right)=\sigma$. We now define $\rho_{0}=G_{0} W \eta_{0} \in \mathcal{E}_{1}$. Then $\rho_{0}$ spans $\mathcal{E}_{1}$, and it takes the form

$$
\begin{aligned}
\rho_{0}(x) & =\left(U_{0}^{*} v_{0}\right)(x)+\bar{\sigma}\left(U_{1}^{*} v_{1}\right)(x)+g \\
& =(2 \pi \alpha)^{-1 / 2} r^{-\alpha}+\bar{\sigma}(2 \pi(2-\alpha))^{-1 / 2} r^{-(1-\alpha)} e^{i \theta}+g, \quad g \in L^{2}\left(\mathbf{R}^{2}\right),
\end{aligned}
$$

by the same calculation as in subsection 7.1. If $\psi_{0}=0$ (and hence $\psi_{1} \neq 0$ ), then $\mathcal{E}_{1}$ is spanned by $\rho_{1}=G_{0} W \eta_{1}$ behaving like

$$
\rho_{1}(x)=\left(U_{1}^{*} v_{1}\right)(x)+g=(2 \pi(2-\alpha))^{-1 / 2} r^{-(1-\alpha)} e^{i \theta}+g, \quad g \in L^{2}\left(\mathbf{R}^{2}\right) .
$$

In any case, the resonance space $\mathcal{E}_{1}$ is spanned by a function of the form

$$
\rho(x)=c_{0} r^{-\alpha}+c_{1} r^{-(1-\alpha)} e^{i \theta}+g, \quad g \in L^{2}\left(\mathbf{R}^{2}\right),
$$

with some pair $\left(c_{0}, c_{1}\right)$, where $g$ satisfies $H g=K_{\alpha} g=0$ in $|x|>2$.

We calculate the scalar product $I(k)$ defined above. We again discuss the case $\alpha=1 / 2$ in some detail. Assume that $\psi_{0} \neq 0$. If $\alpha=1 / 2$, then

$$
\rho_{0}(x)=\pi^{-1 / 2} r^{-1 / 2}+\pi^{-1 / 2}(\bar{\sigma} / \sqrt{3}) r^{-1 / 2} e^{i \theta}+g
$$


and hence

$$
c=c_{1} / c_{0}=\bar{\sigma} / \sqrt{3}
$$

for $c_{0} \neq 0$. We make use of (6.12) and repeat the same argument as in subsection 7.1 to obtain that

$$
I(k)=-\kappa k^{-1}\left(D_{M} \varphi_{+}(\cdot ; \lambda, \omega), \rho_{0}\right) \times\left(\rho_{0}, D_{M} \varphi_{-}\left(\cdot ; \lambda, \omega^{\prime}\right)\right)+o(1)
$$

with $\kappa$ as in (6.11). The two scalar products on the right side are calculated as

$$
\begin{aligned}
& \left(\rho_{0}, D_{M} \varphi_{+}(\cdot ; \lambda, \omega)\right)=-\mu e^{i \pi / 4}\left(1-(\bar{\sigma} / \sqrt{3}) e^{i \omega}\right) k^{1 / 2}(1+o(1)) \\
& \left(\rho_{0}, D_{M} \varphi_{-}(\cdot ; \lambda, \omega)\right)=-\mu e^{-i \pi / 4}\left(1+(\bar{\sigma} / \sqrt{3}) e^{i \omega}\right) k^{1 / 2}(1+o(1))
\end{aligned}
$$

in the same way as in the proof of Lemma 7.1 , where $\mu=(2 \pi)^{1 / 2} / \Gamma(3 / 2)$. Thus we have

$$
I(k)=\frac{4}{1+|c|^{2}}\left(1-\bar{c} e^{-i \omega}\right)\left(1+c e^{i \omega^{\prime}}\right)+o(1)
$$

by (7.3). Hence Proposition 3.1 implies that

$$
f\left(\omega \rightarrow \omega^{\prime} ; \lambda\right)=c(\lambda)\left(f_{\alpha}\left(\omega^{\prime}-\omega\right)+\frac{i\left(1-\bar{c} e^{-i \omega}\right)\left(1+c e^{i \omega^{\prime}}\right)}{\pi\left(1+|c|^{2}\right)}+o(1)\right)
$$

when $\alpha=1 / 2$ and $\psi_{0} \neq 0$. Even if $\psi_{0}=0$ (and hence $c_{0}=0$ ), this formula remains true with $c=\infty$.

We briefly discuss the case $\alpha \neq 1 / 2$. Assume that $0<\alpha<1 / 2$. If $\psi_{0} \neq 0$, then we have

$$
I(k)=-\kappa_{\alpha} k^{-2 \alpha}\left(D_{M} \varphi_{+}(\cdot ; \lambda, \omega), \rho_{0}\right) \times\left(\rho_{0}, D_{M} \varphi_{-}\left(\cdot ; \lambda, \omega^{\prime}\right)\right)+o(1)
$$

with $\kappa_{\alpha}$ as in (6.13). Since $\alpha<1-\alpha$, we can calculate the two scalar products on the right side as follows:

$$
\begin{aligned}
& \left(\rho_{0}, D_{M} \varphi_{+}(\cdot ; \lambda, \omega)\right)=-\left(\alpha^{1 / 2} \pi^{1 / 2} / \Gamma(1+\alpha)\right) 2^{3 / 2-\alpha} e^{i \alpha \pi / 2} k^{\alpha}+o\left(k^{\alpha}\right), \\
& \left(\rho_{0}, D_{M} \varphi_{-}(\cdot ; \lambda, \omega)\right)=-\left(\alpha^{1 / 2} \pi^{1 / 2} / \Gamma(1+\alpha)\right) 2^{3 / 2-\alpha} e^{-i \alpha \pi / 2} k^{\alpha}+o\left(k^{\alpha}\right) .
\end{aligned}
$$

This yields that $I(k)=4 \sin \alpha \pi+o(1)$ and hence

$$
f\left(\omega \rightarrow \omega^{\prime} ; \lambda\right)=c(\lambda)\left(f_{\alpha}\left(\omega^{\prime}-\omega\right)+(i / \pi) \sin \alpha \pi+o(1)\right)
$$


If $\psi_{0}=0$, then we have by (6.14) that

$$
f\left(\omega \rightarrow \omega^{\prime} ; \lambda\right)=c(\lambda)\left(f_{\alpha}\left(\omega^{\prime}-\omega\right)-(i / \pi) \sin \alpha \pi e^{i\left(\omega^{\prime}-\omega\right)}+o(1)\right) .
$$

A similar argument applies to the case $1 / 2<\alpha<1$. We mention only the result in the next subsection.

Finally, if $\operatorname{dim} \mathcal{E}_{1}=0$, then we obtain

$$
f\left(\omega \rightarrow \omega^{\prime} ; \lambda\right)=c(\lambda)\left(f_{\alpha}\left(\omega^{\prime}-\omega\right)+o(1)\right)
$$

by $(6.15)$. If $V(x)=0$, then it follows from Proposition 5.1 that $\operatorname{dim} \mathcal{E}_{1}=0$ and hence $f\left(\omega \rightarrow \omega^{\prime} ; \lambda\right)$ obeys the asymptotic formula above.

7.3. We sum up the results obtained above in the main theorem. Assume that $b, V \in C_{0}^{\infty}\left(\mathbf{R}^{2}\right): \mathbf{R}^{2} \rightarrow \mathbf{R}$ are real smooth functions with compact support and that the total flux $\alpha=(2 \pi)^{-1} \int b(x) d x$ satisfies $0<\alpha<1$. Then the main theorem is formulated as follows.

THEOREM 7.1. Assume that the above assumptions are fulfilled. Let $f_{\alpha}\left(\omega^{\prime}-\omega\right)$ be as in Proposition 3.1 and let $\mathcal{E}_{1}$ denote the resonance space at zero energy of the Hamiltonian $H=H(A, V)$. Set $c(\lambda)=(2 \pi / i \sqrt{\lambda})^{1 / 2}$ again. Then the scattering amplitude $f\left(\omega \rightarrow \omega^{\prime} ; \lambda\right)$ for scattering from initial direction $\omega$ to final one $\omega^{\prime}$ at energy $\lambda$ obeys the following asymptotic formula as $\lambda \rightarrow 0$.

(1) If $\operatorname{dim} \mathcal{E}_{1}=2$, then

$$
f\left(\omega \rightarrow \omega^{\prime} ; \lambda\right)=c(\lambda)\left(f_{\alpha}\left(\omega^{\prime}-\omega\right)+(i / \pi) \sin \alpha \pi\left(1-e^{i\left(\omega^{\prime}-\omega\right)}\right)+o(1)\right) .
$$

(2) Assume that $\operatorname{dim} \mathcal{E}_{1}=1$. Let

$$
\rho=c_{0} r^{-\alpha}+c_{1} r^{-(1-\alpha)} e^{i \theta}+g, \quad g \in L^{2}\left(\mathbf{R}^{2}\right),
$$

be a resonance function spanning $\mathcal{E}_{1}$. Then one has:

(i) Assume that $0<\alpha<1 / 2$. If $c_{0} \neq 0$, then

$$
f\left(\omega \rightarrow \omega^{\prime} ; \lambda\right)=c(\lambda)\left(f_{\alpha}\left(\omega^{\prime}-\omega\right)+(i / \pi) \sin \alpha \pi+o(1)\right)
$$

and if $c_{0}=0$, then

$$
f\left(\omega \rightarrow \omega^{\prime} ; \lambda\right)=c(\lambda)\left(f_{\alpha}\left(\omega^{\prime}-\omega\right)-(i / \pi) \sin \alpha \pi e^{i\left(\omega^{\prime}-\omega\right)}+o(1)\right) .
$$


(ii) Assume that $\alpha=1 / 2$. Set $c=c_{1} / c_{0}\left(c=\infty\right.$ if $\left.c_{0}=0\right)$. Then

$$
f\left(\omega \rightarrow \omega^{\prime} ; \lambda\right)=c(\lambda)\left(f_{\alpha}\left(\omega^{\prime}-\omega\right)+\frac{i\left(1-\bar{c} e^{-i \omega}\right)\left(1+c e^{i \omega^{\prime}}\right)}{\pi\left(1+|c|^{2}\right)}+o(1)\right)
$$

(iii) Assume that $1 / 2<\alpha<1$. If $c_{1} \neq 0$, then

$$
f\left(\omega \rightarrow \omega^{\prime} ; \lambda\right)=c(\lambda)\left(f_{\alpha}\left(\omega^{\prime}-\omega\right)-(i / \pi) \sin \alpha \pi e^{i\left(\omega^{\prime}-\omega\right)}+o(1)\right)
$$

and if $c_{1}=0$, then

$$
f\left(\omega \rightarrow \omega^{\prime} ; \lambda\right)=c(\lambda)\left(f_{\alpha}\left(\omega^{\prime}-\omega\right)+(i / \pi) \sin \alpha \pi+o(1)\right) .
$$

(3) Assume that $\operatorname{dim} \mathcal{E}_{1}=0$. Then

$$
f\left(\omega \rightarrow \omega^{\prime} ; \lambda\right)=c(\lambda)\left(f_{\alpha}\left(\omega^{\prime}-\omega\right)+o(1)\right) .
$$

Remark. We make a brief comment on the case $\alpha \notin \mathbf{Z}$. If $\alpha \notin \mathbf{Z}$, then $(-1)^{[\alpha]} f\left(\omega \rightarrow \omega^{\prime} ; \lambda\right),[]$ being the Gauss notation, obeys the asymptotic formula in the theorem.

\section{$\S 8$. Scattering by magnetic field with small support}

As a simple application of Theorem 7.1, we here study the scattering by magnetic fields with small support. Let $H_{\varepsilon}$ be again defined by

$$
H_{\varepsilon}=H\left(A_{\varepsilon}, V_{\varepsilon}\right)=\left(-i \nabla-A_{\varepsilon}\right)^{2}+V_{\varepsilon}, \quad 0<\varepsilon \ll 1,
$$

where $V_{\varepsilon}(x)=\varepsilon^{-2} V(x / \varepsilon)$ and $A_{\varepsilon}(x)=\varepsilon^{-1} A(x / \varepsilon)$ with $\nabla \times A_{\varepsilon}=b_{\varepsilon}=$ $\varepsilon^{-2} b(x / \varepsilon)$. We denote by $f_{\varepsilon}\left(\omega \rightarrow \omega^{\prime} ; \lambda\right)$ the scattering amplitude for the pair $\left(H_{\varepsilon}, H_{0}\right)$. The next theorem is obtained as an immediate consequence of Corollary 3.1 and Theorem 7.1 .

THEOREM 8.1. Let the notations and assumptions be as in Theorem 7.1. Then the scattering amplitude $f_{\varepsilon}\left(\omega \rightarrow \omega^{\prime} ; \lambda\right), \lambda>0$ being fixed, obeys the following asymptotic formula as $\varepsilon \rightarrow 0$.

(1) If $\operatorname{dim} \mathcal{E}_{1}=2$, then

$$
f_{\varepsilon}\left(\omega \rightarrow \omega^{\prime} ; \lambda\right)=c(\lambda)\left(f_{\alpha}\left(\omega^{\prime}-\omega\right)+(i / \pi) \sin \alpha \pi\left(1-e^{i\left(\omega^{\prime}-\omega\right)}\right)\right)+o(1) .
$$

(2) Assume that $\operatorname{dim} \mathcal{E}_{1}=1$. Let

$$
\rho=c_{0} r^{-\alpha}+c_{1} r^{-(1-\alpha)} e^{i \theta}+g, \quad g \in L^{2}\left(\mathbf{R}^{2}\right)
$$


again denote a resonance function spanning $\mathcal{E}_{1}$. Then one has:

(i) Assume that $0<\alpha<1 / 2$. If $c_{0} \neq 0$, then

$$
f_{\varepsilon}\left(\omega \rightarrow \omega^{\prime} ; \lambda\right)=c(\lambda)\left(f_{\alpha}\left(\omega^{\prime}-\omega\right)+(i / \pi) \sin \alpha \pi\right)+o(1)
$$

and if $c_{0}=0$, then

$$
f_{\varepsilon}\left(\omega \rightarrow \omega^{\prime} ; \lambda\right)=c(\lambda)\left(f_{\alpha}\left(\omega^{\prime}-\omega\right)-(i / \pi) \sin \alpha \pi e^{i\left(\omega^{\prime}-\omega\right)}\right)+o(1) .
$$

(ii) Assume that $\alpha=1 / 2$. Set $c=c_{1} / c_{0}\left(c=\infty\right.$ if $\left.c_{0}=0\right)$. Then

$$
f_{\varepsilon}\left(\omega \rightarrow \omega^{\prime} ; \lambda\right)=c(\lambda)\left(f_{\alpha}\left(\omega^{\prime}-\omega\right)+\frac{i\left(1-\bar{c} e^{-i \omega}\right)\left(1+c e^{i \omega^{\prime}}\right)}{\pi\left(1+|c|^{2}\right)}\right)+o(1) .
$$

(iii) Assume that $1 / 2<\alpha<1$. If $c_{1} \neq 0$, then

$$
f_{\varepsilon}\left(\omega \rightarrow \omega^{\prime} ; \lambda\right)=c(\lambda)\left(f_{\alpha}\left(\omega^{\prime}-\omega\right)-(i / \pi) \sin \alpha \pi e^{i\left(\omega^{\prime}-\omega\right)}\right)+o(1)
$$

and if $c_{1}=0$, then

$$
f_{\varepsilon}\left(\omega \rightarrow \omega^{\prime} ; \lambda\right)=c(\lambda)\left(f_{\alpha}\left(\omega^{\prime}-\omega\right)+(i / \pi) \sin \alpha \pi\right)+o(1)
$$

(3) Assume that $\operatorname{dim} \mathcal{E}_{1}=0$. Then

$$
f_{\varepsilon}\left(\omega \rightarrow \omega^{\prime} ; \lambda\right)=c(\lambda) f_{\alpha}\left(\omega^{\prime}-\omega\right)+o(1)
$$

If, in particular, $\alpha=1 / 2$, then the theorem above shows the lack of conservation of angular momentum in the limit $\varepsilon \rightarrow 0$. For example, incoming particles with only $l=0$ as angular momentum may have angular momentum $l=1$ after scattering by the field $b_{\varepsilon}$ with support small enough.

When $\varepsilon \rightarrow 0, H_{\varepsilon}$ is formally convergent to the Hamiltonian

$$
H_{0 \alpha}=\left(-i \nabla-B_{\alpha}\right)^{2}, \quad \mathcal{D}\left(H_{0 \alpha}\right)=C_{0}^{\infty}\left(\mathbf{R}^{2} \backslash\{0\}\right)
$$

defined by (1.4). This has the $\delta$-like magnetic field $\nabla \times B_{\alpha}=2 \pi \alpha \delta(x)$ at the orgin. We discuss the relation between the limits in the above theorem and the scattering amplitude for the Hamiltonian obtained as a self-adjoint extension of $H_{0 \alpha}$. 
We denote by $\bar{H}_{0 \alpha}$ the closure of $H_{0 \alpha}$. This is symmetric, but is not self-adjoint. Let $\Sigma_{ \pm}=\operatorname{Ker}\left(\bar{H}_{0 \alpha}^{*} \mp i\right)$. Then $\Sigma_{ \pm}$is spanned by $\psi_{ \pm l}, l=0,1$, where

$$
\psi_{+l}(x)=\tau_{l} K_{\nu}\left(e^{-i \pi / 4} r\right) e^{i l \theta}, \quad \psi_{-l}(x)=\tau_{l} e^{i \nu \pi / 2} K_{\nu}\left(e^{i \pi / 4} r\right) e^{i l \theta}
$$

with the modified Bessel function $K_{\nu}(z)=(i \pi / 2) e^{i \nu \pi / 2} H_{\nu}(i z)$. The constant $\tau_{l}>0$ is determined by normalization $\left\|\psi_{ \pm l}\right\|_{L^{2}\left(\mathbf{R}^{2}\right)}=1$, and the phase factor $e^{i \nu \pi / 2}$ is taken so that $\psi_{+l}-\psi_{-l} \rightarrow 0$ as $r \rightarrow 0$. Thus the closure $\bar{H}_{0 \alpha}$ has its deficiency indices $(2,2)$. By the general theory due to Krein, $\bar{H}_{0 \alpha}$ has a family of self-adjoint extensions parameterized by $2 \times 2$ unitary mapping from one deficiency space to the other one. Let

$$
U=U(\eta, a, b)=e^{i \eta}\left(\begin{array}{cc}
a & -\bar{b} \\
b & \bar{a}
\end{array}\right), \quad|a|^{2}+|b|^{2}=1, \quad \eta \in \mathbf{R}, \quad a, b \in \mathbf{C},
$$

be a $2 \times 2$ unitary matrix. We denote by the same notation $U$ the mapping $U: \Sigma_{+} \rightarrow \Sigma_{-}$defined by

$$
U \psi_{+}=\tilde{e}_{0} \psi_{-0}+\tilde{e}_{1} \psi_{-1}, \quad \psi_{+}=e_{0} \psi_{+0}+e_{1} \psi_{+1},
$$

with ${ }^{t}\left(\tilde{e}_{0}, \tilde{e}_{1}\right)=U^{t}\left(e_{0}, e_{1}\right)$. Then, for given $U=U(\eta, a, b)$, there exists a self-adjoint extension $H_{\alpha}^{U}$ such that

$$
H_{\alpha}^{U} u=\bar{H}_{0 \alpha} v+i \psi_{+}-i U \psi_{+}
$$

with domain

$$
\mathcal{D}\left(H_{\alpha}^{U}\right)=\left\{u \in L^{2}\left(\mathbf{R}^{2}\right): u=v+\psi_{+}+U \psi_{+}, v \in \mathcal{D}\left(\bar{H}_{0 \alpha}\right), \psi_{+} \in \Sigma_{+}\right\}
$$

The unitary matrix $U(\eta, a, b)$ specifies the boundary condition at the origin. If, for example, $U=U(0,-1,0)$, then the domain $\mathcal{D}\left(H_{\alpha}^{U}\right)$ is given by

$$
\mathcal{D}\left(H_{\alpha}^{U}\right)=\left\{u \in L^{2}\left(\mathbf{R}^{2}\right): H_{0 \alpha} u \in L^{2}\left(\mathbf{R}^{2}\right), \lim _{r \rightarrow 0} u(x)=0\right\}
$$

and this extension coincides with $H_{\alpha}$ defined by (1.3). We denote by $f^{U}(\omega \rightarrow$ $\left.w^{\prime} ; \lambda\right)$ the scattering amplitude for the pair $\left(H_{\alpha}^{U}, H_{0}\right)$. It is defined through the asymptotic behavior

$$
u(x)=e^{i \sqrt{\lambda} x \cdot \omega}+f^{U}\left(\omega \rightarrow w^{\prime} ; \lambda\right) e^{i \sqrt{\lambda} r} r^{-1 / 2}(1+o(1)), \quad x=r \omega^{\prime}, \quad|x| \rightarrow \infty
$$


of the solution $u(x)$ to equation $\left(H_{0 \alpha}-\lambda\right) u=0$, where $u(x)$ satisfies the boundary condition specified by $U(\eta, a, b)$ at the origin. The scattering amplitude $f^{U}\left(\omega \rightarrow w^{\prime} ; \lambda\right)$ has been calculated in the recent work [2] and it takes a rather complicated form. We do not copy the explicit form obtained there. If, in particular, $U=U(0,-1,0)$, then $f^{U}\left(\omega \rightarrow \omega^{\prime} ; \lambda\right)=c(\lambda) f_{\alpha}\left(\omega^{\prime}-\omega\right)$. As previously stated, this is just the scattering amplitude calculated by [1] (see [11] also). We mention only the results. According to the results in [2], Theorem 8.1 is now reformulated as follows.

THEOREM 8.2. Let the notations and assumptions be again as in Theorem 7.1.

(1) If $\operatorname{dim} \mathcal{E}_{1}=2$, then

$$
\lim _{\varepsilon \rightarrow 0} f_{\varepsilon}\left(\omega \rightarrow \omega^{\prime} ; \lambda\right)=f^{U}\left(\omega \rightarrow \omega^{\prime} ; \lambda\right)
$$

with $U=U(\pi / 2, \exp (i(1 / 2-\alpha) \pi), 0)$.

(2) Assume that $\operatorname{dim} \mathcal{E}_{1}=1$. Then one has:

(i) Assume that $0<\alpha<1 / 2$. If $c_{0} \neq 0$, then

$$
\lim _{\varepsilon \rightarrow 0} f_{\varepsilon}\left(\omega \rightarrow \omega^{\prime} ; \lambda\right)=f^{U}\left(\omega \rightarrow \omega^{\prime} ; \lambda\right)
$$

with $U=U((1-\alpha / 2) \pi, \exp (-i \alpha \pi / 2), 0)$, and if $c_{0}=0$, then

$$
\lim _{\varepsilon \rightarrow 0} f_{\varepsilon}\left(\omega \rightarrow \omega^{\prime} ; \lambda\right)=f^{U}\left(\omega \rightarrow \omega^{\prime} ; \lambda\right)
$$

with $U=U((1 / 2+\alpha / 2) \pi, \exp (i(1-\alpha) \pi / 2), 0)$.

(ii) Assume that $\alpha=1 / 2$ and set $c=c_{1} / c_{0}$ again. Then

$$
\lim _{\varepsilon \rightarrow 0} f_{\varepsilon}\left(\omega \rightarrow \omega^{\prime} ; \lambda\right)=f^{U}\left(\omega \rightarrow \omega^{\prime} ; \lambda\right)
$$

with $U=U(3 \pi / 4, a, b)$, where

$$
a=\frac{1}{\sqrt{2}}\left(1-i \frac{1-|c|^{2}}{1+|c|^{2}}\right), \quad b=\frac{2 i c}{\sqrt{2}\left(1+|c|^{2}\right)} .
$$

(iii) Assume that $1 / 2<\alpha<1$. If $c_{1} \neq 0$, then

$$
\lim _{\varepsilon \rightarrow 0} f_{\varepsilon}\left(\omega \rightarrow \omega^{\prime} ; \lambda\right)=f^{U}\left(\omega \rightarrow \omega^{\prime} ; \lambda\right)
$$

with $U=U((1 / 2+\alpha / 2) \pi, \exp (i(1-\alpha) \pi / 2), 0)$, and if $c_{1}=0$, then

$$
\lim _{\varepsilon \rightarrow 0} f_{\varepsilon}\left(\omega \rightarrow \omega^{\prime} ; \lambda\right)=f^{U}\left(\omega \rightarrow \omega^{\prime} ; \lambda\right)
$$


with $U=U((1-\alpha / 2) \pi, \exp (-i \alpha \pi / 2), 0)$.

(3) Assume that $\operatorname{dim} \mathcal{E}_{1}=0$. Then

$$
\lim _{\varepsilon \rightarrow 0} f_{\varepsilon}\left(\omega \rightarrow \omega^{\prime} ; \lambda\right)=f^{U}\left(\omega \rightarrow \omega^{\prime} ; \lambda\right)
$$

with $U=U(0,-1,0)$.

\section{$\S$ 9. Proof of Proposition 4.1}

In this section we prove Proposition 4.1 and Lemma 4.4, which remain unproved.

Proof of Proposition 4.1. As stated in Remark (3) after the proposition, (2) has been already verified. We prove (1) and (3) for the + case only. The - case follows by adjoint at once. The proof is rater long and it is divided into several steps.

(i) Let $R_{l}(r, p ; k)$ be the integral kernel of $R\left(k^{2}+i 0 ; X_{l}\right), k>0$. The kernel is given by

$$
R_{l}(r, p ; k)=-f_{l}(r \wedge p ; k) g_{l}(r \vee p ; k) / W\left(f_{l}, g_{l}\right),
$$

where

$$
\begin{aligned}
& f_{l}(r ; k)= \begin{cases}r^{1 / 2} J_{l}(k r) & 0<r \leq 1 \\
c_{1 l}(k) r^{1 / 2} J_{\nu}(k r)+c_{2 l}(k) r^{1 / 2} J_{-\nu}(k r) & r>1,\end{cases} \\
& g_{l}(r ; k)= \begin{cases}d_{1 l}(k) r^{1 / 2} J_{l}(k r)+d_{2 l}(k) r^{1 / 2} N_{l}(k r) & 0<r \leq 1 \\
r^{1 / 2} H_{\nu}(k r) & r>1,\end{cases}
\end{aligned}
$$

$N_{l}(z)$ being the Neumann function, and the four coefficients are determined so as to satisfy the connecting conditions at $r=1$. Since $W\left(J_{l}, N_{l}\right)(z)=$ $2 / \pi z$ and $W\left(J_{\nu}, J_{-\nu}\right)(z)=-2 \sin \nu \pi / \pi z$, the Wronskian $W\left(f_{l}, g_{l}\right)$ is calculated as

$$
W\left(f_{l}, g_{l}\right)=d_{2 l}(k) k W\left(J_{l}, N_{l}\right)(k)=2 d_{2 l}(k) / \pi
$$

and the four coefficients above are determined as follows:

$$
\begin{aligned}
& c_{1 l}(k)=(\pi / 2 \sin \nu \pi) k\left(J_{-\nu}(k) J_{l}^{\prime}(k)-J_{-\nu}^{\prime}(k) J_{l}(k)\right), \\
& c_{2 l}(k)=(\pi / 2 \sin \nu \pi) k\left(J_{l}(k) J_{\nu}^{\prime}(k)-J_{l}^{\prime}(k) J_{\nu}(k)\right), \\
& d_{1 l}(k)=(\pi / 2) k\left(H_{\nu}(k) N_{l}^{\prime}(k)-H_{\nu}^{\prime}(k) N_{l}(k)\right), \\
& d_{2 l}(k)=(\pi / 2) k\left(J_{l}(k) H_{\nu}^{\prime}(k)-J_{l}^{\prime}(k) H_{\nu}(k)\right) .
\end{aligned}
$$


Hence we have

$$
R_{l}(r, p ; k)=f_{l}(r \wedge p ; k) h_{l}(r \vee p ; k)
$$

where

$$
h_{l}(r ; k)=e_{l}(k) g_{l}(k ; r), \quad e_{l}(k)=-\pi / 2 d_{2 l}(k) .
$$

(ii) We investigate only the case $l=0$ or 1 in some detail. We first look at the behavior as $k \rightarrow 0$ of $f_{l}(r ; k)$. Recall the representation for the pair $\left(u_{l}, v_{l}\right), l=0,1$, of linearly independent solutions to $X_{l} u=0$. If $0<r \leq 1$, then

$$
f_{l}(r ; k)=(l+\nu)^{1 / 2}(k / 2)^{l}\left(u_{l}(r)+O\left(k^{2}\right)\right) .
$$

By the asymptotic formula of Bessel functions, we have

$$
\begin{aligned}
& c_{1 l}(k)=(\pi / 2 \sin \nu \pi)((l+\nu) / \Gamma(1-\nu))(k / 2)^{l-\nu}\left(1+O\left(k^{2}\right)\right), \\
& c_{2 l}(k)=-(\pi / 2 \sin \nu \pi)((l-\nu) / \Gamma(1+\nu))(k / 2)^{l+\nu}\left(1+O\left(k^{2}\right)\right) .
\end{aligned}
$$

Since $\Gamma(1+\nu) \Gamma(1-\nu)=\nu \pi / \sin \nu \pi$, this implies that $f_{l}(r ; k)$ still obeys the same relation as above for $r>1$ and hence we obtain

$$
f_{l}(r ; k)=(l+\nu)^{1 / 2}(k / 2)^{l}\left(u_{l}(r)+O\left(k^{2}\right)\right)
$$

locally uniformly in $r \geq 0$.

(iii) Let $e_{l}(k)$ be as above. We study the behavior of $e_{l}(k)$. Recall that $\beta_{l}(k)$ in the proposition is defined by

$$
\beta_{l}(k)=-((l-\nu) \Gamma(1-\nu) /(l+\nu) \Gamma(1+\nu)) e^{-i \nu \pi}(k / 2)^{2 \nu}
$$

and hence $\beta_{l}(k)=\left(c_{2 l}(k) / c_{1 l}(k)\right) e^{-i \nu \pi}\left(1+O\left(k^{2}\right)\right)$. Since $H_{\nu}(z)$ is represented by the formula

$$
H_{\nu}(z)=(i / \sin \nu \pi)\left(J_{\nu}(z) e^{-i \nu \pi}-J_{-\nu}(z)\right)
$$

we have $d_{2 l}(k)=i\left(c_{2 l}(k) e^{-i \nu \pi}+c_{1 l}(k)\right)$, so that

$$
d_{2 l}(k)=i c_{1 l}(k)\left(1+\beta_{l}(k)\right)\left(1+O\left(k^{2}\right)\right)
$$

Thus $e_{l}(k), l=0,1$, behaves like

$$
(9.5) e_{l}(k)=i \sin \nu \pi(\Gamma(1-\nu) /(l+\nu))\left(1+\beta_{l}(k)\right)^{-1}(k / 2)^{\nu-l}\left(1+O\left(k^{2}\right)\right) .
$$


(iv) We analyse the behavior as $k \rightarrow 0$ of $h_{l}(r ; k)$ in (9.1). According to (9.4), we decompose it into the sum of two terms $h_{l}=I_{+}(r ; k)-I_{-}(r ; k)$ for $r>1$, where

$$
\begin{aligned}
& I_{+}(r ; k)=(i / \sin \nu \pi) e^{-i \nu \pi} e_{l}(k) J_{\nu}(k r) r^{1 / 2} \\
& I_{-}(r ; k)=(i / \sin \nu \pi) e_{l}(k) J_{-\nu}(k r) r^{1 / 2} .
\end{aligned}
$$

Since $\gamma_{l}(k)=2 \nu(l-\nu)^{-1} \beta_{l}(k)\left(1+\beta_{l}(k)\right)^{-1}$, it follows from (9.5) that

$$
\begin{aligned}
I_{-}(r ; k) & =-(l+\nu)^{-1}\left(1+\beta_{l}(k)\right)^{-1}(k / 2)^{-l}\left(r^{-\nu+1 / 2}+O\left(k^{2}\right)\right) \\
& =-(l+\nu)^{-1}\left(1-\beta_{l}(k)\left(1+\beta_{l}(k)\right)^{-1}\right)(k / 2)^{-l}\left(r^{-\nu+1 / 2}+O\left(k^{2}\right)\right) \\
& =-(l+\nu)^{-1}(k / 2)^{-l}\left(\left(1+(1 / 2-l / 2 \nu) \gamma_{l}(k)\right) r^{-\nu+1 / 2}+O\left(k^{2}\right)\right)
\end{aligned}
$$

locally uniformly in $r \geq 1$. If we use (9.3), we obtain in a similar way that

$$
\begin{aligned}
I_{+}(r ; k) & =(l-\nu)^{-1} \beta_{l}(k)\left(1+\beta_{l}(k)\right)^{-1}(k / 2)^{-l}\left(r^{\nu+1 / 2}+O\left(k^{2}\right)\right) \\
& =(l+\nu)^{-1}(k / 2)^{-l}\left((1 / 2+l / 2 \nu) \gamma_{l}(k) r^{\nu+1 / 2}+O\left(k^{2}\right)\right) .
\end{aligned}
$$

Hence $h_{l}(r ; k)$ behaves like

$$
h_{l}(r ; k)=(l+\nu)^{-1 / 2}(k / 2)^{-l}\left(v_{l}(r)+\gamma_{l}(k) u_{l}(r)+O\left(k^{2}\right)\right)
$$

for $r>1$. This, together with (9.1) and (9.2), implies that

$$
R_{l}(r, p ; k)=u_{l}(r \wedge p) v_{l}(r \vee p)+\gamma_{l}(k) u_{l}(r) u_{l}(p)+O\left(k^{2}\right)
$$

locally uniformly in $(r, p) \in[0, \infty) \times[0, \infty)$, when $r \vee p \geq 1$.

(v) We represent $h_{l}(r ; k)$ as

$$
h_{l}(r ; k)=d_{1 l}(k) e_{l}(k) J_{l}(k r) r^{1 / 2}-(\pi / 2) N_{l}(k r) r^{1 / 2}
$$

for $0<r \leq 1$ and we continue to analyse its behavior as $k \rightarrow 0$. We look at the behavior of $d_{1 l}(k) e_{l}(k)$. For brevity, we consider only the case $l=0$. The Neumann function $N_{0}(z)$ behaves like

$$
(\pi / 2) N_{0}(z)=(\gamma+\log (z / 2)) J_{0}(z)+O\left(|z|^{2}\right), \quad|z| \rightarrow 0,
$$

where $\gamma$ is the Euler constant. Hence $d_{10}(k)$ obeys

$$
d_{10}(k)=-(2 / \pi)(\gamma+\log (k / 2)) d_{20}(k)+H_{\nu}(k) J_{0}(k)+O\left(k^{-\nu+2}\right) .
$$


By (9.4), we further see that

$$
d_{10}(k)=(\gamma+\log (k / 2)) / e_{0}(k)+d(k)+O\left(k^{-\nu+2}\right),
$$

where

$$
d(k)=\frac{i}{\sin \nu \pi}\left(\frac{1}{\Gamma(1+\nu)}(k / 2)^{\nu} e^{-i \nu \pi}-\frac{1}{\Gamma(1-\nu)}(k / 2)^{-\nu}\right) .
$$

If we make use of (9.3) and (9.5) with $l=0$, then

$$
\begin{aligned}
d(k) e_{0}(k) & =\left(1 / \nu-\beta_{0}(k) / \nu\right)\left(1+\beta_{0}(k)\right)^{-1}+O\left(k^{2}\right) \\
& =1 / \nu+\gamma_{0}(k) / \nu+O\left(k^{2}\right)
\end{aligned}
$$

and hence we have

$$
d_{10}(k) e_{0}(k)=\left((\gamma+\log (k / 2))+1 / \nu+\gamma_{0}(k) / \nu\right)+O\left(k^{2}\right) .
$$

This, together with (9.7), yields that

$$
\begin{aligned}
h_{0}(r ; k) & =\left((1 / \nu-\log r)+\left(\gamma_{0}(k) / \nu\right)\right) r^{1 / 2} J_{0}(k r)+O\left(k^{2}\right) \\
& =\nu^{-1 / 2}\left(v_{0}(r)+\gamma_{0}(k) u_{0}(r)\right)+O\left(k^{2}\right)
\end{aligned}
$$

uniformly in $0 \leq r \leq 1$. Hence it follows from (9.2) and (9.6) that

$$
R_{0}(r, p ; k)=u_{0}(r \wedge p) v_{0}(r \vee p)+\gamma_{0}(k) u_{0}(r) u_{0}(p)+O\left(k^{2}\right)
$$

locally uniformly in $(r, p) \in[0, \infty) \times[0, \infty)$. If we check the argument through the above steps more carefully, we see that

$$
R_{0}(r, p ; k)=u_{0}(r \wedge p) v_{0}(r \vee p)+\gamma_{0}(k) u_{0}(r) u_{0}(p)+k^{2} E_{02}(r, p)+o\left(k^{2}\right)
$$

for some function $E_{02}(r, p)$ bounded locally. Thus (1) is proved for $l=0$ and (3) is immediately obtained. A similar argument applies to the case $l=1$. We skip the details. The case $l \neq 0,1$ is much easier to prove. If $l \neq 0,1$, then $\nu>1$ and $R_{l}(r, p ; k)$ is shown to be expanded as

$$
R_{l}(r, p ; k)=u_{l}(r \wedge p) v_{l}(r \vee p)+k^{2} E_{l 2}(r, p)+o\left(k^{2}\right)
$$

locally uniformly in $(r, p)$. The proof of the proposition is now completed. 
We end the paper by proving Lemma 4.4.

Proof of Lemma 4.4. As is seen from the proof of Proposition 4.1,

$$
\left(R_{l}\left(k^{2}+i 0 ; X_{l}\right) f\right)(r)=e_{l}(k) r^{1 / 2} H_{\nu}(k r) \int_{0}^{r} f_{l}(p ; k) f(p) d p, \quad r \gg 1,
$$

for $f \in L_{\text {com }}^{2}\left(I_{L}\right)$. Since $f_{l}(r ; k)=O\left(k^{l}\right)$ locally uniformly in $r \geq 0$ and since $e_{l}(k)$ obeys $e_{l}(k)=O\left(k^{\nu-l}\right)$, it follows that

$$
\left(R_{l}\left(k^{2}+i 0 ; X_{l}\right) f\right)(r)=O\left(k^{\nu}\right) r^{1 / 2} H_{\nu}(k r), \quad k \rightarrow 0,
$$

uniformly in $r \gg 1$. This remains true for $\zeta$ with $\operatorname{Im} \sqrt{\zeta} \geq 0$. Thus the lemma is proved.

\section{REFERENCES}

[1] Y. Aharonov and D. Bohm, Significance of electromagnetic potential in the quantum theory, Phys. Rev., 115 (1959), 485-491.

[2] R. Adami and A. Teta, On the Aharonov-Bohm Hamiltonian, Lett. Math. Phys., 43 (1998), 43-53.

[3] S. Albeverio, F. Gesztesy, R. Hoegh-Krohn and H. Holden, Solvable Models in Quantum Mechanics, Springer-Verlag, 1988.

[4] D. Bollé, F. Gesztesy and C. Danneels, Threshold scattering in two dimensions, Ann. Inst. Henri Poincaré, 48 (1988), 175-204.

[5] A. Erdélyi, Higher Transcendental Functions Vol II, Robert E. Krieger Publishing Company, 1953.

[6] T. Ikebe and Y. Saitō, Limiting absorption method and absolute continuity for the Schrödinger operators, J. Math. Kyoto Univ., 7 (1972), 513-542.

[7] A. Jensen and T. Kato, Spectral properties of Schrödinger operators and time - decay of the wave functions, Duke Math. J., 46 (1979), 583-611.

[8] M. Loss and B. Thaller, Scattering of particles by long-range magnetic fields, Ann. of Phys., 176 (1987), 159-180.

[9] M. Murata, Asymptotic expansions in time for solutions of Schrödinger-type equations, J. Func. Anal., 49 (1982), 10-56.

[10] P. A. Perry, Scattering Theory by the Enss Method, Mathematical Reports 1, Harwood Academic, 1983.

[11] S. N. M. Ruijsenaars, The Ahanorov-Bohm effect and scattering theory, Ann. of Phys., 146 (1983), 1-34.

[12] S. Shimada, Low energy scattering with a penetrable wall interaction, J. Math. Kyoto Univ., 34 (1994), 95-147.

[13] H. Tamura, Semi-classical analysis for total cross-sections of magnetic Schrödinger operators in two dimensions, Rev. Math. Phys., 7 (1995), 443-480. 
[14] L. Dabrowski and P. Stovicek, Aharonov-Bohm effect with $\delta$-type interaction, J. Math. Phys., 39 (1998), 47-62.

\author{
Department of Mathematics \\ Okayama University \\ Okayama 700-8530 \\ Japan \\ tamura@math.okayama-u.ac.jp
}

